\title{
Data Acquisition System for Light-Ion Irradiation Creep Experiment
}

P. L. Hendrick

T. J. Whitaker

July 1979

Prepared for the U.S. Department of Energy under Contract EY-76-C-06-1830

Pacific Northwest Laboratory Operated for the U.S. Department of Energy by Battelle Memorial Institute 


\title{
NOTICE
}

This report was prepared as an account of work sponsored by the United States Government. Neither the United States nor the Department of Energy, nor any of their employees, nor any of their contractors, subcontractors, or their employees, makes any warranty, express or implied, or assumes any legal liability or responsibility for the accuracy, completeness or usefulness of any information, apparatus, product or process disclosed, or represents that its use would not infringe privately owned rights.

The views, opinions and conclusions contained in this report are those of the contractor and do not necessarily represent those of the United States Government or the United States Department of Energy.

\author{
PACIFIC NORTHWEST LABORATORY \\ operated by \\ BATTELLE \\ for the \\ UNITED STATES DEPARTMENT OF ENERGY \\ Under Contract EY-76-C-06-1830
}
Printed in the United States of America Available from
National Technical Information Service
United States Department of Commerce
5285 Port Royal Road
Springfield, Virginia 22151

Price: Printed Copy \$

$\therefore$ Microfiche $\$ 3.00$

NTIS

-Pages Selling Price

001-025 $\quad \$ 4.00$

026-050 $\quad 54.50$

$051-075 \quad \$ 5.25$

076-100 $\quad 56.00$

$101-125 \quad \$ 6.50$

$126.150 \quad \$ 7.25$

$151-175 \quad 58.00$

$176-200 \quad 59.00$

201-225 $\$ 9.25$

$226-250 \quad 59.50$

251-275 $\quad 510.75$

$276.300 \quad \$ 11.00$ 


\section{2}

\section{DATA ACQUISITION SYSTEM FOR \\ LIGHT-ION IRRADIATION CREEP EXPERIMENT}

P. L. Hendrick

T. J. Whitaker

July 1979

Prepared for the

U.S. Department of Energy

under Contract EY-76-C-06-?830

Pacific Northwest Labcratory

Operated for the U.S. Department of Energy

by Battelle Memorial Institute 


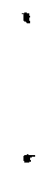

$\checkmark$

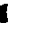




\section{ACKNOWLEDGEMENTS}

The authors acknowledge the support provided by the Division of Basic Energy Sciences, U.S. Department of Energy. Additionally, the authors acknowledge hardware support provided by Jan Goolsbey and Jerry Jackman of the Craft and Operation Services Department and comments received from Conny Pavloff and Charles Henager, ur. during the software development period. Finally, the editorial review provided by undy Hooper is gratefully acknowledged. 
,

. 


\section{CONTENTS}

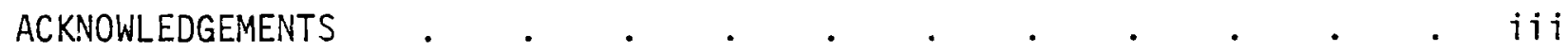

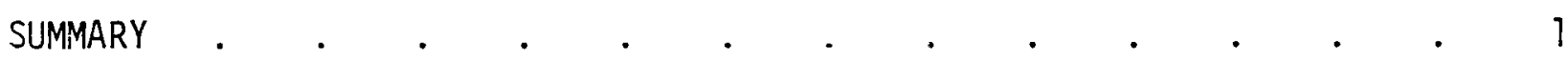

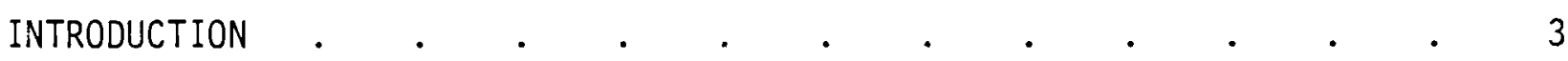

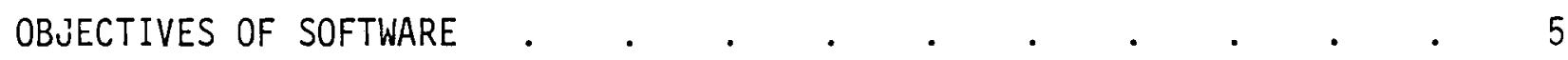

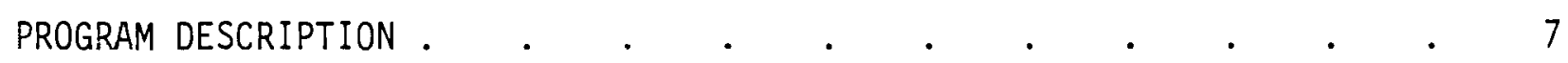

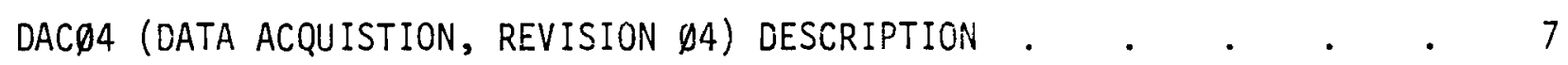

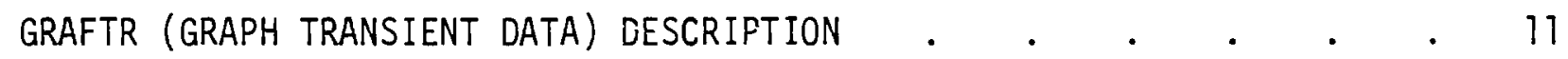

GRAFAV (GRAPH AVERAGED DATA) DESCRIPTION . . . . . . . . . . . 13

TYPAV (TYPE AVERAGED DATA) DESCRIPTION .

TYPLOG (TYPE LOG) DESCRIPTION • . . . . . . . . . . . . . . . 16

RETXT (READ TEST DATA) DESCRIPTION .

REINT3 (IREAD INITIALIZATION DATA, REVISION 3) DESCRIPTION • • . 17

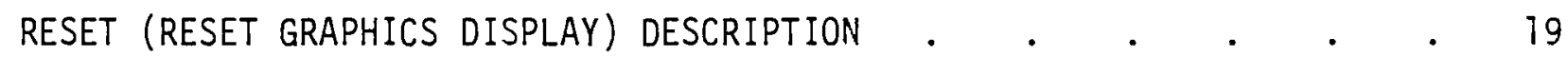

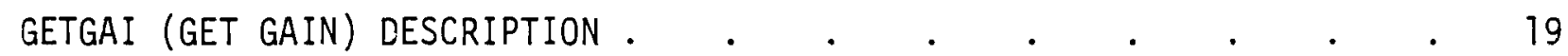

TIMDAT (GET TIME AND DATE) DESCRIPTION .

DECDAT (DECODE DATE) DESCRIPTION

ULIB (UTILITY LIBRARY) DESCRIPTION .

ULIBI (UTILITY LIBRARY, REVISION 1) DESCRIPTION . . . . . . . 23

PROGRAM OPERATION

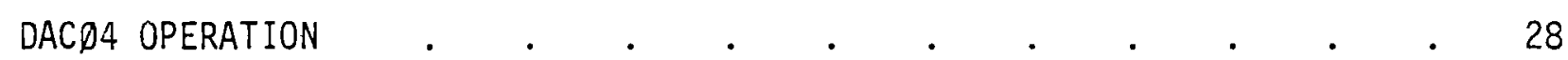

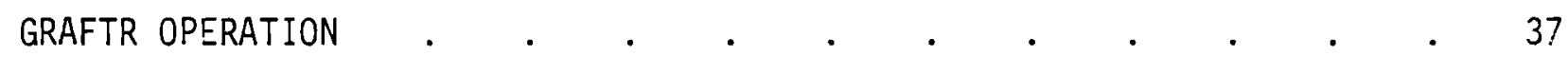

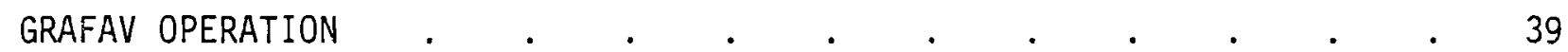

TYPAV OPERATION

TYPLOG OPERATION

RETXT OPERATION

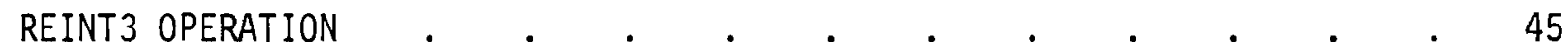

RESET OPERATION

APPENDIX A (COMPUTER HARDWARE DESCRIPTION) . . . . . . . . A-1

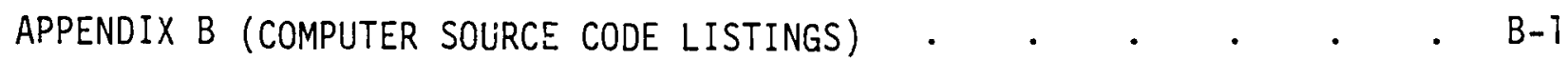

APPENDIX C (PROCEDURE FOR MAKING DELETED -FILES PERMANENT) • • • • c c-1

APPENDIX D (HARDWARE ADDRESS AND VECTOR LOCATIONS). . . . . . D-1

APPENDIX E (SPECIAL TIMING CONSIDERATIONS FOR DACZ4) . . • • • E E-1

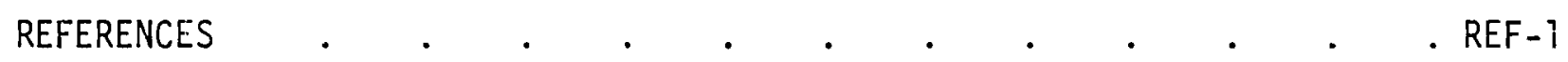




\section{FIGURES}

1 Example of Graph Produced by GRAFTR $\quad . \quad$. $\quad . \quad$ • $\quad . \quad 12$

2 Example of Graph Produced by GRAFAV . . . . . . . . 15

\section{$\underline{\text { TABLES }}$}

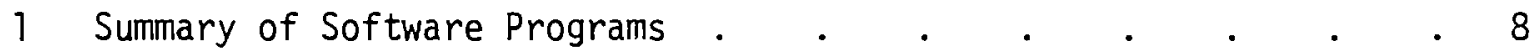

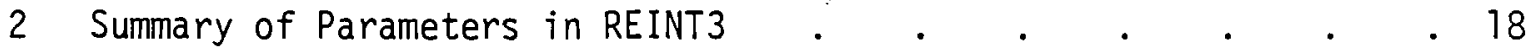

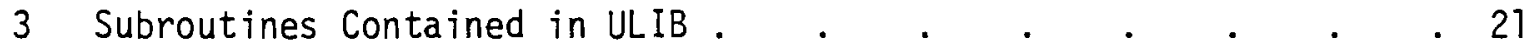

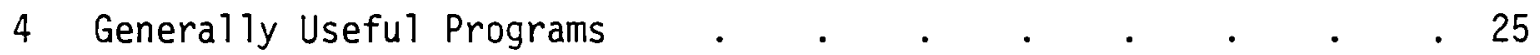

5 MACRO and FORTRAN Development Programs . • • • • . . . . 26

6 Summary of Disk Space Utilization . . . . . . . . . . . . 31

7 Gain Code Interpretation . . . . . . . . . . . . . . 31

8 DAC $\emptyset 4$ Input Codes . . . . . . . . . . . . . . . 36

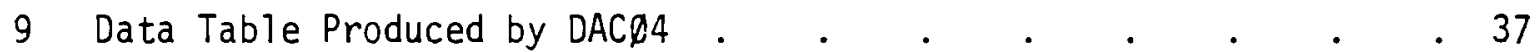

10 Disk Block Data Produced by TYPAV . . . . . . . . . 41

11 Two Channel Data Produced by TYPAV . . . . . . . . . . 43

12 Initialization Data Produced by REINT3 . . . . . . . . . 46 


\section{DATA ACQUISITION SYSTEM FOR \\ LIGHT-ION IRRADIATION CREEP EXPERIMENT}

\section{SUMMARY}

Software has been developed for a PDP17V/03-based data acquisition system to support the Light-Ion Irradiation Creep Experiment installed at the University of Washington Tandem Van de Graaff Accelerator. The software consists of a realtime data acquisition and storage program, DACQ4, written in assembly language. This program provides for the acquisition of up to 30 channels at $100 \mathrm{~Hz}$, data averaging before storage on disk, alarming, data table display, and automatic disk switching. All analog data are acquired via an amplifier-per-channel type analog-to-digital converter subsystem having a resolution of 14 bits, a maximum throughput of $20 \mathrm{kHz}$, and an overa 11 system accuracy of $\pm 0.01 \%$. These specifications are considered essential for the long-term measurement of irradiation creep strains and temperatures during the light-ion bombardment of irradiation creep specimens.

The software package developed also contains a collection of FORTRAN programs designed to monitor a test while in progress. These programs use the foreground/background feature of the RT-11 operating system. Under this system, the real-time program runs in foreground and has a high priority. Whenever the foreground job is suspended, a program operating in background is free to run until the foreground program requires service. In this manner, the user may monitor the test without interrupting the periodic acquisition of data.

The background programs provide a variety of services. The program, GRAFTR, allows transient data (i.e., prior to averaging) to be graphed at the graphics terminal. This program can be used as a diagnostic tool to assure, for example, that each channel is free of noise. The program, GRAFAV, allows averaged data to be read from disk and displayed graphically at the terminal. The user determines the time scale of the graphed data through his choice of the clock interrupt period and number of words averaged. The program, TYPAV, reads averaged data from disk and displays it at the terminal in tabular form. 
Other programs allow text messages to be written to disk, read from disk, and allow access to DACØ4 initialization data.

Although the software was designed to provide a stand-alone package, which could be operated by anyone familiar with RT-11, it is expected that the described programs will continue to evolve as actual operating experience is acquired. To this end, software has been developed in as straightforward a manner as possible to facilitate future modifications and/or enhancements. For example, extensive use has been made of DEC-supplied subroutines that permit simple modification and provide a large degree of flexibility. 


\section{INTRODUCTION}

This document describes software developed to support the Light-Ion Irradiation Creep Experiment presently insta?led at the University of Washington Tandem Van de Graafi Accelerator. This software was developed on and for use with a Digital Equipment Corporation (DEC) PDP11V/03 computer equipped with a Neff Model $620 / 100$ analog-to-digital converter (ADC). The computer is configured with $32 K$ words of memory, 16 channels of digital input, 32 channels of digital output, 4 channels of digital-to-analog output, 24 channels of analog-to-digital input, a dual floppy disk, a DEC VT-55 graphics terminal with hardcopy, 4 channels of seriāi interface, and a real time programmable clock.

The PDPIIV/03 computer was purchased with the DEC RT-11 operating system and documentation. The RT-11 operating system provides a dual-job operating environment in which high-priority jobs are run in foreground and low-priority jobs are run in background. In the current appiication, the real-time acquisition of data is accomplished in foreground. Graphic display and other lower priority functions are performed in background. In this way, the real-time data acquisition is always given priority and background jobs are only serviced while the foreground job is idle (suspended). The real-time programming is written in MACRO (DEC's assembly language software) to speed execution. However, most background programming is written in FORTRAN. BASIC is also included but has not yet been utilized. In severai instances, the FORTRAN programs call MACRO subroutines (e.g., in cases where bit manipulation is required).

We assume in this report that the user is familiar with the use of RT-11. For example, the PIF routina provides the capability of copying files from one disk to another and the EDIT routine allows one to modify and/or create code. These operations and others are described in a series of four volumes supplied with RT-11.

This report describes the objectives of the software and then the various software programs. This is followed by a discussion of program operations. Appendices include a description of the hardware, listings of the source codes, the procedure for making detailed files permanent, hardware address and vector locations, and special timing considoration for the real time program, DACD4. 
(

-

$\checkmark$

,

. 


\section{OBJECTIVES OF SOFTWARE}

The general objectives of the software were specified at the onset of development as follows:

- Provide the real-time acquisition of up to 24 analog input signals at a rate such that the sample period is small compared to the specimen time constant $\left(\tau \sim 200 \mathrm{msec}\right.$ at $\left.200^{\circ} \mathrm{C}\right)$.

- Average the data prior to storage on disk to more efficiently use disk space and increase the period between writes to disk.

- Store channel number, programmable gain, date and time in addition to data on each disk block to facilitate post-test analysis.

- Provide an auto-ranging option (via programmable gain).

- Provide the capability to display in graphical form both transient (i.e., prior to averaging) and averaged data.

- Provide the capability to restart the real-time data acquisition program within 1 min if a computer crash occurs.

- Provide the capability of automatically switching disks when a disk is full.

- Provide alarm capability should selected channels exceed preset levels. Based upon comments and evaluation during software development, several enhancements have been added:

- The foreground program has the option of providing an automatically updated table of current data in millivolts, also showing fixed and programmable gain.

- The foreground program automatically writes a text block that allows the user to record comments relative to a particular experiment.

- A background program allows the user to write text data to disk during the test if he wishes to record comments concerning the test conduct. 
- The foreground program automatically records on a disk block appropriate initialization data that may be helpful during post-test data analysis and which enables an automatic restart of DAC 04 if desired.

- Background programs allow the user to read text data or initialization data.

- The user may alter, on-line, the number of data words averaged.

Other enhancements are conceptually feasible, but will not be implemented until after the computer is installed at the University and the software is evaluated. These potential enhancements include the following:

- Control specimen temperature with one of four digital-to-analog output channels. Implementation of this enhancement must await an evaluation of the computer's reliability (e.g., a system crash could introduce a serious temperature excursion). Also, the computer overhead (i.e., the frequency with which the computer samples data might have to be reduced to accommodate the temperature control function) must be considered.

- Control on/off devices such as the beam stop. An evaluation of the computer's reliability and the effect on system overhead needs to be made prior to implementation.

- Sense the status of devices such as beam stop or pump operation. This type of enhancement can readily be incorporated when the need exists.

- Transfer data to the PDP11/60 and/or VAX installed in an adjacent room at the University of Washington's Nuclear Physics Laboratory. The hardware to accomplish this task is available through serial interfaces and the programming can be performed as the need arises. The primary use of this communication would most likely occur during post-test analys is (e.g., use of disk space, plotter, line printer, or graphics terminal).

Other enhancements are expected to be required once the system is installed and operating experience is acquired. Fortunately, the system is versatile and most needs can be met provided that full consideration is given to the trade-offs involved. 


\section{PROGRAM DESCRIPTION}

The real-time data acquisition program was run in foreground and written entirely in MACRO. All other programs were run in background and, for the most part, written in FORTRAN. A summary of these programs is given in Table 1.

\subsection{DACØ4 (Data ACquisition, Revision 04) DESCRIPTION}

DACD4 performs al1 real-time functions and is run in foreground. The program is basically driven on an interrupt basis from an internal programmable clock operating at a programmed frequency of $100 \mathrm{kHz}$. The clock interrupts the program at an interval entered from the keyboard. It then branches to a clock service interrupt routine which scans the desired $A D C$ channels and stores the data in memory before returning to the section of code from which it was interrupted. In this manner, many other peripheral functions can be performed in either background or foreground without impairing the flow of analog input data at a periodic frequency. Once DACD4 is started and initialized, it executes a portion of the foreground code until it is clock interrupted, at which point it finishes the current instruction and branches to the interrupt service routine. Once the clock interrupt has been serviced, the computer resumes execution of the main foreground program. This process can be repeated many times until eventually the foreground program reaches a TWAIT (timed wait) instruction, which suspends further foreground operation although the clock interrupts continue to occur. When foreground is suspended in this manner, background code is permitted to run for the suspension period (i.e., between clock interrupts). Once the suspension period (set at $1.67 \mathrm{sec}$ ) is over, the foreground program continues at the instruction following the TWAIT. In this manner a system of priorities and timing is established between the clock interrupt routine, foreground code, and background code.

The DACD4 program is divided into five major sections as follows:

(1) Initialization Section

(2) Main Foreground Section

(3) Clock Interrupt Service Routine

(4) Completion Routines

(5) Message and Data Storage Sections 
TABLE 1. Summary of Software Programs

$\frac{\text { Name }}{\text { DACD4 }} \quad \frac{\begin{array}{c}\text { Current } \\ \text { Version }\end{array}}{\text { 19-Ju1-79 }} \quad \frac{\begin{array}{c}\text { Source } \\ \text { Language }\end{array}}{\text { MACRO (a) }}$

Description

A real-time data acquisition program interrupted at a user-selected frequency to scan up to 24 channels of analog-todigital input data. Other services performed include alarms and auto ranging, etc.

GRAFTR 19-JU1-79 FORTRAN ${ }^{(b)}$

A program which is capable of accessing transient data buffers stored by the foreground program and displaying the data graphically.

GRAFAV 19-Jul-79 FORTRAN $(\mathrm{b})$

A program which accesses averaged data on disk and displays it graphically.

TYPAV 19-JUT-79 FORTRAN $(b)$

A program which accesses averaged data on disk and displays it in tabular form at the terminal.

TYPLOG 14-Jun-79 FORTRAN (b) A program designed to write and store text data on disk for retrieval at a later date.

RETXT 14-JUN-79 FORTRAN (b)

A program designed to read text data from disk.

REINT3 19-JU1-79 FORTRAN ${ }^{(b)}$

A program designed specifically to read initialization data associated with DACD4.

RESET 14-Sep-79 FORTRAN (b)

GETGAI 10-May-79 MACRO ${ }^{(b)}$

TIMDAT 19-JUT-79 MACRO ${ }^{(b)}$

DECDAT 15-Jun-79 MACRO $(b)$

ULIB 16-Mar-78 MACRO $(a, b)$

ULIB1 15-Jun-79 MACRO ${ }^{(b)}$
A program which resets graphic display. Subroutine designed to return the gain, given the gain code. Called by GRAFAV and TYPAV.

A FORTRAN callable routine which prints the current time and date.

A program designed to decode and display the date at the terminal.

Set of library routines used extensively in DACO4 and to a lesser extent in background programs.

A library consisting of ULIB plus GETGAI, TIMDAT, and DECDAT. (a) Run in foreground

(b) Run in background 
The initialization section contains the code that queries the user and accepts the initialization data that the code requires to run. This section also writes the initialization data block (DATA.INT) and the text data block (DATA.TXT) to disk. The user enters from the keyboard the following parameters:

- auto restart option

- clock interrupt period in msec (typically 10)

- data file extension (typically A)

- text data (up to 510 characters)

- number of channels/scan (maximum of 30)

- channel/gain code for each channel

- fixed gains

- alarm set points

- auto ranging option

- number of data words to be averaged.

The initialization section is also responsible for printing various error messages, performing calculations and displaying useful information relative to program startup at the console terminal. In the event that the auto restart option is selected, the code reads the required data from a previously created initialization file.

The main foreground section is that MACRO code immediately following the initialization section. Tasks performed within the foreground main section tend to be repetitive, but at a much slower rate than the clock interrupts. For example, the writing of data to disk is perhaps the most important function performed in this section. The functions are performed in the order given below:

1. Test to see if a memory buffer is full and ready to be written to disk. If full, perform the following, otherwise go to Step 2.

a. Compute averaged data.

b. Adjust programmable gains as required if auto gain option is selected.

c. Get date and time of day.

d. Write a block (256 words) of data to disk.

e. Check digital input and messages from terminal. 
f. Print data table at console if request is pending.

g. Alter number of data words averaged if request pending.

h. Check for data outside alarm limits.

i. Go to Step 4 .

2. Check for message from background program (i.e., request for transient data). If message not received, post request and go to Step 4.

3. If data have been received, send transient data to background (GRAFTR).

4. TWAIT (timed wait): Suspend foreground for specified period and return to Step 1.

Thus, the foreground program executes in a repetitious fashion with a period equal to the execution time of the code plus the TWAIT period ( $1.67 \mathrm{sec}$ ). This period must be short compared to the period between disk writes so that a11 data gets stored on disk. The foreground main section also switches disks and increments the file name extension as each disk fills.

The clock interrupt suspends the currently executing code and causes the program to branch to the clock interrupt service routine. This occurs at a frequency specified by the user. Within the service routine, analog-to-digital conversion is initiated and the resulting data are read (approximately 50 usec later) for each of the desired channels and at the desired programmable gain level. The ADC is operated in the random mode, which means that channels can be sampled randomiy, as opposed to operation in sequential mode in which channels are sampled in ascending order only. Although somewhat greater overhead is incurred in the random mode, significantly greater versatility is achieved. Since the interrupt routine is entered at a typical frequency of $100 \mathrm{~Hz}$ (i.e., a period of $10 \mathrm{msec}$ ), it is imperative that the code within the routine be as short as possible to permit foreground and background ample opportunity to execute. It is for this reason that the alarm limits are compared with averaged data following each write to disk as opposed to within the clock interrupt routine itself.

Completion routines are those sections of code entered following certain input/output (I/0) operations. In DAC $\emptyset 4$ a completion routine is entered once the foreground receives data from background. A flag is set to signal the main section that the background progrem awaits transient data. A second completion 
routine is entered following each write to disk. In this case a flag is cleared. If, however, a write to disk is requested before this flag ciears from the previous write operation, the following message is printed: "Backed up Write to Disk Requests." This is a serious error message and usually signifies that the code is initialized improperly (e.g., the period between disk writes is too short).

The last general section of code includes messages and data storage. Organizationally, it is simpler to segregate these sections to the end. Since foreground programs are loaded high in memory, one advantage of having data storage at the end of the code is that its location is not altered if modifications are made to another section. This is helpful if one wishes to verify the value of data in a particular memory location [e.g., by the online debugging technique (ODT)].

\subsection{GRAFTR (GRAph TRansient Data) DESCRIPTION}

The GRAFTR program is written in FORTRAN and run in background. DAC $\varnothing 4$ must be running concurrently. The code is capable of receiving data from DACD4 in order to display transient data graphically that is stored by DAC $\varnothing 4$. Since transient data are not written to disk and since DAC $\$ 4$ writes over each buffer when ful1, one can graph only the most recent transient data. For each of the first 10 channels scanned, 512 points are stored. This, therefore, requires $5 \mathrm{~K}$ memory storage by DACD4. This quantity of data then allows each graph to be filled (i.e., 512 points/graph).

Although the method of obtaining the data is different, GRAFTR is in most other respects similar to GRAFAV, discussed next. The only other significant difference relates to the method of acquiring and decoding the programmable gain. In GRAFAV the gain codes are read from the appropriate disk block in a byte format and then decoded by a MACRO subroutine called GETGAI. In GRAFTR, however, DACD4 returns the data for each graph desired and an octal gain code digit which FORTRAN is capable of converting to the appropriate gain. Due to the close coupling between DACD4 and GRAFTR, modifications to the communication portion of either code should be performed with care. 
The user input to GRAFTR is similar to that of GRAFAV. The user is asked to input the clock interrupt period. This input permits the program to label the horizontal axis in seconds. If this feature is not desired, simply enter a return to override it. Next the user enters scan number, span, offset, and fixed gain. As with GRAFAV, the user is able to label the $X$ and $Y$ axis and insert a title. In addition, a summary table of programmable and fixed gain is displayed. The current data and time of day is displayed. A "*" is displayed when a graph is completed. As with other programs, this implies the statement "Type 1 to Continue." A simple return will terminate the program. A typical example of the graph produced is shown in Figure 1.

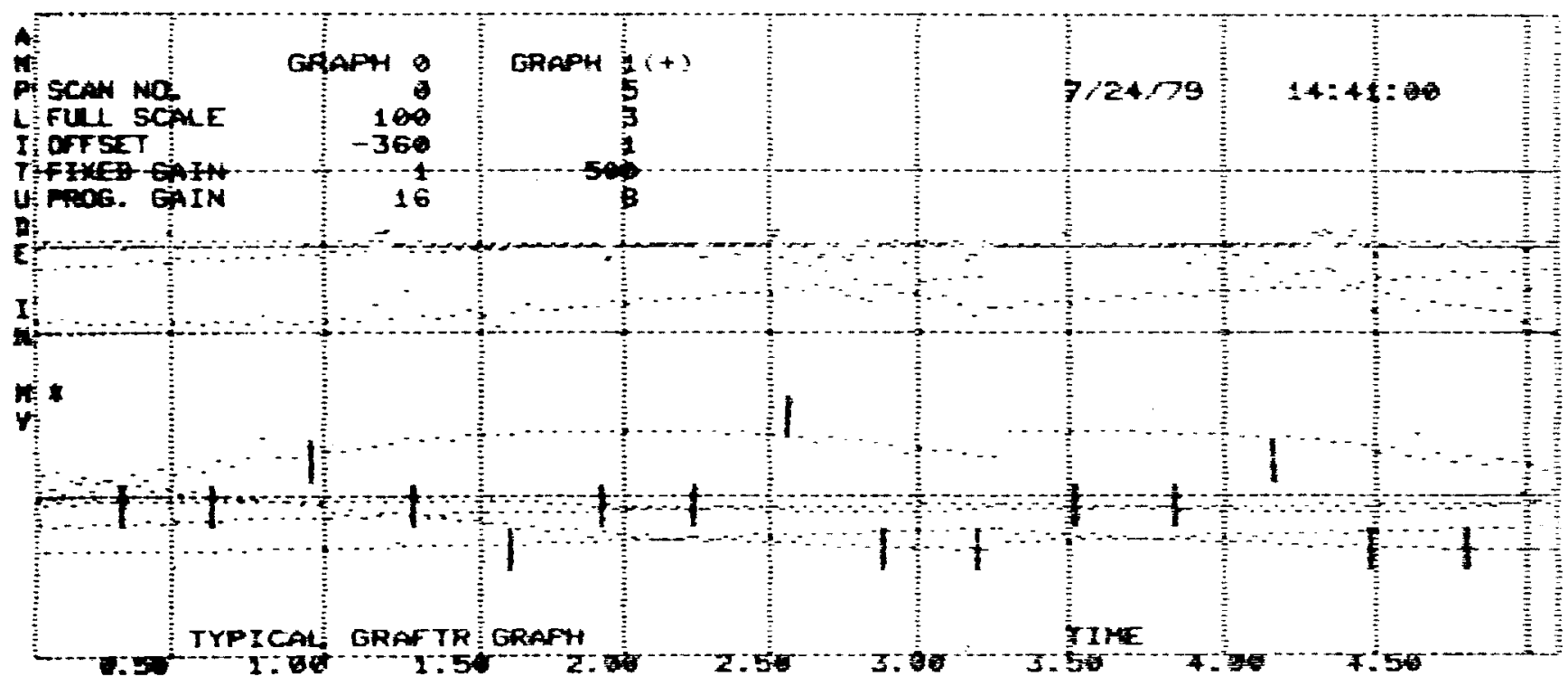

FIGURE 1. Example of Graph Produced by GRAFTR

Several error messages may be displayed. The statements "Error Receiving M Buffer," "Error Receiving N Buffer," and "Error Receiving Gain Codes" mean that an error was encountered during an IRCVD or IRCVDW call. See page 4-53 of the Advanced Programmers Guide for an explanation. (1) The statement "Foreground Job Not Running" occurs when the background program attempts to transmit a data request to the foreground program which is not received. The message, "Gain Code Outside of Range 1-7?", indicates that the foreground sent the background 
an incorrect gain code which may signify a logic problem (e.g., as a result of code modification). Finally, if the user attempts to enter a scan number less than 0 or greater than 10 , the code loops back to the start without an explicit error message.

\subsection{GRAFAV (GRAph AVeraged Data) DESCRIPTION}

GRAFAV is a FORTRAN program run in background. This program is capable of accessing binary data (written by DACD4) from disk and displaying it graphically at the graphics terminal. The program can be run with or without DACØ4 in operation. If DACØ4 is running concurrently, the user has the choice of accessing an active (temporary) file or a closed (permanent) file. This feature allows the user to view current data or data written previously.

The program queries the user about the data desired. The user must specify:

- file name

- starting block number

- number of channels/scan

- scan numbers for graph 0 and 1

- span

- offset

- fixed gain.

As an example, the data desired might be located on file, DXØ:DATA.ØøC. (Note: $D X 1$ is assigned as the default disk drive so DATA. $\varnothing \subset$ is equivalent to DXI: DATA.ØØC.) If the user desires to start at the beginning of the file, he would then enter zero for the starting block number.

The number of channels/scan must coincide with that used when DACØ4 generated the data. The program REINT3 may be run to locate this value in the initialization data. It will probably be useful for the user to copy the initialization data produced by REINT3 for future reference.

Next the user must enter the scan numbers for each graph desired. Scan numbers refer to the order in which channels are sampled. For example, the user can arrange to have channel 2 sampled as the 30th channel scanned. Therefore, the scan number is 30 and the channel number is 2. Scan numbers are 
strictly a function of the software; whereas, channel numbers are hard-wired as part of the hardware.

Finally, the span, offset, and fixed gain must be entered for each of two graphs where the span and offset are in millivolts. Span is always the value in mitlivolts from the bottom to top of the graph; whereas, offset is the value in millivolts at the lower edge of the graph. The program is capable of reading and correcting for programmable gain, but the user must supply the fixed gain. Fixed gain can also be acquired by running REINT3.

Once the prerequisite data are entered, the code reads the disk and draws two graphs. Each point on the graph represents an averaged data value converted to millivolts. Since the graphics hardware has the capability of displaying 512 points along the horizontal axis, the time span depends upon the period over which each data point was averaged. The $X$ axis is divided into vertical grids at 50-point intervals to allow a time base to be identified. For example, if a clock interrupt period of $10 \mathrm{msec}$ is chosen with 1000 points averaged, each averaged point represents $10 \mathrm{sec}$ and the distance between each grid (50 points) represents $500 \mathrm{sec}$ or $8.33 \mathrm{~min}$.

Once the two graphs are drawn, Graph 1 is identified with short vertical markers along its length. The graph is identified with the current date and time, the data starting time and date; and starting block number. The data start time and date corresponds to the time and date recorded in the first block requested. In addition, a summary table is printed showing the fixed and programmable gain for each channel selected.

Finally, the user is queried and given the opportunity to enter a $X$-axis label, Y-axis label, and title. A sample graph is shown in Figure 2. The horizontal grids divide the graph into four equal parts vertically. Thus, if a span of 20,000 were selected with an offset of $-10,000$, there would be 5 volts between horizontal grids with the zero axis at the center (i.e., -10 volts to +10 volts).

Various error codes are reported by the program. For example, the code might print "IREAD ERROR CODE $=-2$ ". The IREAD refers to a programmed request listed alphabetically in Chapter 4 of the Advanced Programmer's Guide. 


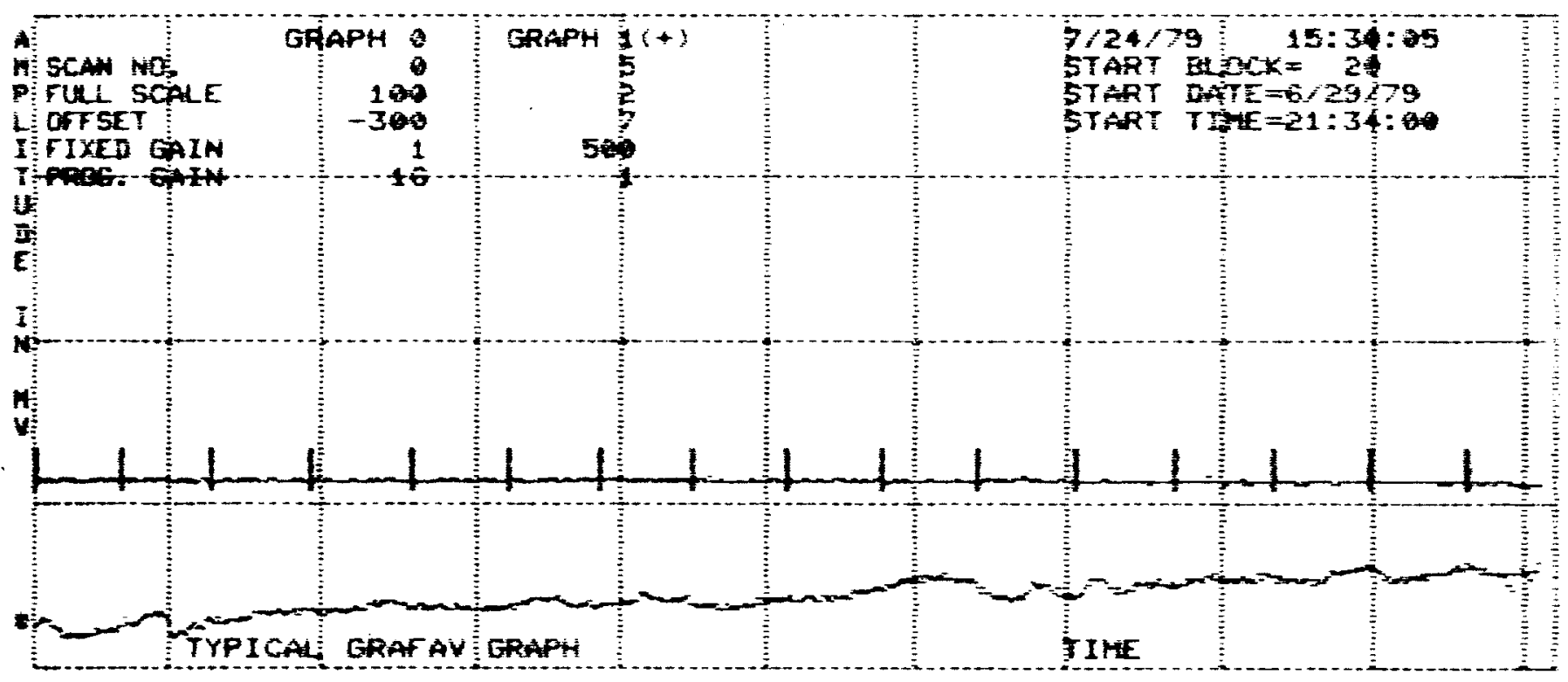

FIGURE 2. Example of Graph Produced by GRAFAV

In this case, the value, -2 , means that a hardware error occurred (see page 4-57). Other error messages involve the ICHCPY (page 4-35), and the LOOKUP (page 4-92) requests.

\subsection{TYPAV (TYP AVeraged Data) DESCRIPTION}

The program TYPAV is written in FORTRAN and designed to run in background. It is designed to facilitate the display of averaged data in a tabular format at the terminal. This program, as with GRAFAV, accesses either active (temporary) or inactive (permanent) files. If a permanent file is desired, the file specification must be entered in the correct format (e.g., DX1:DATA. ФDA). Next the starting block number and number of channels/scan must be entered. Next the message, "Enter 1 to Type First Block Specified", appears. If desired, the code will display all the data in the first block. On the first line the date and time will appear in octal. On the next one to three lines (12 values/line) the octal channel/gain status codes are displayed in the same format entered into DACD4. In fact, they will be equivalent if the auto gain option was not selected. Below the status codes will appear the data values formatted for 12 values/line. These values are simply the digital representations of the numbers stored on disk and, therefore, have not been converted to millivolts or corrected for gain. 
The second half of TYPAV enables any two channels to be displayed in millivolts. This gives a direct comparison in tabular format with the data displayed by GRAFAV. For purposes of displaying these data, the scan numbers and fixed gains must be entered. TYPAV decodes the octal channel/gain status codes for the scans selected by calling the MACRO subroutine GEGAI. The data are formatted in six columns with the data word number and block number appearing in the first and last column, respectively. The value of the first scan selected appears with its programmable gain in columns 2 and 3 , respectively. The value of the second scan selected and its programmable gain, likewise, appear in columns 4 and 5, respectively. Error codes and their explanation are identical to those used in GRAFAV (see Section 4.3).

\subsection{TYPLOG (TYPE LOG) DESCRIPTION}

TYPLOG is a short background program written in FORTRAN. The program allows the user to periodically, at will, record text data on disk for purposes of noting important occurances (e.g., tank sparks, beam drifting, equipment failures, etc). The program allows the user to name the file (maximum of six characters plus extension). Some type of name sequencing (e.g., $\operatorname{LOG} \phi 1, \operatorname{LOG} \phi 2$, etc) is recommended since naming a file with a name previously given to a file on the same disk will delete the previous file. The format of the code is such that six lines must be entered. For example, if four lines of text information were entered, the remaining two lines could be entered by entering an additional two returns. A maximum of 80 characters may be entered per line (limitation of VT55 terminal).

Several error messages can be generated by TYPLOG. Both IENTER and IWRITW error codes can be reported. The user should consult page 4-42 and 4-80 of the Advanced Programmer's Guide, respectively, for an explanation of these error codes. (1) In the case of IENTER, an error code equal to - 1 signifies that the channel is already in use and a specific error message is displayed. This error should not normally occur since the program first identifies an unused channel through the programmed request IGETC. As with several of these programs, the code will loop back if a file name specification is in an incorrect format. This error is detected by an error code not equal to zero for the ICSI call (page 4-36 of Advanced Programmer's Guiro). 


\subsection{RETXT (REad TeXT Data) DESCRIPTION}

RETXT is a short background program written in FORTRAN. The program is designed to read ASCII text data files generated by DACD4 (i.e., DATA.TXT) and TYPLOG. Since both of these text files are one block in length, the code reads only block 0 of the file specified. It could, of course, be easily modified to read text data files of any length.

As with TYPLOG and other programs, the programs loop back if an incorrectly formatted file-name specification is entered. The error message, "Not Enough Que Space", is printed should the code not have enough input/output channels to read the disk. See IQSET (page 4-52 of Advanced Programmer's Guide) for a detailed explanation. (1) LOOKUP and IREAD error codes are reported. LOOKUP error code equal to -2 is specifically reported as, "Cannot Find File Specified on Device" (see Advanced Programmer's Guide for IREAD and LOOKUP error codes). (1) The message "Fatal Read" is reported if an IWAIT error code is generated following an IREAD (see page 4-80 of the Advanced Programmer's Guide for an explanation of possible IWAIT errors).

\subsection{REINT3 (REad INiTialization Data, Revision 3) DESCRIPTION}

REINT3 is a FORTRAN program run in background. The program is specifically designed to read the initialization data file (DATA.INT) generated by the current version of DAC 04 . The data are displayed in a format which would be meaningless for any other type of data. The user need only enter the file name, DATA.INT, after which the disk is read and initialization data are displayed. The first and second line display the date and time of day when DAC 04 was initialized. The next four lines list parameters of potential interest. Each parameter is displayed following the symbol used in DACØ4. The meaning of each parameter is given in Table 2.

Following the table of values, REINT3 types 24 fixed gain values. Each value is written below the channel number (i.e., 0 through 23). Next, REINT3 types 10 pair of alarm values. Each pair is given below the scan number (i.e., 0 through 9). The high limit is given before the low limit. Next REINT3 types the channel/gain status codes (CHNSTA) in octal in the same format 


\section{TABLE 2. Summary of Parameters in REINT3}

\begin{tabular}{|c|c|c|}
\hline Symbol & Display & Description \\
\hline NUMBCH & digital & The NUMBer of CHannels/scan (max of 30$)$; user selectable \\
\hline TRNDAT & digital & The number of TRaNsient DATa channels (set to 10) \\
\hline NUMAVG & digital & $\begin{array}{l}\text { The NUMber of data values over which AVerage is made } \\
(\min =200 ; \max =32,000)\end{array}$ \\
\hline REPAVG & digital & $\begin{array}{l}\text { The number of REPeated AVeraGed data scans per disk } \\
\text { block }\end{array}$ \\
\hline AVGSIZ & digita? & $\begin{array}{l}\text { The AVeraGed data SIZe in words per disk block (NUMBCH } X \\
\text { REPAVG) }\end{array}$ \\
\hline TIM & octal & $\begin{array}{l}\text { High and low order tIMe (double percision) value of } \\
\text { suspension period in tics (TWAIT) }\end{array}$ \\
\hline AUTOGN & digital & $\begin{array}{l}\text { AUTO GaiN option. If value }=1 \text { auto gain is selected, } \\
\text { otherwise fixed programmable gain }\end{array}$ \\
\hline CLOCK & digital & CLOCK interrupt period in msec \\
\hline DRCSR & octal & DRV-11 (ADC interface) Control Status Register address \\
\hline OUT & octal & DRV-11 OUTput register (for sending data to ADC) address \\
\hline INP & octal & $\begin{array}{l}\text { DRV-11 INPut register (for receiving data from } A D C \text { ) } \\
\text { address }\end{array}$ \\
\hline CSR & octal & Programmable Clock Control Status Register address \\
\hline BPR & octal & Programmable Clock Buffer/Preset Register address \\
\hline DGOUTI & octal & DiGital OUTput No. I address \\
\hline DGOUT2 & octal & DiGital OUTput No. $\underline{2}$ address \\
\hline DIGINP & octal & DIGital INPut address \\
\hline DATE & octal & DATE code when DAC $\varnothing 4$ was initialized \\
\hline TOD & octal & $\begin{array}{l}\text { Time of Day in tics past midnight when DACD4 was } \\
\text { initialized (high-order part) }\end{array}$ \\
\hline TODI & octal & $\begin{array}{l}\text { Time of Day in tics past midnight when DACD4 was } \\
\text { initialized (low-order part) }\end{array}$ \\
\hline
\end{tabular}


as entered in DACQ4. The program fills in unused codes with 0 up to the maximum of 30 . Since 0 is a iegitimate gain code (channel 0 with a gain of 1 ), the user should use the value of NUMBCH to determine how many codes are meaningful.

Possible error messages are similar to previous programs described. Error codes are reported for LOOKUP and IREAD programmed requests (see pages 4-92 and 4-56, respectively, of Advanced Programer's Guide). (1) The LOOKUP error code of -2 is specifically reported as "Cannot Find File Specified on Device." A "Fatal Read" message is displayed if the IWAIT generates an error code following an IREAD cal1. (See page 4-80 of Advanced Programmer's Guide). (1) Also, a "Not Enough Que Space" message is displayed if insufficient input/output channels are available to read the disk (page 4-52).

\subsection{RESET (RESET Graphics Display) DESCRIPTION}

RESET is a short FORTRAN program run in background. RESET is designed to clear the graphics display. No error messages are reported by RESET.

\subsection{GETGAI (GET GAIn) DESCRIPTION}

GETGAI is a short MACRO subroutine callable from FORTRAN. The FORTRAN program supplies GETGAI with a channel/gain status code and GETGAI returns the gain. This operation is efficiently performed in MACRO since bit manipulation of the channel/gain status code is required. The following format as used in GRAFAV is shown below:

Ca11 GETGAI [CHNSTA(ICHø+1), IPRGNø]

where: CHNSTA is the array containing the channel/gain code ICH $\emptyset$ is the scan number for graph 0 IPRGN $\emptyset$ is the gain value returned for graph 0 . No error messages are generated by GETGAI. 


\subsection{TIMDAT (Get TIMe and DATe) DESCRIPTION}

TIMDAT is a MACRO subroutine callable by FORTRAN which in turn calls the TIMOUT MACRO subroutine in ULIB (Utility LIBrary). TIMOUT displays the current time of day and date at the terminal. This chain is necessary since subroutines in ULIB are only callable from MACRO (with the exception of FILINP). No error messages are generated by TIMDAT.

\subsection{DECDAT (DECode DATe) DESCRIPTION}

The program DECDAT is a short MACRO routine callable from FORTRAN. The code will output an ASCII date string to the terminal given a date code (see page 2-47 of Advance Programmer's Guide for information on date code). (1) The FORTRAN calling program uses the following format:

CALL DECDAT (IDATE)

where IDATE contains the date code. The ASCII string output to the terminal is in the following format: 6/15/79. This function is most efficiently performed in MACRO since bit manipulation of the date code is required. DECDAT calls the subroutine DIGOUT described in the next section. GRAFAV, TYPAV, and REINT3 make use of DECDAT by displaying the date stored in the first disk block specified. This subroutine is to be distinguished from the program TIMDAT, which outputs the current date and time stored by the RT-11 monitor. No error messages are generated by DECDAT.

\subsection{ULIB (Utility LIBrary) DESCRIPTION}

ULIB is a collection of MACRO callable subroutines compiled in a library which can be linked to a user's program. DAC $\emptyset 4$ makes extensive use of several of these programs. As described in previous sections, the MACRO program, TIMDAT, and DECDAT use ULIB.

In general, the subroutines contained within ULIB are called by the MACRO statement:

JSR R5, SUBROUTINE

Where SUBROUTINE is the name of the program. Arguments are passed on general 
register $R \emptyset$. The terminal bell is activated to signal that the program awaits data. Overflows or illegal characters in any of the input generate error messages. All programs, except DFIX and DFLOAT, use single precision integers and/or real numbers. A brief description of each subroutine is listed in Table 3.

\section{TABLE 3. Subroutines Contained in ULIB}

$\frac{\text { Name }}{\operatorname{OINP}^{(a)}}$
$\operatorname{OOUT}^{(a)}$
$\operatorname{DIGIN}^{(a)}$
$\operatorname{DIGOUT}^{(a, b)}$
$\operatorname{FLOAT}^{(a)}$

FIX

SQRT

\section{Description}

Octal INPut. An octal number (0-177777) is brought in from the terminal and stored in $R \emptyset$ in binary.

Octal OUTput. A binary number, stored in $R \emptyset$ is output to the terminal in octal. Leading zeroes are included.

DIGital INput. A digital integer (single precision) is brought in from the terminal and stored in $R \emptyset$ in binary form.

DIGital OUTput. A binary number in $\overline{R \emptyset}$ is output to the terminal in digital form. Leading zeroes are ignored.

FLOATing point. An integer in $R \emptyset$ is converted to floating point format and returned in $R \emptyset$. The integer in $R \emptyset$ is lost.

A real number, the address of which is stored in $R \emptyset$ is converted to integer form after rounding off. Positive or negative overflows are flagged and 77777 (octal) is returned. The resulting integer is stored in $R \emptyset$.

SQuare RooT. The square root of a real number, the address of which is stored in $R \emptyset$, is calculated and the address of the result is returned in $R \emptyset$. If the number pointed to by $R \emptyset$ is negative, a NSQRT message is typed and the program is terminated. 
TABLE 3. Subroutines Contained in ULIB (contd)

$\frac{\text { Name }}{\text { TIMOUT }^{(a, c)}}$

$\mathrm{LN}$

FLTIN

FLTOUT ${ }^{(a)}$

DFIX

DFLOAT $^{(a)}$

\section{Description}

TIMe OUTput. The current time of day and date are output at the terminal. This program uses the line clock.

Natural Log. The natural $\log$ of a real number, the address of which is located in $R \emptyset$, is calculated and the address of the answer is returned in $R \emptyset$.

FLoaTing point INput. A real number is input from the terminal and the address of the result is stored in $R \emptyset$. The format of the input is:

$F=N N \cdot$ NNNNE $\pm N N$

where $|F|<1.7 \times 10^{38}$. The number is assumed positive unless a minus sign precedes all other characters. A leading + is not allowed. The decimal point may be located anywhere in the number or it may be omitted, in which case it will be assumed after the last number before the exponent. $E+N N$ implies the exponent 10. $E+N$ and $E N$ are also allowed, where the space implies a + sign.

FLoaTing point OUTput. A real number, the address of which is in RD, is output to the terminal in E12.5 format.

Double precision FIXed point. A single precision floating point number, the address of which is in $R \emptyset$ is converted (after rounding off) to a double precision integer. The address of the result is passed to the calling program in $R \emptyset$.

Double precision FLOATing point. A double precision integer (address in $R \emptyset$ ) is converted to a single precision floating point number (address

returned in $R \emptyset$ ). 
TABLE 3. Subroutines Contained in ULIB (contd)

Name

FILINP

\section{Description}

FILe INPut. Input a filename through terminal. FORTRAN callable. Use JSR PC,FILINP from MACRO.
(a) Used by DACD4
(b) Used by DECDAT
(c) Used by TIMDAT

\subsection{ULIBI (Utility LIBrary Revision 1) DESCRIPTION}

ULIBI is a library consisting of the following object programs:

ULIB (1ibrary of MACRO callable subroutines)

GETGAI (FORTRAN callable subroutine)

TIMDAT (FORTRAN callable subroutine)

DECDAT (FORTRAN callable subroutine)

Its creation was necessitated by a limit imposed by RT-11 on the number of object files which could be linked at once. For example, to link GRAFAV, all of the following object codes would be required:

\section{GRAFAV \\ FORLIB \\ PLOT55 \\ ULIB \\ GETGAI \\ TIMDAT \\ DECDAT}

where ULIBI should be substituted for the last four codes listed. As new subroutines are developed, new libraries may be created (e.g., ULIB2, etc). 


\section{PROGRAM OPERATION}

The operation of the programs previously discussed is intended to be selfexplanatory in most instances provided that the user is generally familiar with the RT-11 operating system. The input to DACD4 is, of course, critical to its proper operation and, therefore, one or more thermal creep tests should be conducted to evaluate the choice of input. These tests may also form the basis for some enhancements and/or modifications prior to the first irradiation creep test. The thermal creep tests will also yield valuable data relative to the reliability of the computer and its peripherals. As discussed earlier, the value of several anticipated enhancements depends largely upon the computer's reliability (e.g., freedom from system crashes).

Prior to operating DACD4 the foreground/background monitor must be booted in. Several monitor commands have been included as part of STARTF.COM, which are executed automatically after the system is booted. They could likewise be executed from the keyboard. The commands are:

ASSIGN DXI:DK

SET TT:SCOPE

SET USR NOSWAP makes disk drive 1 the default disk modifies operation of delete key makes USR resident in memory

These commands are described fully within the RT-1l documentation. (See Chapter 4 of System User's Guide.) $)^{(2)}$ In addition to booting in the system, the user must identify which programs should be available and on which disk drive. It is anticipated that early operating experience will largely dictate the requirements, depending primarily upon the types of operations that will be performed in background during creep testing. (See Chapter 2 of System Generation Manual for general recommendation.) ${ }^{(3)}$ An outline of the programs that may be resident on disk is given in Tables 4 and 5 .

The programs listed in the first two categories of Table 4 can easily be fit onto a single disk to facilitate booting the system and execution of DACD4. Disk drive 0 is recommended for this purpose since data are initially written to drive 1 . 
TABLE 4. Generaliy Useful Programs

RT-11 Operating System:

\begin{tabular}{lccl}
\multicolumn{1}{c}{ Name } & Blocks & & \multicolumn{1}{c}{ Description } \\
DXMNFB.SYS & 74 & & Foreground/background monitor \\
SWAP.SYS & 24 & & External monitor swap blocks \\
STARTF.COM & $\frac{1}{99}$ & Command file
\end{tabular}

To Run DACФ4:

DACØ4.REL 41 Real-time data acquisition program

Very Useful:

$\begin{array}{lll}\text { DIR.SAV } & 17 & \text { Lists files on disk } \\ \text { DUMP.SAV } & \frac{7}{24} & \text { Shows binary data on disk }\end{array}$

Useful:

$\begin{array}{lll}\text { PIP.SAV } & 16 & \text { Transfer and copy files } \\ \text { DUP.SAV } & \frac{21}{37} & \text { Misc. disk file operations }\end{array}$

Background Data Trend Analysis:

$\begin{array}{lrl}\text { GRAFAV.SAV } & 32 & \text { Graph averaged data } \\ \text { GRAFTR.SAV } & 31 & \text { Graph transient data } \\ \text { TYPAV.SAV } & 34 & \text { Tabulate averaged data } \\ \text { REINT3.SAV } & 22 & \text { Read initialization data } \\ \text { RETXT.SAV } & 18 & \text { Read text data } \\ \text { TYPLOG.SAV } & 18 & \text { Write text data to disk } \\ \text { RESET.SAV } & \underline{10} & \text { Clear graphic display }\end{array}$


TABLE 5. MACRO and FORTRAN Development Programs

Name Blocks

MACRO Program Development

$\begin{array}{lrl}\text { EDIT.SAV } & 19 & \text { Modify source code } \\ \text { MACRO.SAV } & 45 & \text { Assemble MACRO code } \\ \text { LINK.SAV } & 29 & \text { Link object codes } \\ \text { SYSMAC.SML } & 37 & \text { Default MACRO library } \\ \text { ULIB.OBJ } & \underline{12} & \text { User 1ibrary }\end{array}$

FORTRAN Program Development:

$\begin{array}{lrl}\text { EDIT.SAV } & 19 & \text { Modify source code } \\ \text { FORTRA.SAV } & 181 & \text { Compile FORTRAN code } \\ \text { LINK.SAV } & 29 & \text { Link object codes } \\ \text { SYSLIB.OBJ } & 42 & \text { Default FORTRAN library } \\ \text { FORLIB.OBJ } & 151 & \text { FORTRAN library } \\ \text { PLOT55.OBJ } & 3 & \text { Graphic subroutines } \\ \text { ULIBT.OBJ } & \underline{13} & \text { User library } \\ & 438 & \end{array}$

As can be seen there are many programs with large space requirements. Since each disk has an effective storage space of 484 blocks (assuming two blocks for the directory) and since space must be reserved for data storage, compromises must be made. It is suggested initially that a foreground/background system disk be inserted into drive 0 and used to boot the system up and run DACØ4. A disk with the background trend analysis programs would be inserted into drive 1 with data storage on the remaining blocks. It would also be desirable to include DIR and DUMP and possibly PIP and DUP if the need evolves. The inclusion of either the MACRO or FORTRAN development files for use during tests is not recommended initially since: 
1. The space consumed is large and reduces the data storage capacity of the disk.

2. Program development in background increases the probability of a system crash or foreground program termination. The probability of these occurrences, however, may be small for the experienced user who is careful to avoid incorrect input (e.g., fatal monitor errors).

It would be desirable in a long test that requires more than two disks for the user to prepare two sets of disks in advance. The first set would be properly initialized disks for drive 0 . The second set would have the necessary background files and be squeezed to achieve a contiguous free space for data storage (see Chapter 4 of System User's Guide for a description of a11 interactive commands including the SQUEEZE command) for use in drive 1.

As part of the initialization process it might be useful to develop a identification system. In this manner up to 12 characters can be stored in the directory as a permanent record, in addition to the user's name. (See pages 4-77 of System User's Guide for VOLUMEID Option.) ${ }^{(2)}$ As an example:

.DIR[CR] ${ }^{(a, b)}$

DIR-F-ITlegal directory

INIT/SEG:2/VOLUMEID[CR] Device? DXI:

$\mathrm{DX} 1: /$ Init are you sure?Y[CR]

Volid?TEST 24-3-35[CR]

Owner Name?J.DOE[CR]

.DIR/VOL[CR]

Volume ID: Test $24-3-35$

Owner: J. Doe

O Files, 0 Blocks

484 Free Blocks
Check to be sure it is an unused disk

Initialize with two blocks reserved for directory and Volume ID requested

Label volume ID

Input user's name

Check for errors

(a) [CR] indicates a carriage return entered by striking the return key

(b) Underlined characters are entered by user; all others are generated by computer. 


\subsection{DACØ4 OPERATION}

Once the foreground/background monitor is booted up, DAC $\emptyset 4$ can be run.

The first step is to set the current date and time as follows:

.DATE 12-May-79[CR]

Enter date

.DATE[CR]

Check date

12-May-79

.TIME 8:15[CR]

Enter time

.TIME[CR]

Check time

08:15:09

Next, assuming DACØ4.REL is resident on drive 0 and a data disk is loaded into drive 1, type:

.FRUN DX:DAC $\emptyset 4[C R]$

$\mathrm{F}>$

HAS DATE AND TIME BEEN PREVIOUSLY ENTERED? IF NOT RESTART.

ENTER CTRL/F FIRST

TYPE I FOR AUTO RESTART

CTRL/F[CR] ${ }^{(a)}$

ENTER CLOCK INTERUPT PERIOD (10 MS TYPICAL; 320 MS MAX)

The response $F>$ on the first line indicates that a message from the foreground follows. The second line reminds the user to enter the date and time if not done previously. The third line reminds the user that a CTRL/F (simultaneously push CTRL and $F$ keys) must be struck before proceeding to inform the computer that any characters that follow are intended for the foreground job. The fourth line gives the user the option of an automatic restart. If selected, the code reads the initialization file, DATA.INT, in lieu of accepting user input. As shown a carriage return was struck in lieu of the 1 needed for the auto restart option. The last line asks the user to enter the clock interupt period. There are a number of considerations which will undoubtedly influence the choice of this parameter. Ten milliseconds is recommended initially. Therefore, the user entry continues as follows:

$\underline{10[C R]}$

(a) CTRL/F is entered by simultaneously striking the CTRL and F key 


\section{ENTER DATA FILE EXTENSION (TYPICALLY = "A")}

As indicated, the user is asked to enter the data file extension. Since the code is set up to increment the extension automatically, the letter " $A$ " is recommended. However, if the code is restarted (either by manual input or automatic restart), the user should enter the next unused extension so that the data file extensions will be continuous. Therefore:

A[CR]

293 = NUMBER OF FREE BLOCKS ON DISK

ENTER TEXT: LIMIT = 510 CHARACTERS; TERMINATE WITH @ SYMBOL

THIS IS A TEST OF DACD4 [CR]

TO DEMONSTRATE ITS [CR]

USE $\mathrm{Q}[\mathrm{CR}]$

ENTER NUMBER OF CHANNELS PER SCAN; MAX $=30$

The user is given the free disk space to judge if the disk has been properly initialized. The "Q" symbol informs the program that the text string is terminated. The user should not type more than 80 characters per line and limit the message to 510 characters. After the message is received, the program requires the number of channels/scan. This will, of course, usually correspond to the number of hardware channels wired up unless the user wishes to sample one or more channels more than once. This may be appropriate under one of the following conditions:

1. The auto gain option is not desired and so a single hardware channel is scanned several times with different programmable gain settings. This use might be appropriate for transient analysis since programmable gain is only checked at the frequency of writing to disk.

2. A time correlation is critical. For example, the temperature is required at the instant the strain is to be measured in order to correct for thermal expansion. Therefore, the same thermocouple might be read just prior to and just after the strain reading. 
3. Statistical analysis may show that more readings would significantly improve accuracy. Therefore, critical readings might be read several times during each scan.

In addition to the above considerations, the user might consider the efficiency with which disk space is used. Each disk block is only filled in increments of complete scans; therefore, blank space can exist, the amount of which depends upon the number of channels/scan. The blank space is calculated by recognizing that there are 256 words per disk block. The first three words are used by the date ( 1 word) and time ( 2 words). The channel/gain status codes then use $X / 2$ words, where $X$ equals the number of channels/scan. If $X$ is odd, the remainder is rounded up to the next whole number of words. Thus, both 29 and 30 channels/scan use 15 words to store the channel/gain status codes. The remaining space is then available for an integral number of complete scans. In the case of 29 channels/scan we have:

$256-3-15=238$

words available in which eight complete scans of 29 words each will fit, taking a total of 232 words and leaving 6 unused blanks. This turns out to be considerably more efficient than 30 scans/channels, which only permits seven scans per disk block and results in 28 blank spaces. Some cases have been summarized in Table 6 . The entry continues as follows:

$\underline{21[C R]}$

ENTER CHANNEL/GAIN CODE FOR NUMBER OF CHANNELS SELECTED

STATUS $=X X Y$; WHERE $X X=O C T A L$ CHANNEL NO. $Y=O C T A L$ GAIN CODE

The user must, therefore, enter in this example 24 codes, each of which designates a hardware channel and gain code. Gain codes are interpreted as shown in Table 7. 
TABLE 6. Summary of Disk Space Utilization

\begin{tabular}{|c|c|c|c|c|}
\hline Channels/Scan & $\begin{array}{l}\text { Data Space } \\
\text { Available } \\
\end{array}$ & Scans/Block & $\begin{array}{c}\text { Data Words/ } \\
\text { Block }\end{array}$ & $\begin{array}{c}\text { Unused } \\
\text { Space, Words }\end{array}$ \\
\hline 10 & 248 & 24 & 240 & 8 \\
\hline 20 & 243 & 12 & 240 & 3 \\
\hline 21 & 242 & 11 & 231 & 11 \\
\hline 22 & 242 & 11 & 242 & 0 \\
\hline 23 & 241 & 10 & 230 & 11 \\
\hline 24 & 241 & 10 & 240 & 1 \\
\hline 25 & 240 & 9 & 225 & 15 \\
\hline 26 & 240 & 9 & 234 & 6 \\
\hline 27 & 239 & 8 & 216 & 23 \\
\hline 28 & 239 & 8 & 224 & 15 \\
\hline 29 & 238 & 8 & 232 & 6 \\
\hline 30 & 238 & 7 & 210 & 28 \\
\hline
\end{tabular}

TABLE 7. Gain Code Interpretation

Gain Code Gain

$\begin{array}{rr}0 & 1 \\ 1 & 1 \\ 2 & 1 \\ 3 & 2 \\ 4 & 4 \\ 5 & 8 \\ 6 & 16 \\ 7 & 32\end{array}$


The octal channel number is simply the channel number expressed in octal where the first channel is number 0 and the last channel is number $27_{8}\left(23_{10}\right)$. As a typical entry:

$\underline{0[C R]}$

$60[\mathrm{CR}]$

$140[C R]$

$150[\mathrm{CR}]$

220[CR]

$230[\mathrm{CR}]$

$240[C R]$

$160[\mathrm{CR}]$

$170[\mathrm{CR}]$

$270[C R]$

$10[\mathrm{CR}]$

20[CR]

$30[\mathrm{CR}]$

$40[C R]$

$50[\mathrm{CR}]$

$\underline{100[C R]}$

200[CR]

210[CR]

250[CR]

260[CR]

O[CR]

ENTER 24 ADC FIXED GAIN VALUES

The order of hardware channels is immaterial and gain codes are selected at will. In this example, channel 0 was sampled twice at a gain of one each time. In this example, the programmable gain of each channel was set to 1 since auto gain is to be selected.

Next, the fixed gains are entered for each of the 24 hardware channels as follows: 
I[CR]

[ [CR]

[ [CR]

[CR]

2[CR]

2[CR]

2[CR]

2[CR]

10 [CR]

10[CR]

10[CR]

10[CR]

200[CR]

200[CR]

200[CR]

200[CR]

500[CR]

500[CR]

500[CR]

500[CR]

500[CR]

500[CR]

500[CR]

500 [CR]

ENTER 20 ALARM VALUES (HI/LO) FOR FIRST 10 CHANNELS SCANNED

Fixed gain values may be verified by sliding out each amplifer board approximately $4 \mathrm{in}$. and reading the gain value on each plug-in gain module (a special key hangs on the inside of ADC door for withdrawing boards). The values given above correspond to the installed configuration at delivery.

The amplifer boards are of two types: high level (Model 620060) and low level (Model 620050). High-level boards use fixed gains in the range of 1-10 and low-level boards use fixed gains in the range of 10-1000 (see page 9-10 under General Description in the NEFF Manual for further details). 
Also, the installed filter cutoff is $100 \mathrm{~Hz}$ on all 24 channels as delivered. This value can be changed with a suitable plug-in resister module as was the case with the fixed gain values. Next, the alarm values are entered in millivolts, where the first value is the upper limit and the second value for each channel is the lower limit as follows:

9500[CR]

$-9500[C R]$

$-1000[C R]$

$-3000[C R]$

$25[\mathrm{CR}]$

$15[C R]$

$\underline{30[\mathrm{CR}]}$

10[CR]

10[CR]

5 [CR]

10[CR]

5 [CR]

$10[\mathrm{CR}]$

5[15]

10[CR]

$\mathrm{O}$ [CR]

10[CR]

2[CR]

O[CR]

$-2[\mathrm{CR}]$

TYPE I FOR AUTO GAIN

The 9500/-9500 entry could serve to alarm if a channel went full scale. An $11000 /-11000$ entry would effectively remove an alarm from service since the maximum output the ADC can generate is 10000/-10000. Also, negative signals can be treated as evidenced by the entry, $-1000 /-3000$. Only integers in the range of \pm 32000 are legal.

Experience will largely govern the choice of the auto gain option. It will depend largely on whether the user or computer (as programmed) is judged to 
make the better choice of programmable gain setiing. As programmed, the computer changes gain settings so as to maintain the amplified analog value in the range of 3.5 to 7.0 volts or -3.5 to -7.0 volts. These values were selected as a compromise between good resolution and freedom from saturation during transients. The auto gain option is selected as follows:

I[CR]

ENTER NUMBER OF DATA POINTS TO BE AVERAGED: 200-32000

Next, the number of averages made is entered as follows:

$600[C R]$

6.00000E $\emptyset 1$ = SEC PER DISK WRITE

291 = NUMBER OF FREE BLOCKS ON DISK

$7 / 19 / 79 \quad 14: 35: 23$

Here, 600 was calculated to give 1 min between disk writes or $6 \mathrm{sec}$ per averaged word. For comparison, an entry of 6000 would have given 1 min averaged data values which would result in 10 min per disk write and allow a test period of $80 \mathrm{hr}$ on a blank disk with 484 blocks. Next, the free space is indicated, which is two blocks less than the previous value since DAC 44 wrote a DATA. INT and DATA.TX file, each having one block. This, therefore, completes the entry. The user should observe that the $A D C$ data and channel lights are flashing as an indication of data conversion. Bit 1 will alarm on Digital Output Panel 1 to indicate that drive 1 is in operation (bit 0 indicates drive 0 ). Just after the first write to disk, the data will be checked against the alarm limits input. Alarms are displayed on bits 0 to 9 on Digital Output Panel 2 where bit 0 indicates $\operatorname{scan} 0$, etc.

Certain codes are available to communicate with DAC $\varnothing 4$ from the keyboard and the digital input panel. Of course CTRL/F must first be struck if previous communications were with the background. The codes shown in Table 8 are available.

An example of the data table is shown in Table 9. The number ( $9002 \emptyset 1)$ below the table is the octal representation of the 16-bit digital input word. Bit 7 is set since the table is desired. The value of the word in bits $0-5$ is 1 (one); therefore, the number of words averaged has not been altered from the initialized value. 
TABLE 8. DACø4 Input Codes

Code

Bit 7 Set $^{(a)}$

Bit 7 Cleared $(a)$

อญอ

$\mathrm{CTRL} / \mathrm{C}^{(\mathrm{b})}$

CTRL/C

BREAK

Bit $6 \operatorname{Set}^{(a)}$

Bit 6 Cleared $(a)$

Bits $0-5^{(a)}$
Function

Requests a table of scan number, channel number, fixed gain $(F X)$, programmable gain (PR). Data is displayed in millivolts and automatically updated for each channel scanned. The heading of the table displays the current time and date along with the disk status. The last disk block written is given (note that the number of blocks written is one greater since the first block is zero) along with the number of remaining blocks. Therefore, the sum of both numbers plus one gives the total space available for data storage.

Cancels table requests.

Terminates program after closing current file (i.e., making file permanent and updating directory accordingly).

Terminates program without closing current file (i.e., file name does not appear on directory). This code is part of RT-11 monitor.

Electricaliy disabled within terminal. Otherwise would halt computer (Enable/Halt switch on computer provides the same function). The risk of accidentally striking this key seemed to outweigh its convenience.

Causes number of words averaged to be divided by word in bits $0-5$.

Causes number of words averaged to be multiplied by word in bits $0-5$.

Value of word set determines factor by which number of words averaged is either increased or decreased. Switch up indicates one, zero, otherwise.

(a) Refers to digita? input. Bits set with switch up, cleared otherwise.

(b) CTRL/C is entered by simultaneously striking the CTRL and C keys. 
TABLE 9. Data Table Produced by DACD4

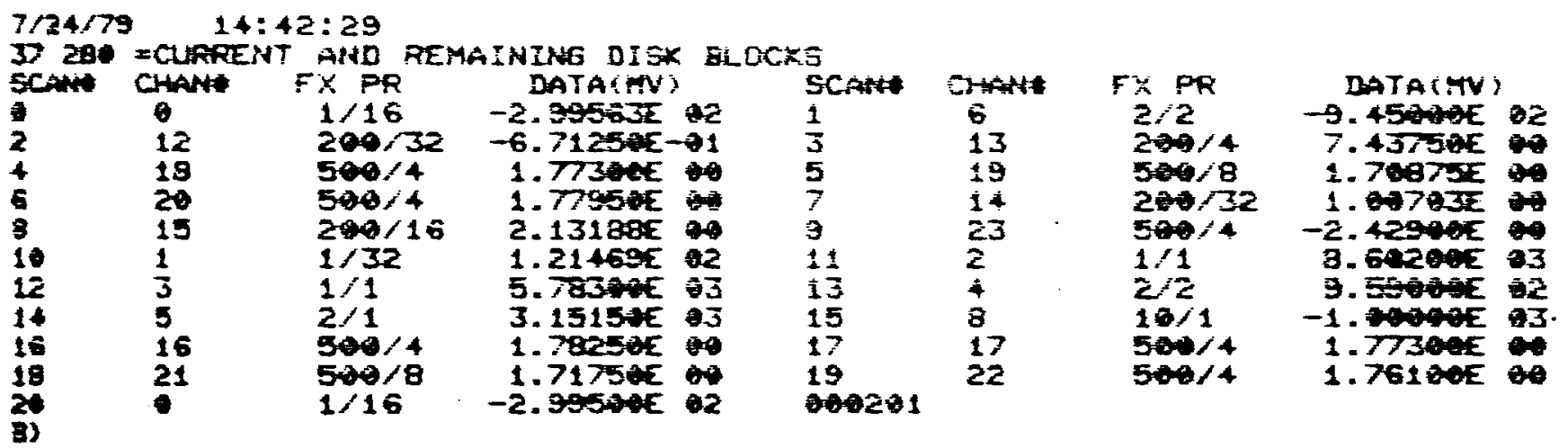

\subsection{GRAFTR OPERATION}

GRAFTR operation is similar in most respects to that of GRAFAV. Of course, DACD4 must be running concurrentiy since it supplies the data for graphic display. Assuming GRAFTR.SAV is resident on drive 1 and DACD4 is operating, simply type:

CTRL/B

.RU GRAFTR[CR]

ENTER CLOCK INTERRUPT PERIOD (MS)

The clock interrupt period is only required to enable the program to label the $X$-axis in seconds. Enter a [CR] if this option is not desired. The entry continues as follows:

$\underline{10[C R]}$

ENTER CHANNEL NO, SPAN (MV), OFFSET (MV), AND FIXED GAIN FOR GRAPH $\emptyset$

O[CR]

$10000[C R]$

$\underline{O[C R]}$

ICR]

ENTER CHANNEL NO, SPAN (MV), OFFSET (MV), AND FIXED GAIN FOR GRAPH 1 $3[\mathrm{CR}]$ 
$12000[C R]$

$-12000[C R]$

I[CR]

TYPE: I TO DELETE GRIDS

where the desired scan numbers were entered. All spans and offsets are in millivolts.

The last message above refers to the optional grids superimposed upon the graph. Preference will dictate the selection. The entry continues as:

[CR]

TYPE: "SPACE, TITLE OF GRAPH"

where the user enters a space by striking the space bar before entering an optional title. A [CR] will delete the title option. Entry continues as: TEST GRAPH OF TRANSIENT DATA[CR]

TYPE: SPACE, LABEL FOR X-AXIS"

TIME[CR]

TYPE: LABEL FOR Y-AXIS"

AMPLITUDE IN MV[CR j

*

where a space is not requred before entering the $Y$-axis label due to the rethod of programing. The * implies, "Type 1 to continue." A [CR] wili terminate GRAFTR. Vertical bars resembling error bars denote graph 1. A "+" symbol following the graph 1 label serves as a reminder. Current date and time are dispiayed. Should a 1 be struck the code continues as follows:

$I[C R]$

TYPE 1 TO OVERRIDE GRAPHIC RESET

The user can type a 1 to preserve the graphic display while entering input for the next graph. From this point on the input is the same as given previousiy. A typical example of the graph produced appeared previousiy in Figure 1. 


\subsection{GRAFAV OPERATION}

The operation of all background programs is largely self-explanatory. Presuming, GRAFAV.SAV is resident on drive 1, simply type:

CTRL/B

.RU GRAFAV[CR]

ENTER 1 TO GRAPH ACTIVE DATA FILE

The CTRL/B is necessary if DACD4 is running concurrently in foreground and if foreground had been receiving messages from the keyboard. The user need not specify the device since DXI is assumed to be the default device. The program responds by asking if an active data file is desired (i.e., the data being generated concurrently by DAC $\$ 4)$. Input proceeds as follows for a permanent file:

[CR]

ENTER PERMANENT FILE NAME

*DATA. $99 A$

ENTER STARTING BLOCK NUMBER

O[CR]

ENTER NUMBER OF CHANNELS/SCAN

$24[\mathrm{CR}]$

1624151

ENTER SCAN NUMBERS FOR GRAPHS $\emptyset$ AND 1

O[CR]

$3[\mathrm{CR}]$

ENTER SPAN, OFFSET, AND FIXED GAIN FOR GRAPH $\emptyset$

20000[CR]

$-10000[C R]$

I[CR]

ENTER SPAN, OFFSET, AND FIXED GAIN FOR GRAPH ? 
$20000[C R]$

$-10000[C R]$

$\underline{\mathrm{I}} \mathrm{CR}]$

TYPE 1 TO DELETE GRIDS

$\underline{1[C R]}$

TYPE: "SPACE, TITLE OF GRAPH"

TEST GRAPH OF DATA. $9 \emptyset A[C R]$

TYPE: SPACE, LABEL FOR X-AXIS"

TIME[CR]

TYPE: "LABEL FOR Y-AXIS"

AMPLITUDE IN MV[CR]

*

The number of channels/scan corresponds to the initialization value used for DAC 04 . The scan numbers refer to the order in which the channels were scanned as opposed to the hardware channel number. Both scan and offset are in millivolts. Once the graph is displayed the "*" implies: "Type 1 to Continue." For example:

[ [CR]

ENTER 1 TO GRAPH ACTIVE DATA FILE

A typical example of the graph produced appeared previously in Figure 2.

\subsection{TYPAV OPERATION}

Assuming TYPAV.SAV is resident on drive 1 it may be run by simply typing:

$\underline{C T R L / B}$

.RU TYPAV[CR]

TYPE 1 TO ACCESS ACTIVE FILE

[CR]

ENTER PERMANENT FILE NAME; DEFAULT EXT $=\emptyset \emptyset B$ 
*DATA [CR]

ENTER STARTING BLOCK NUMBER

20[CR]

ENTER NUMBER OF CHANNELS/SCAN

$21[\mathrm{CR}]$

ENTER 1 TO TYPE FIRST BLOCK SPECIFIED

I[CR]

where the data output appears as shown in Table 10.

ENTER 1 TO TYPE 2 CHANNELS IN MILLIVOLTS

It is only possible to access an active data file if DACØ4 is running concurrently. If an active file is not desired, simply strike a [CR] and enter the file name. If the file is on drive 0 , it must be specified (e.g., DX:DATA.DØB). If the extension is not specified, $\emptyset \emptyset A$ is assumed. Therefore, the entry, DATA, implies DX1:DATA.ØØA.

TABLE 10. Disk Block Data Produced by TYPAV

\begin{tabular}{|c|c|c|c|c|c|c|c|c|c|c|c|}
\hline $\begin{array}{r}947 \\
6 \\
30\end{array}$ & $\begin{array}{l}10 \\
60 \\
43\end{array}$ & $\begin{array}{l}1<0 \\
140\end{array}$ & $\begin{array}{l}150 \\
100\end{array}$ & $\begin{array}{l}220 \\
200\end{array}$ & $\begin{array}{l}230 \\
210\end{array}$ & $\begin{array}{l}240 \\
250\end{array}$ & $\begin{array}{l}166 \\
250\end{array}$ & 174 & 275 & 17 & 20 \\
\hline $\begin{array}{r}-3805 \\
5191 \\
4610 \\
-8192 \\
3450 \\
3609 \\
-4815 \\
-3831 \\
5191\end{array}$ & $\begin{array}{r}-3584 \\
3480 \\
3296 \\
3465 \\
3375 \\
-6192 \\
-2542 \\
-3583 \\
3479 \\
3289 \\
3466 \\
3366 \\
-8192 \\
-2523 \\
-3683 \\
3489 \\
3289 \\
3464 \\
3365\end{array}$ & $\begin{array}{r}3182 \\
7008 \\
3103 \\
3402 \\
3107 \\
-3840 \\
6194 \\
3182 \\
7005 \\
3102 \\
3402 \\
3089 \\
-3833 \\
6195 \\
3102 \\
7004 \\
3193 \\
3400 \\
3104\end{array}$ & $\begin{array}{r}4613 \\
-8192 \\
3450 \\
3360 \\
-4816 \\
-3833 \\
5192 \\
4666 \\
-8192 \\
3451 \\
3360 \\
-4827 \\
-3846 \\
5191 \\
4620 \\
-8192 \\
3449 \\
3358 \\
-4846\end{array}$ & $\begin{array}{r}3287 \\
3464 \\
3367 \\
-9132 \\
-2469 \\
-3584 \\
3479 \\
3287 \\
3464 \\
3375 \\
-8192 \\
-2535 \\
-3584 \\
3484 \\
3265 \\
3463 \\
3378 \\
-8192 \\
-2448\end{array}$ & $\begin{array}{r}3102 \\
3491 \\
3113 \\
-3843 \\
6194 \\
3184 \\
7005 \\
3103 \\
3400 \\
3108 \\
-3835 \\
6195 \\
3183 \\
7004 \\
3192 \\
3390 \\
3119 \\
-3855 \\
6194\end{array}$ & $\begin{array}{r}3450 \\
3358 \\
-4914 \\
-3849 \\
5190 \\
4699 \\
-8192 \\
3450 \\
3359 \\
-4893 \\
-3833 \\
5191 \\
4613 \\
-8192 \\
3450 \\
3358 \\
-4628 \\
-3849 \\
5131 \\
0\end{array}$ & $\begin{array}{r}3371 \\
-9192 \\
-2463 \\
-3584 \\
3480 \\
3298 \\
3463 \\
3354 \\
-6192 \\
-2549 \\
-3584 \\
3479 \\
3287 \\
3463 \\
3348 \\
-3192 \\
-2469 \\
-3583 \\
3400 \\
0\end{array}$ & $\begin{array}{r}3123 \\
-3856 \\
6194 \\
3181 \\
7005 \\
3164 \\
3480 \\
3106 \\
-3928 \\
6195 \\
3181 \\
7094 \\
3182 \\
3399 \\
3097 \\
-3845 \\
6195 \\
3182 \\
7005\end{array}$ & $\begin{array}{r}-4837 \\
-3844 \\
5190 \\
4698 \\
-8192 \\
3450 \\
3357 \\
-4831 \\
-3635 \\
3191 \\
4601 \\
-9192 \\
3445 \\
3357 \\
-4652 \\
-3954 \\
5191 \\
4627 \\
-8192 \\
0\end{array}$ & 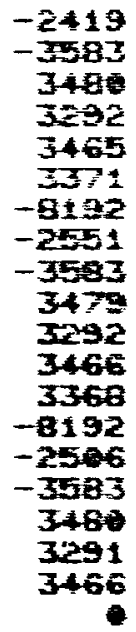 & 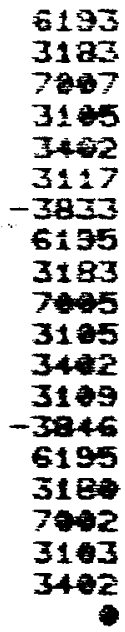 \\
\hline
\end{tabular}


As before, the number of channels/scan must agree with the value used in DACD4. A 1 is entered if the entire first block specified is desired in binary (i.e., the decimal equivalent of the binary number stored on disk). The first line gives the date ( 1 word) and time ( 2 words), respectively. The second and third lines are the channel/gain status codes in octal. (Note: gain code can change from initial value if auto gain option is selected in DACD4.) The remaining lines give the data. The values 8191 and -8192 represent +10 volts and -10 volts, respectively. With 21 channels/scan there is eleven blank spaces per disk block, which explains the zeroes on the last two lines. The same kind of data without any formatting could have been obtained by typing:

DUMP/TERM DATA. $\emptyset \emptyset[C R]$

without using TYPAV. The major advantage of using TYPAV to read disk blocks is the formatting of each channel/gain status word into bytes for ease of understanding. To continue with the entry:

$\underline{1[C R]}$

ENTER TWO CHANNELS

$\underline{\emptyset[C R]}$

$5[\mathrm{CR}]$

ENTER FIXED GAIN FOR FIRST CHANNEL SPECIFIED

[CR]

ENTER FIXED GAIN FOR SECOND CHANNEL SPECIFIED

500[CR]

where Table 11 shows a typical output.

TYPE 1 TO CONTINUE

The date code and start time is reported from the first block specified. Both data in millivolts and programmable gain (PRGN) are given for each channel selected. In addition, each data value is numbered consecutively and the disk block from which it was read is displayed. The headings DATA $\emptyset$, PRGN $\emptyset$, DATAT, and PRGN1 correspond to the symbols used in both GRAFAV and TYPAV. It is worth noting that the value, -294.113 millivolts corresponds to the value -3855 in the 
TABLE 11. Two Channel Data Produced by TYPAV

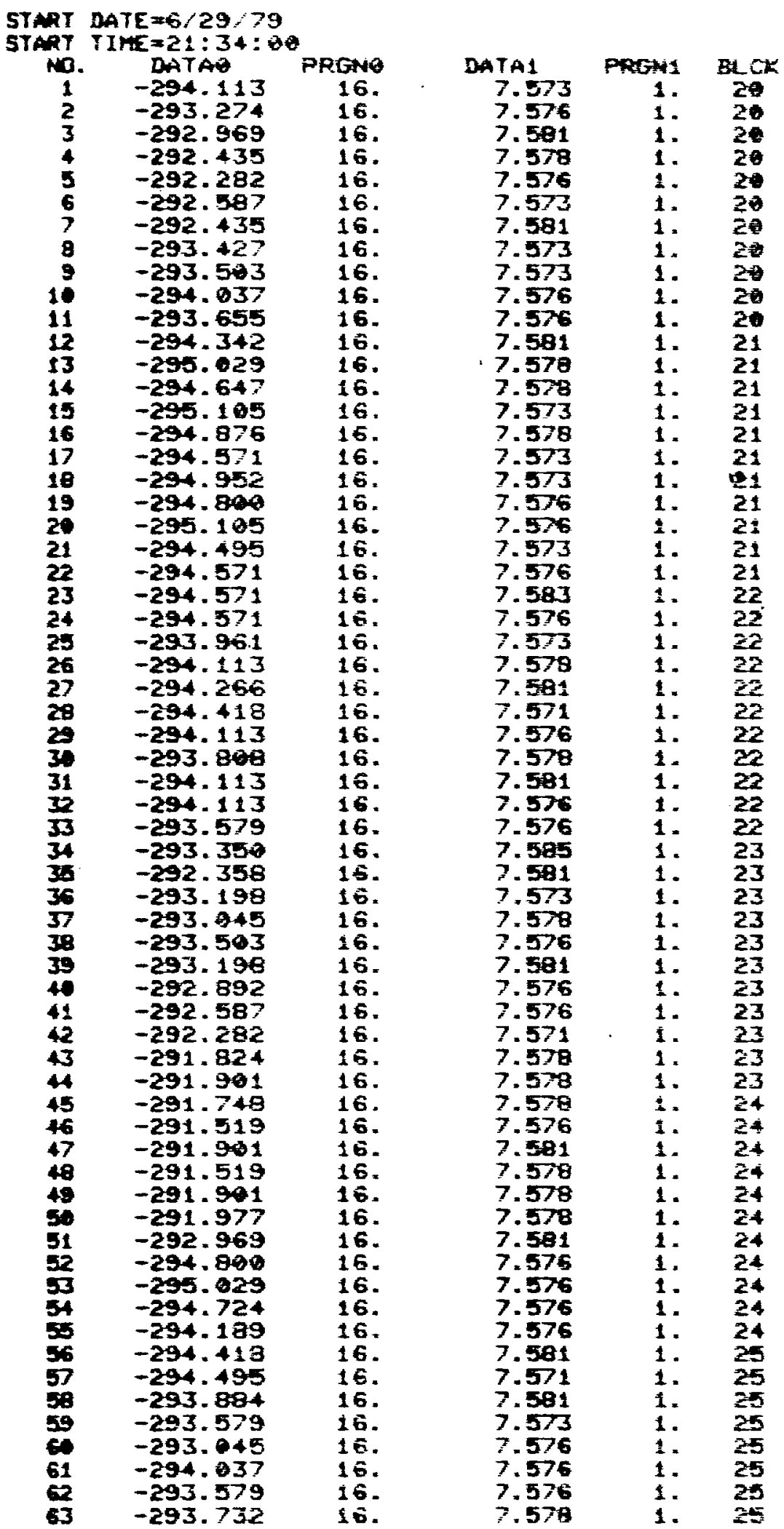


disk block data above [i.e., $-3855 \times 10000 /(8192 \times 16)]$. Likewise, the value, 7.573 corresponds to the 3102 (sixth entry from left in first data line) in the disk block data above. (Note that scan 0 and 5 were selected, which corresponds to the first and sixth data entry.) As is customary, the final message allows the routine to be continued without restarting it.

\subsection{TYPLOG OPERATION}

The program TYPLOG is a short FORTRAN program designed to run in background. A sample entry is given below assuming TYPLOG.SAV is resident on drive 1 .

CTRL/B

.RU TYPLOG [CR]

ENTER FILE NAME

*LOGQ1

ENTER 6 LINES OF TEXT DATA

A TANK SPARK OCCURRED AT [CR]

1653 WITH A RESULTING TEMPERATURE [CR]

TRANSIENT TO 325 C.... [CR]

RUN $24-3-5 / 14-J U N-79 \ldots[C R]$

[CR]

[CR]

TYPE 1 TO TYPE MORE DATA

[CR]

STOP--

Device DXI is implied and.TXT is the default extension (i.e., if not specified). Six lines of text data must be entered where the appropriate number of carriage returns may be used to complete the balance as shown above. Again, as shown, the user is given the option of entering more text data. Ordinarily the file name, DATA, should not be used unless it is intended to delete DATA.TXT created during initialization of DACD4. It is recommended that text data be written to the nonactive disk (i.e., the disk not in current use by 
DAC(4). Should the user attempt to create a text file on a properly squeezed active disk, the error message: "-2 = IENTER ERROR CODE," wi 17 be displayed, which means that the program was unable to find space to create a file. This happens because DAC $\emptyset 4$ reserves the largest continguous block of space on the active disk for itself.

\subsection{RETXT OPERATION}

The program RETXT is a short FORTRAN program run in background. It is a general purpose routine designed to read ASCII text data. It is coded, however, to read only block 0 of the specified file. Nevertheless, the code could be generalized in the future to read text data of any length. A typical example is given below:

$\underline{C T R L / B}$

.RU RETXT[CR]

ENTER FILE NAME

*LOGO][CR]

A TANK SPARK OCCURRED AT

1653 WITH A RESULTING TEMPERATURE

TRANSIENT TO 325 C....

RUN 24-3-5/14-JUN-79...

TYPE 1 TO READ MORE TEXT DATA

where the extension is assumed to be .TXT unless otherwise specified.

\subsection{REINT3 OPERATION}

REINT3 is a FORTRAN program designed specifically to read and display the initialization data file, DATA.INT, written by DAC $\varnothing 4$. An example of its operation is given below:

CTRL/B

.RU REINT3[CR]

ENTER FILE NAME: DEFAULT EXT $=$ INT

*DATTA[CR] 
Table 12 shows the typical output table which immediately follows the last entry above. (See Table 2 for a description of each entry in the table.) Since DACØ4 creates the file DATA. INT by simply writing a 256-word section of memory to disk, the user should exercise caution in modifying the data storage section in DAC $\varnothing 4$ between the variables SPAVAL and CHNSTA. Any changes in this section of MACRO code will likely create formatting problems in REINT3 and prevent the automatic restart feature of DAC 4 from functioning properly.

\subsection{RESET OPERATION}

Reset is a very short program which uses the PLOT55 routine to clear the graphic display. Simply type:

CTRL/B

.RU RESET[CR]

STOP.-

The program automatically terminates and control returns to the RT-11 monitor.

TABLE 12. Initialization Data Produced by REINT3

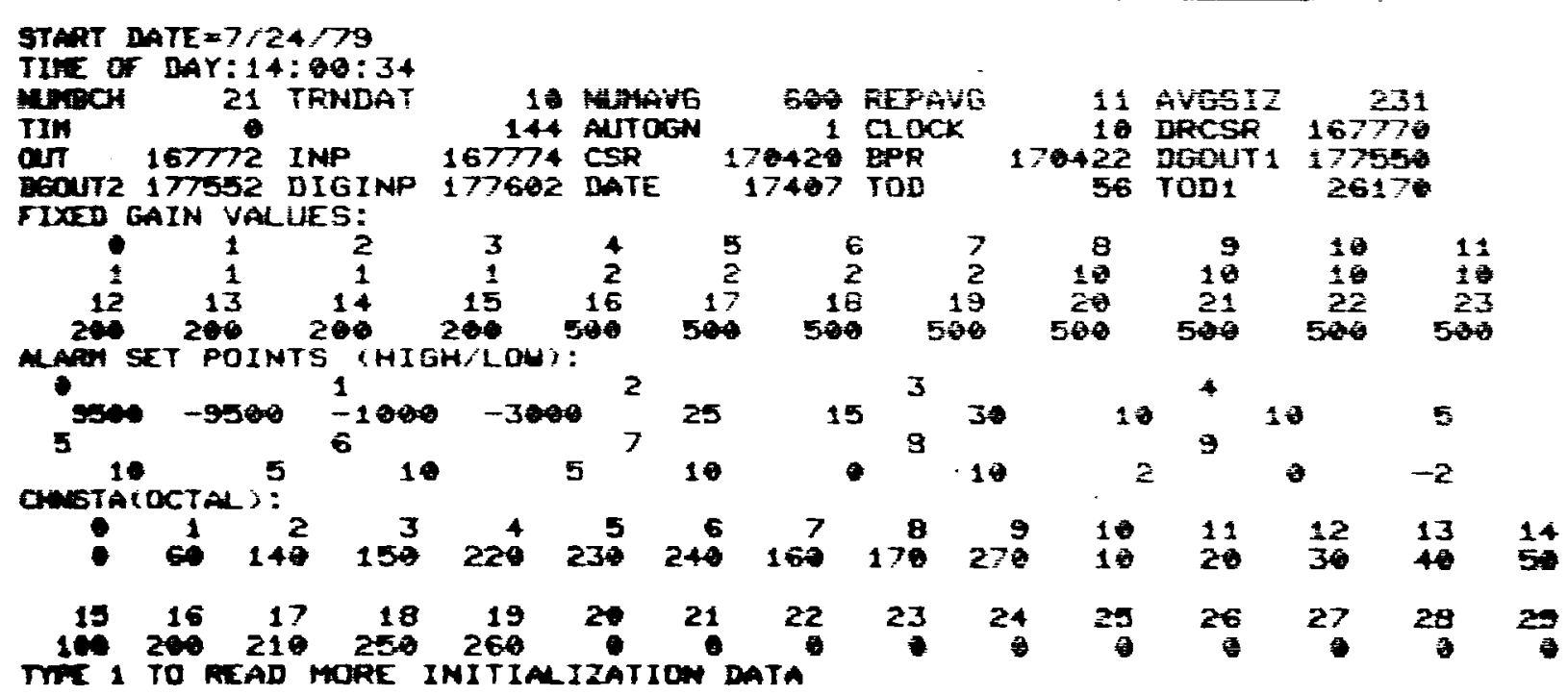




\section{APPENDIX A}

COMPUTER HARDWARE DESCRIPTION 
APPENDIX A

COMPUTER HARDWARE DESCRIPTION

\section{A. 1 COMPUTER CONFIGURATION}

The computer was orginally purchased in a stand-alone configuration frcm Digital Equipment Corporation (DEC) through an OEM supplier, First Computer corporation. It was purchased as a package, designated PDP11V/03, in a selfcontained, roll-around cabinet. However, several changes were stipulated in the purchase order that caused the package delivered to be somewhat nonstandard. The system as delivered included the components shown in Table A.l.

\section{TABLE A.1. Original DEC PDP11V/03 Components}

$\begin{array}{ll}\text { Model No. } & \text { CPU } \\ \text { KDI1-HA } & \text { 16K words memory } \\ \text { MSV11-DD } & \text { Expander box } \\ \text { BA11-ME } & \text { Rol1-around cabinet } \\ \text { H984 } & \text { Expansion cable with interface modules } \\ \text { BCV1B-06 } & \text { Programmable real-time clock } \\ \text { KWV11-A } & \text { Bootstrap module } \\ \text { REV11-A } & \text { Dual floppy disk and interface } \\ \text { RXV11-BA } & \text { Parallel 1ine interface } \\ \text { DRV11 } & \text { Graphics terminal with hardcopy } \\ \text { VT55-FA } & \text { Serial interface } \\ \text { DLV11 } & \text { EIS/FIS Micron Chip } \\ \text { KEV11 } & \end{array}$

Peripherals were subsequently interfaced to the computer in order to configure it as a self-contained Data Acquisition System. These peripherals are listed in Table A.2. 
TABLE A.2. Additional Components Added to PDP11V/03 Computer

\begin{tabular}{|c|c|c|}
\hline Model No. & Mfg & Description \\
\hline $620 / 100$ & NEFF & 24-channel analog-to-digital converter \\
\hline PM-SV32A & Plessey & $16 \mathrm{~K}$ words memory \\
\hline $1412 D A-4$ & ADAC & 4-channel digital-to-analog coverter \\
\hline $1632 \mathrm{HCO}$ & ADAC & 32-bit digital output interface \\
\hline $1616 \mathrm{CCI}$ & ADAC & 16-bit digital input interface \\
\hline DLV11-J & DEC & 4-channel serial interface \\
\hline$B N-52 A-7 F$ & DEC & $20 \mathrm{~mA}$ to EIA conversion kit \\
\hline BC 21B-05 & DEC & Cable \\
\hline BC $01 V-125$ & DEC & Cable \\
\hline BC $20 \mathrm{~N}-05$ & DEC & Cable \\
\hline
\end{tabular}

These additional components permitted the acquisition of analog and digital input, output of analog and digital signals, expansion of memory to the maximum allowable (i.e., 29k words), and the net addition of three serial interface channels for communication with existing peripherals and/or computers. The analog-to-digital converter can be expanded up to 64 analog input channels within the present $A / D$ chassis or to a total of 256 channels by the addition of three satellite chassis (i.e., 64 channels/chassis). Thus, considerable flexibility is obtained at a relatively low incremental cost. Likewise, additional peripherals can be added to the computer by using the necessary interface cards. The principal limitation is the number of available slots within the computer backplane. The backplane is designed to accept circuit boards having either two or four edge connectors. A board with two edge connectors is called a double-width board and measures approximately 132 by $228 \mathrm{~mm}(5.2$ by 9 in.). Likewise, a board with four edge connectors is called a quad-width board and measures approximately 267 by $228 \mathrm{~mm}$ (10.5 by 9 in.). Figure A.1 shows the schematic backplane as delivered to the University of Washington. As indicated, the programmable clock is the only board requiring a full quadwidth slot. Also shown in Figure A.1 are the two double-width slots used in connecting the main backplane box to the expansion backplane and the two spare double-width slots available for future expansion. Three of the four serial interface channels are also available (i.e., the first channel is used as the VT55 terminal interface). 


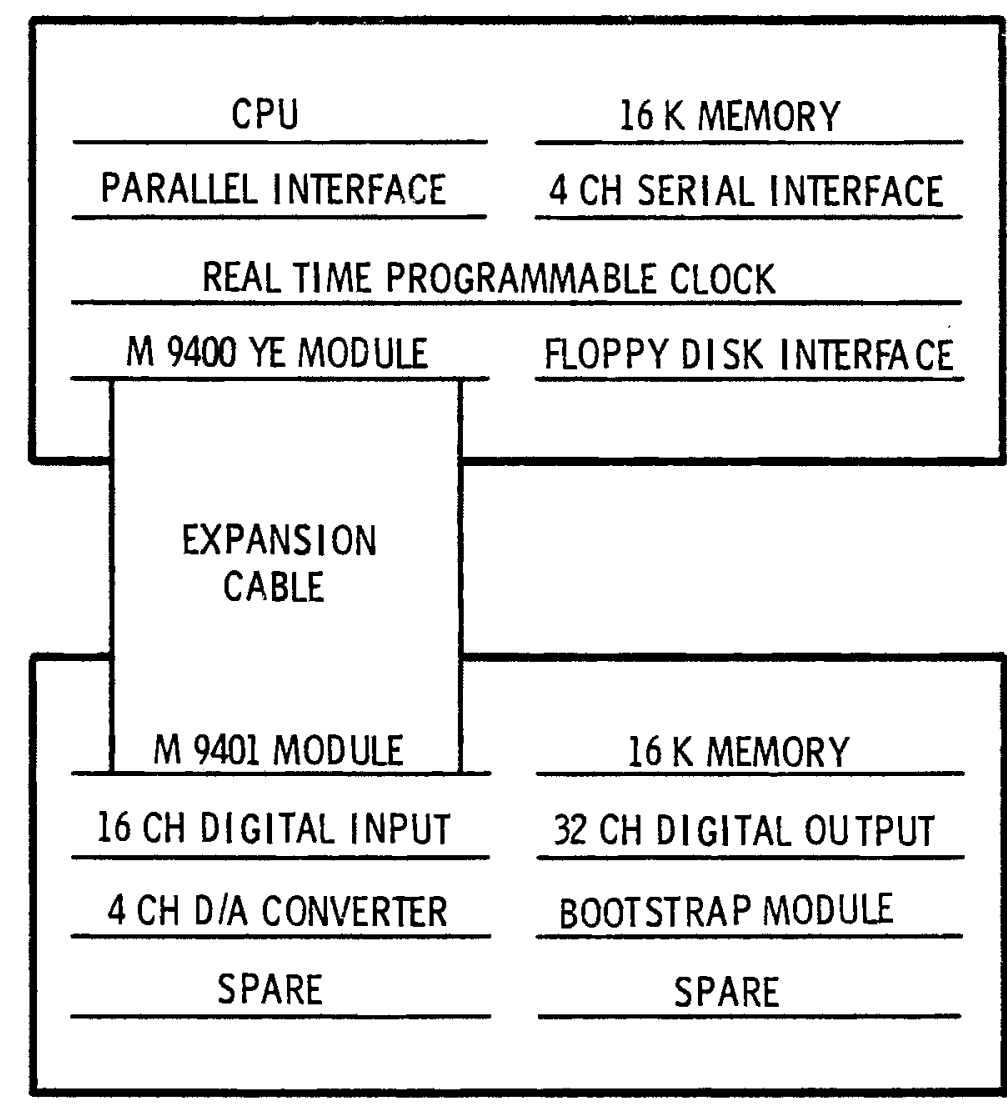

FIRST
BACKPLANE

FIGURE A.1. Schematic Backplane Configuration 


\section{A.2 ANALOG-TO-DIGITAL CONVERTER SUBSYSTEM}

The analog-to-digital (A/D) converter subsystem was procured from Neff Instrument Corporation. A summary of characteristics and specification is given in Table A.3.

\begin{tabular}{ll} 
TABLE A.3. Summary of Analog-to-Digital & Converter Specifications \\
\hline Model no. & $620 / 100$ \\
Maximum no. of channels & 256 \\
Low-level differential amplifiers & 12 \\
High-level differential amplifiers & 12 \\
Fixed gain range (low-level) & $10-1000$ \\
Fixed gain range (high-level) & $1-10$ \\
Options & $8-$ channel output buffer, \\
& control panel \\
Programmable gains & $1,2,4,8,16,32$ \\
Modes & Random/sequential \\
& remote/local \\
Interface & Programmed I/0 \\
ADC resolution & (DEC/DRV11) \\
(including sign) & 14 bits \\
Throughout rate & $20 \mathrm{kHz}$ \\
Full-scale input & $\pm 10 \mathrm{~V}$ \\
Overall system accuracy & $\pm 0.01 \%$
\end{tabular}

Detailed specifications can be found in the Neff Operation and Maintanence Manual. (4) The A/D Converter is of the amplifier-per-channel design with the inherent flexibility of changing fixed gain and filter cutoff for each channel. The system is delivered to the University of Washington with the settings indicated in Table A.4.

Both fixed gain and filter cutoff can be altered by changing plug-in modules on the appropriate printed circuit board. Spares have been procured and include twelve $10-\mathrm{Hz}$ filters, two x5-gain modules, and two x100-gain modules plus an extender card kit for maintenance. 
TABLE A.4. Summary of Fixed Gains and Filter Cutoff Frequencies

\begin{tabular}{|c|c|c|c|c|}
\hline Channel & Type ${ }^{(a)}$ & $\begin{array}{l}\text { Card } \\
\text { No. }\end{array}$ & $\begin{array}{l}\text { Fixed } \\
\text { Gain } \\
\end{array}$ & $\begin{array}{c}\text { Filter } \\
\text { Cutoff, } \\
\mathrm{Hz}\end{array}$ \\
\hline 0 & $H / L$ & 0 & 1 & 100 \\
\hline 1 & $H / L$ & 0 & 1 & 100 \\
\hline 2 & $\mathrm{H} / \mathrm{I}$ & 0 & 1 & 100 \\
\hline 3 & $H / L$ & 0 & 1 & 100. \\
\hline 4 & $H / L$ & 1 & 2 & 100 \\
\hline 5 & $H / L$ & 1 & 2 & 100 \\
\hline 6 & $H / L$ & 1 & 2 & 100 \\
\hline 7 & $H / L$ & 1 & 2 & 100 \\
\hline 8 & $H / L$ & 2 & 10 & 100 \\
\hline 9 & $H / L$ & 2 & 10 & 100 \\
\hline 10 & $H / L$ & 2 & 10 & 100 \\
\hline 11 & $H / L$ & 2 & 10 & 100 \\
\hline 12 & $L / L$ & 3 & 200 & 100 \\
\hline 13 & $L / L$ & 3 & 200 & 100 \\
\hline 14 & $L / L$ & 3 & 200 & 100 \\
\hline 15 & $L / L$ & 3 & 200 & 100 \\
\hline 16 & $L / L$ & 4 & 500 & 100 \\
\hline 17 & $L / L$ & 4 & 500 & 100 \\
\hline 18 & $L / L$ & 4 & 500 & 100 \\
\hline 19 & $L / L$ & 4 & 500 & 100 \\
\hline 20 & $L / L$ & 5 & 500 & 100 \\
\hline 21 & $L / L$ & 5 & 500 & 100 \\
\hline 22 & $L / L$ & 5 & 500 & 100 \\
\hline 23 & $L / L$ & 5 & 500 & 100 \\
\hline
\end{tabular}

(a) $H / L=$ high level; $L / L=$ low level

There are three locations on the I/O page of memory that are used by the DRV11 parallel interface for communications and control of the A/D converter. They are listed in Table A.5, as follows: 
TABLE A.5. Analog-to-Digital Converter Addresses

\begin{tabular}{|c|c|c|}
\hline Address & Symbol & Use \\
\hline 167770 & DRCSR & DRV11 Control Status Register where: \\
\hline & & $\begin{array}{l}\text { bit } \emptyset=\operatorname{CSR} \emptyset \text { (sequential/random) }(a) \\
\text { bit } 1=\text { CSR1 (not used) } \\
\text { bit } 5=\text { INT ENB B (not used) } \\
\text { bit } 6=\text { INT ENB A (not used) } \\
\text { bit } 7=\text { REQ A (ready/busy)(a) } \\
\text { bit } 15=\text { REQ B (remote/loca } 1)(a)\end{array}$ \\
\hline 167772 & OUT & Output Data Register (or ADC input) \\
\hline 167774 & INP & Input Data Register (or ADC output) \\
\hline
\end{tabular}

(a) The convention for describing the function of digital lines is as follows: high state/low state. For example, a high state on the sequential/random line selects sequential mode; a low state selects random mode. As with all TTL logic, an open line is held in the high state. Therefore, with the sequential/random 1 ine open, the system operates in sequential mode. (4)

As mentioned, the Neff analog-to-digital converter is interfaced to the computer via a DRVIl general purpose 16-bit parallel interface. A schematic of the interface connection is given in Figure A.2.

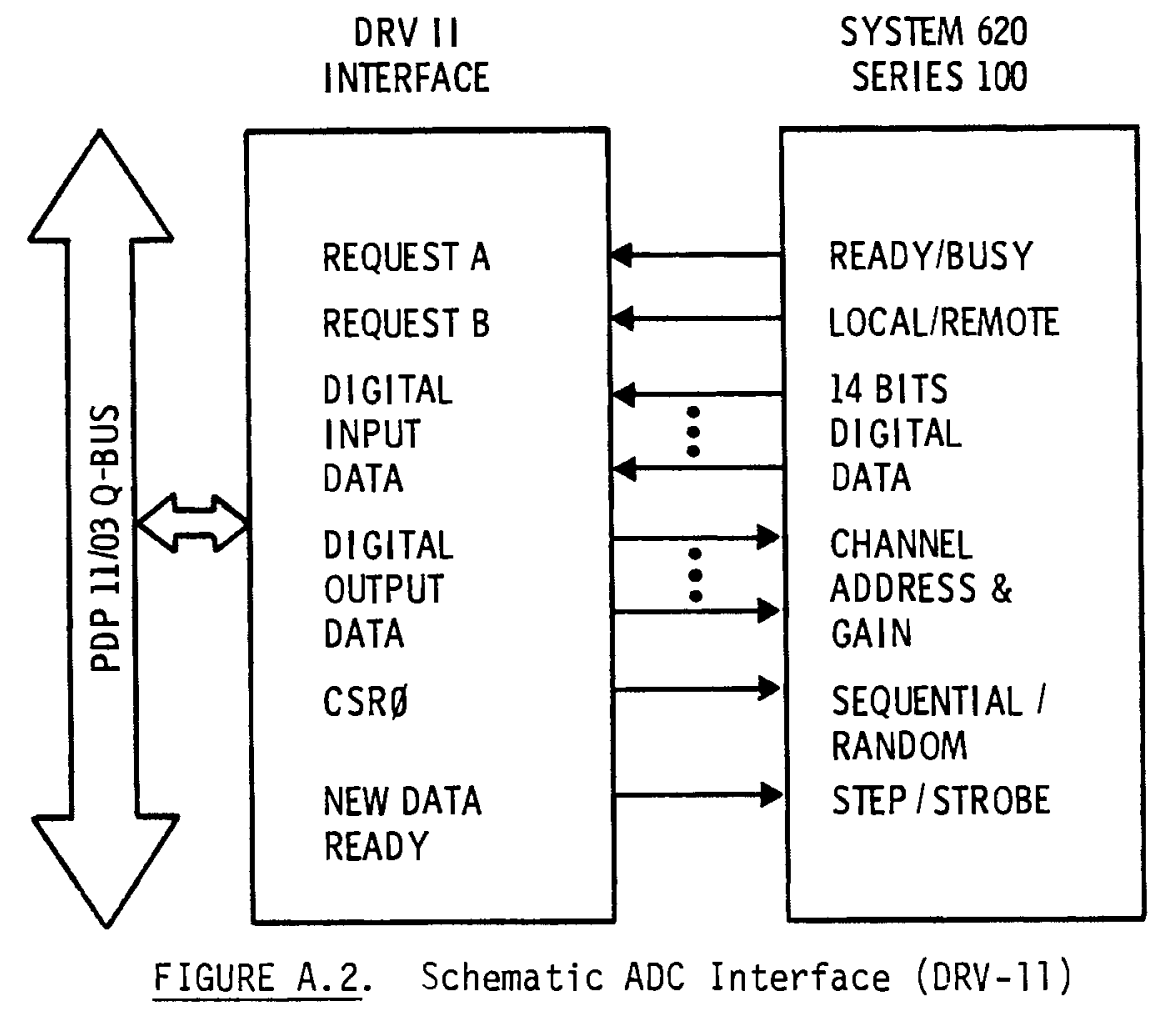


Both the Neff manual ${ }^{(4)}$ and the DEC Memories and Peripherals Handbook ${ }^{(5)}$ should be consulted for more details. Since the computer is a 16-bit machine and the analog-to digital converter is 14 bits, bit 13 (i.e., the sign bit) of the ADC data word has been connected to bits 13, 14, and 15 of the DRV11 data input word. This particular connection causes negative numbers generated by the analog-to-digital converter to be correctly interpreted by the computer in 2 's complement format without the necessity of performing any special software bit manipulation.

\section{A.3 DIGITAL OUTPUTS}

An ADAC model 1632HCO 32-channel digital output card was procured to interface to the computer. These outputs, in principle, could be used to actuate the beam stop, helium circulating pump, alarms, etc. Initially, the interface has been used to operate two 16-channel alarm panels, each consisting of 16 LEDs and one alarm speaker. Each panel is equipped with a test switch to verify proper operation of the LEDs. At present, lights 0 and 1 of digital output panel No. 1 are used to signify operation of disk drive 0 and 1 , respectively. This feature applies only to DAC $\varnothing 4$ operation, however. Also digital channels 0 through 9 of digital output panel No. 2 have been programmed to alarm when scans 0 through 9, respectively, reach either their highor low-level alarm set point. The audio alarm is designed to actuate on any change in status of the LEDS (i.e., when a light is either turned on or off). A description of the digital output card is given in the ADAC manual. (6)

\section{A.4 DIGITAL INPUTS}

An ADAC model 1616CCI 16-channel digital input card was procured to interface with the computer. In principle, it could be used to check the status of the beam stop, helium circulating pump, switch positions, etc. Initially, the interface has been connected to a 16-channel switch panel which allows the user to input and/or change parameters (e.g., during real-time data acquisition). The switch panel is mounted on a 48-cm (19-in.) relay chassis wirich also contains the analog input terminations for the ADC, the digital-to-analog output 
terminations, and a series of eight banana plug jacks for the ADC output buffers. A description of the digital input card is given in the ADAC manual. (7) Table 8 describes the present use of bits 0-7 of the digital input interface.

\section{A.5 DIGITAL-TO-ANALOG CONVERTER}

An ADAC model 1412DA-A four-channel digital-to-analog converter card was procured. The card is capable of either current or voltage proportioning controt on each channel. The interface is capable of replacing up to four analog temperature controllers if desired. There is an incentive to replace the specimen temperature controller due to its response characteristics. The ultimate decision will probably be based upon the reliability of the computer and compromises associated with the additional CPU overhead incurred (i.e., the temperature of the specimen would most likely have to be controlled inside the clock interrupt service routine in order to improve the response behavior). Details on the operation of the digital-to-analog converter can be found in the manual supplied by ADAC Corporation. (8) 
APPENDIX B

COMPUTER SOURCE CODE LISTINGS 


\author{
APPENDIX B \\ COMPUTER SOURCE CODE LISTINGS
}

\title{
B.7. DACФ4 MAC LISTING
}

TrPE DACF4. MAC

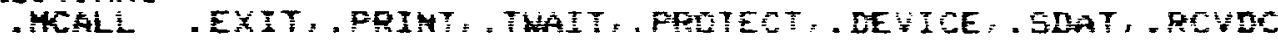

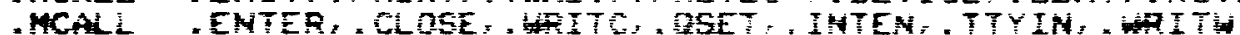

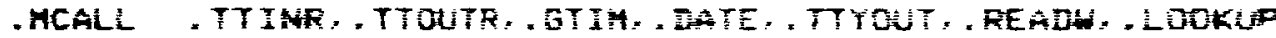

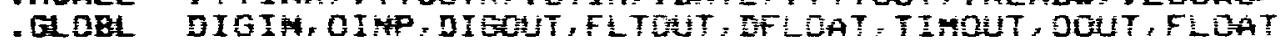

$15: 1=+4$

ERRBYT $=52$

TITLE DACQ4

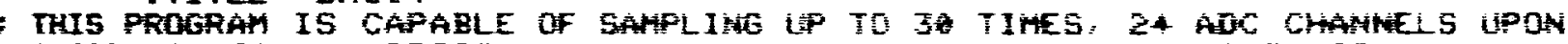

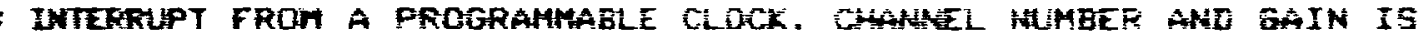

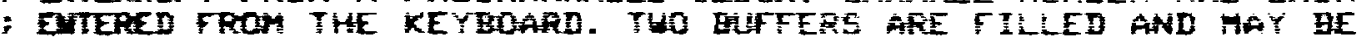

- AEAD FROM A BACKTROUMT PRCERAM: A TRANSTENT BLFFER AHD AN

; AVERAED BLFFER. THE AVERAGED BDEFER IS HPITTEN TO DISK.

; AUTOATIC GA

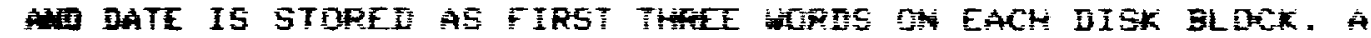

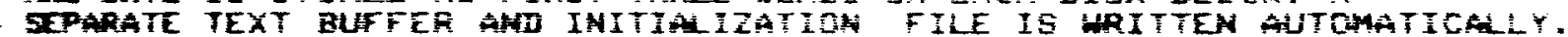

THIS VERSION DISPLAYS DATA SAIN SUWHA GH SCREEM UPON REDUEST:

THS VERSION ADDS TAELE OF FTKET GATHS FOP STCFAGE IM DATA. INT

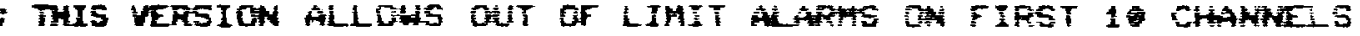

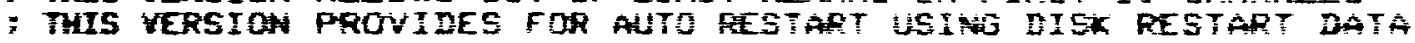

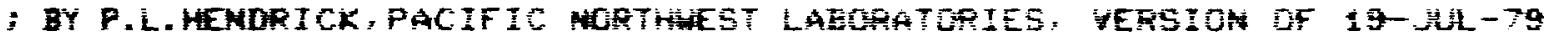

DACB?: TST RERCSF

EMI RETETE

PRINT ESEA

EXIT

FUTE: PRINT MHG12

PRTNT *HSEO

DEVICE APEA HSAVE

-OSET BA. 16

MOV FAREA, $-S$ S:

NON $+4,0, F 5$

PROTECT CSP), RE

ACC

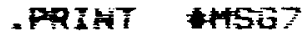

EXIT

Thent:

HOV ACLKIN, IRE

NOV H+3. CRE

-PRINTT ALSTZE

ISR R5, DISI

GP 1 , RQ

BaE SKIPA5

SWP RESTAF

SIP15: .PRINT HSOIS

JSR AS DIGEN

:TEST FER REMOTE STATLS OF ADC

FFit AIE IN FEMOTE

C $320, R$,

BNI SKIP15

MON RO, CLDCK

MLL $\quad 160.596$

HOV RI, Ro

CON RO

mov Ro, Sicki

; PATE E TIHE FENIUTER

IFTER GTRLF

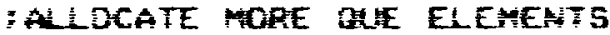

POOTEST GECK INTERRIFY VECTORS

FFUTECT EFPOA

:ESTABIISH INTEPALPT VECTOES

FESTART OPTICA SEIEST

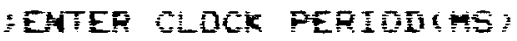

STDTE CLOCK IN 45

; SET CLOKE INTEPALPT PERIOD 


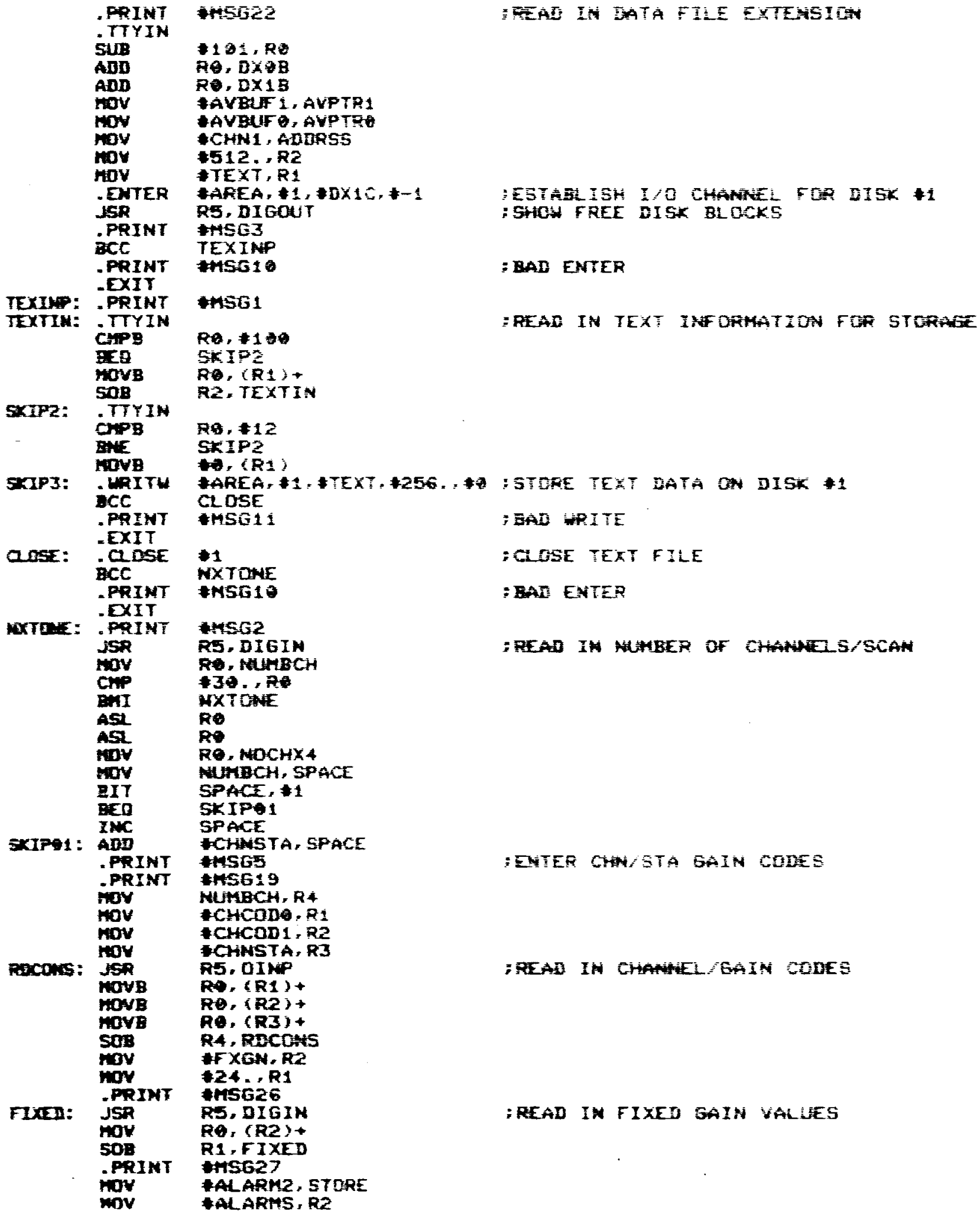




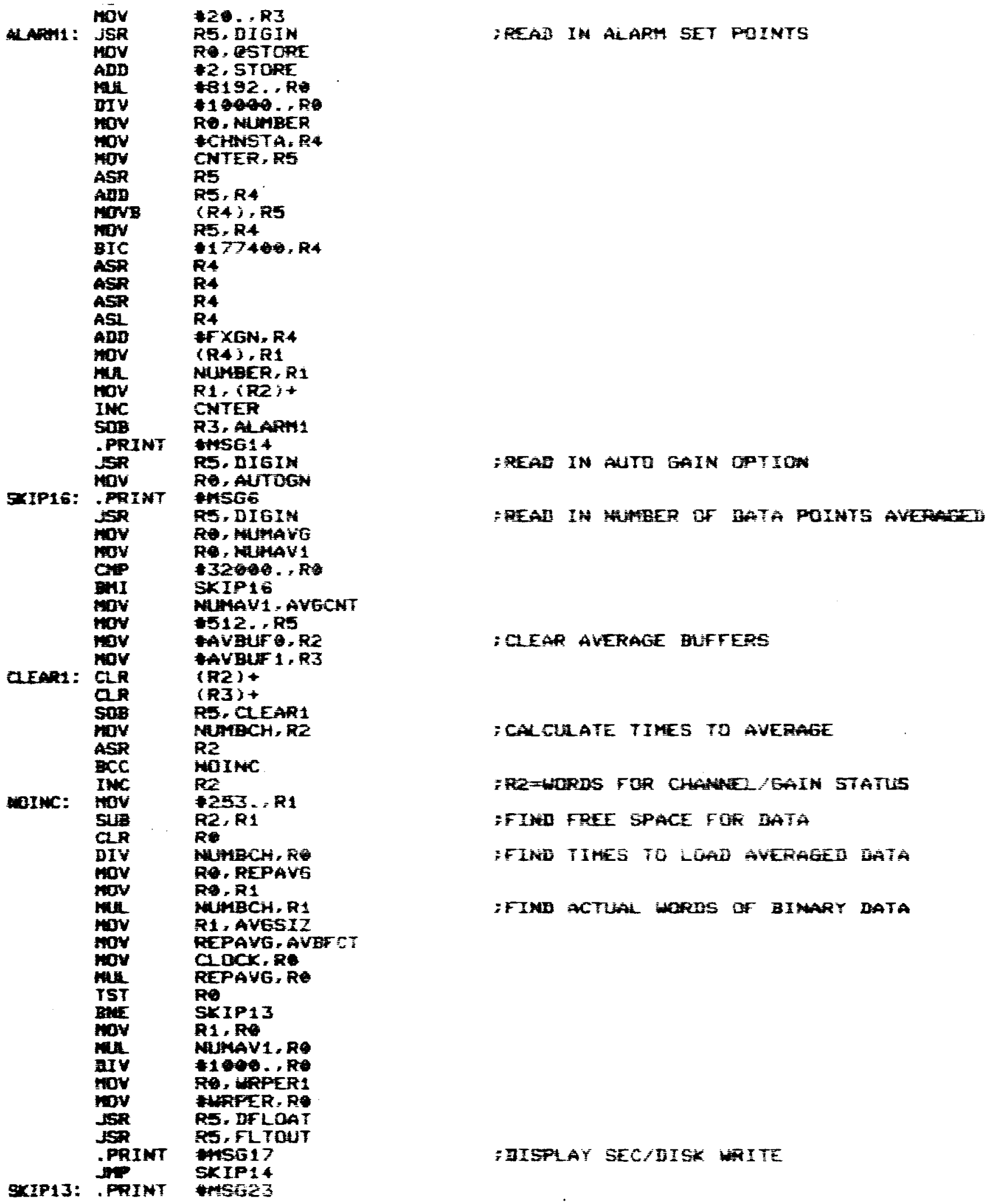




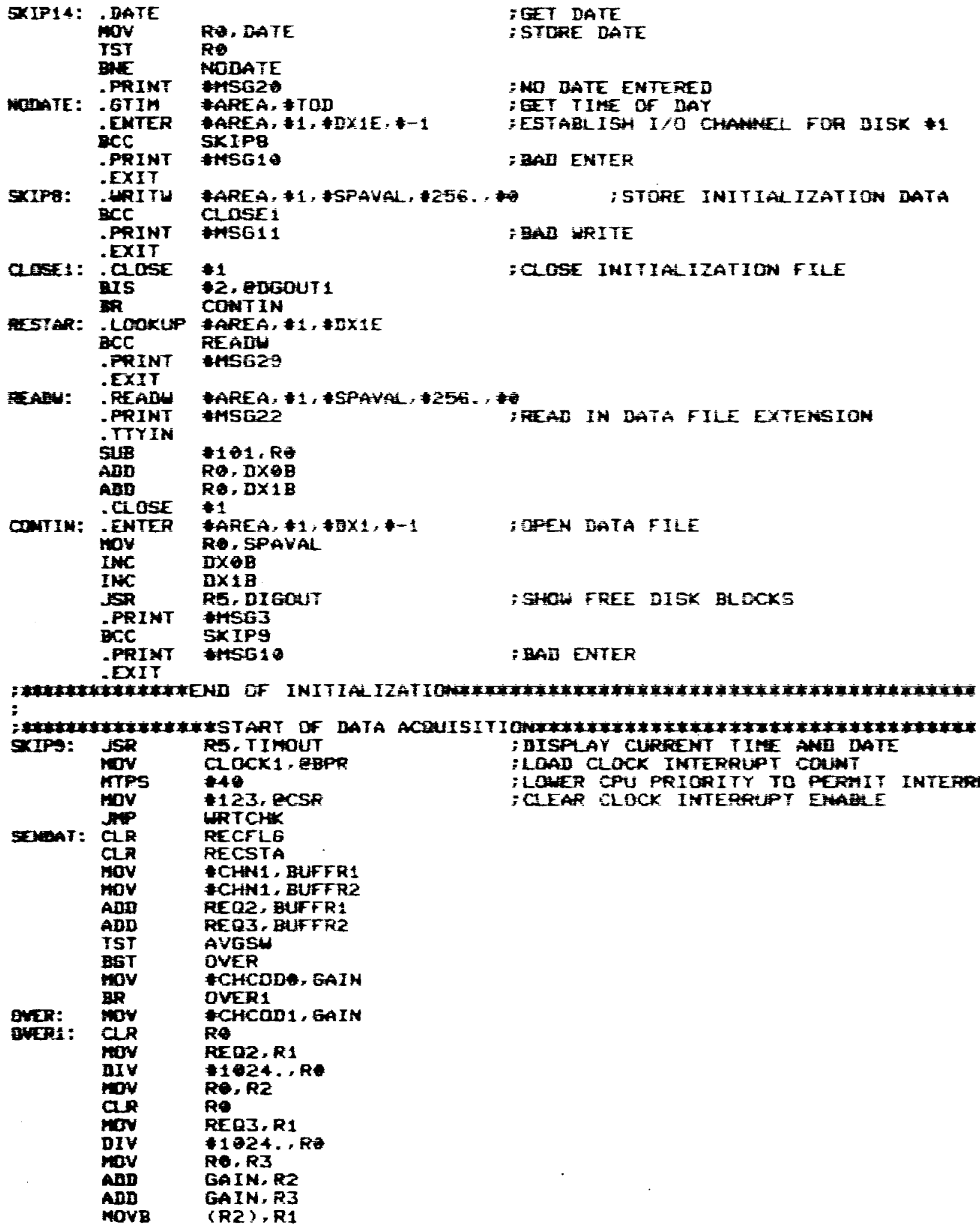




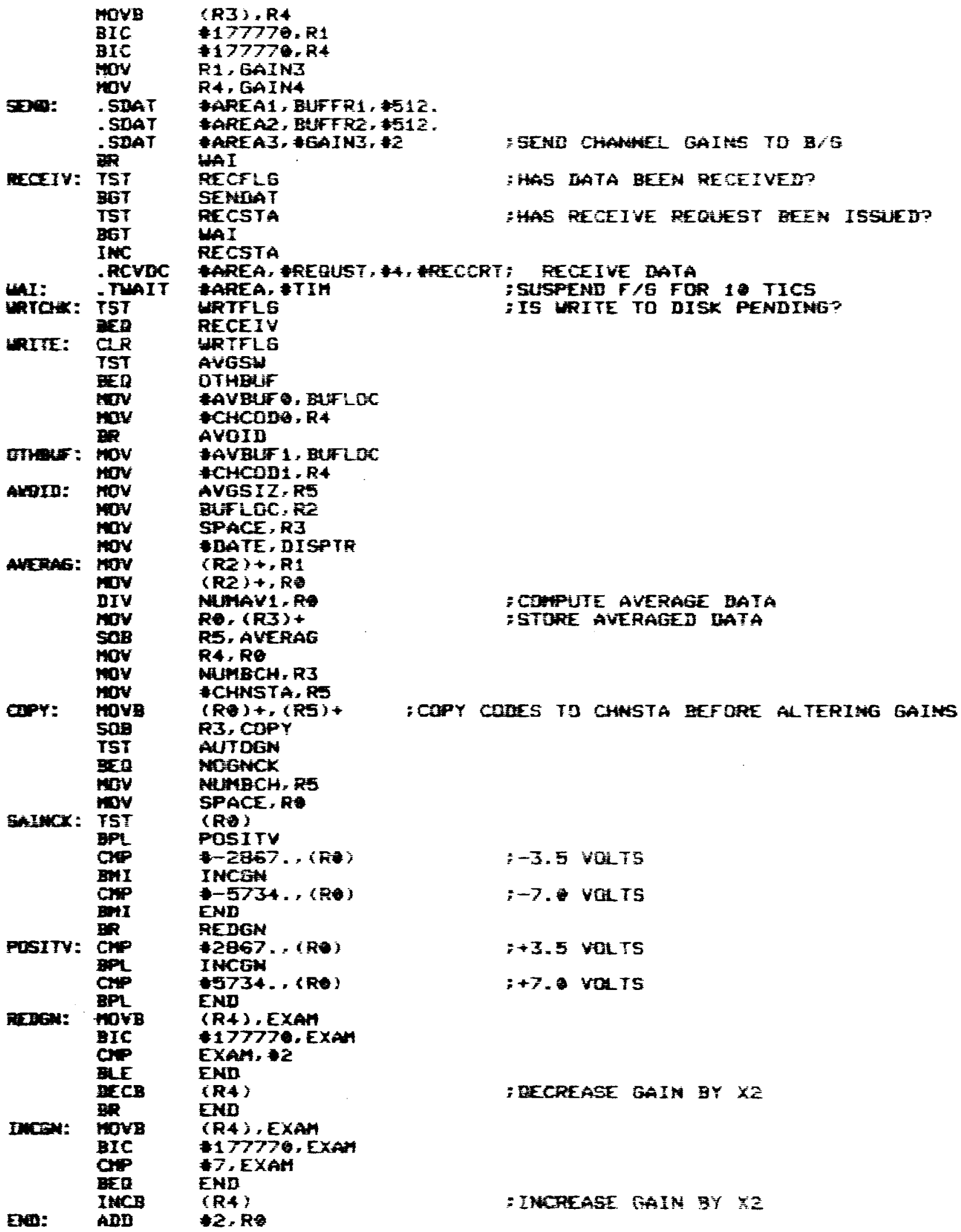




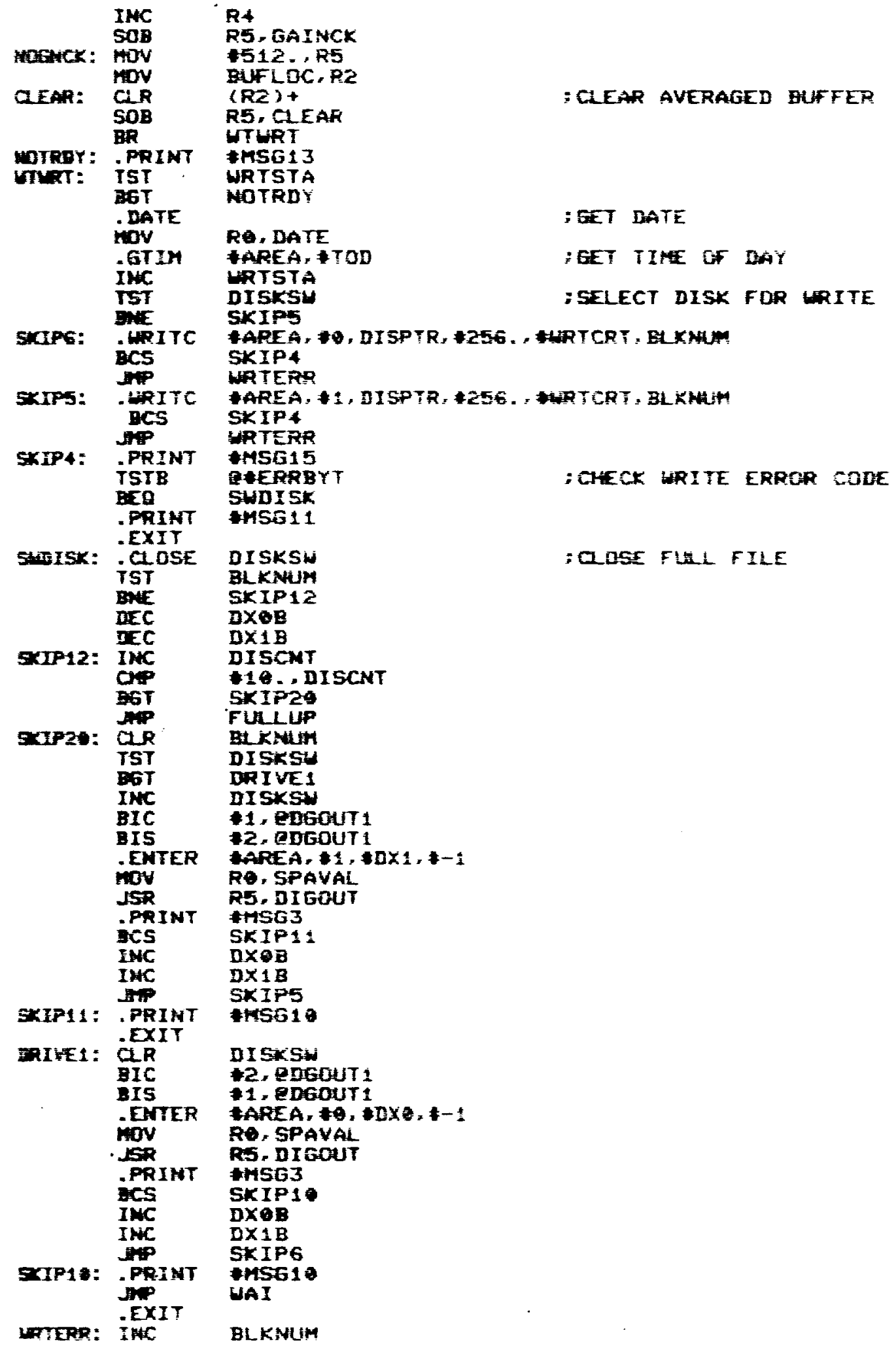




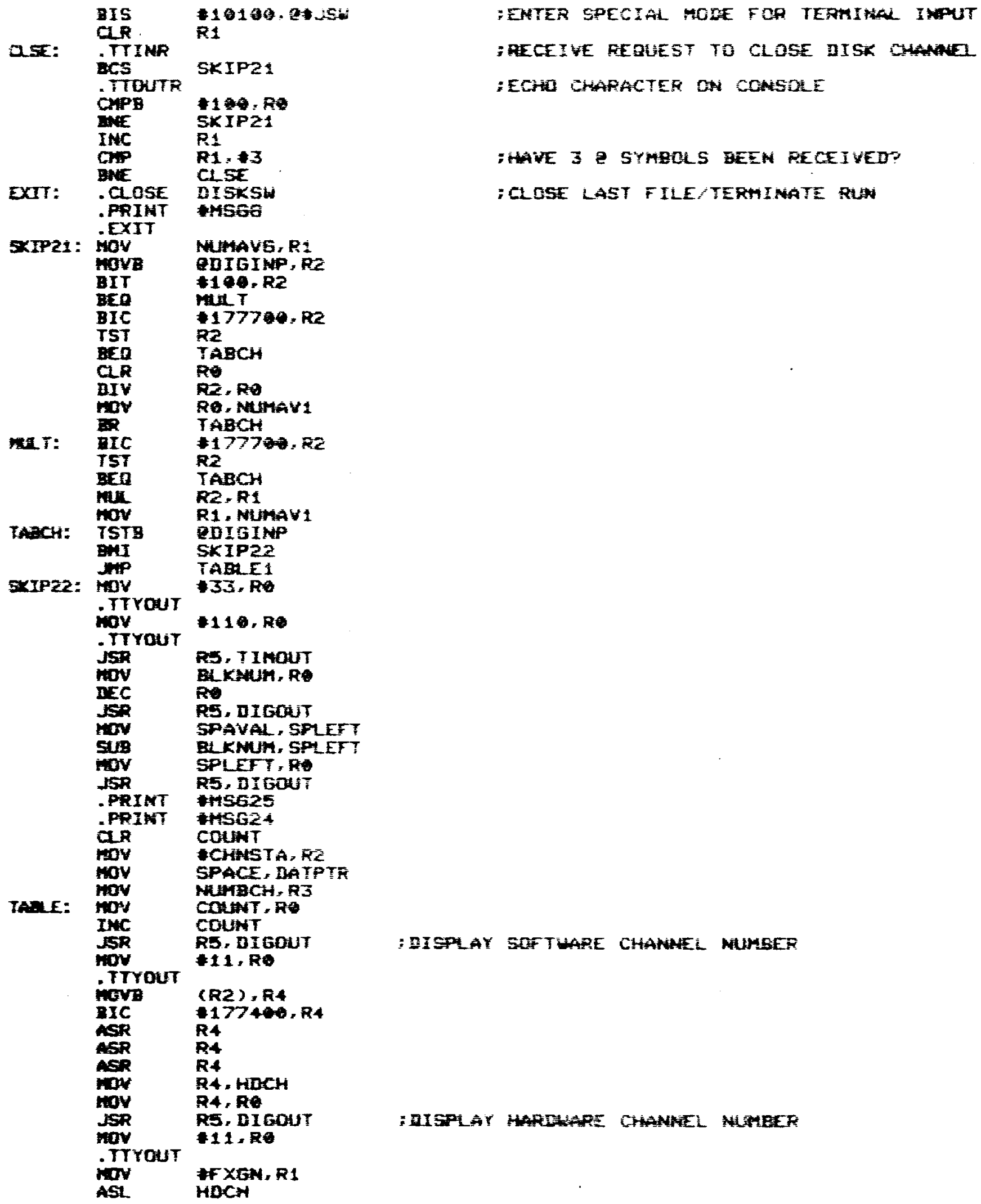




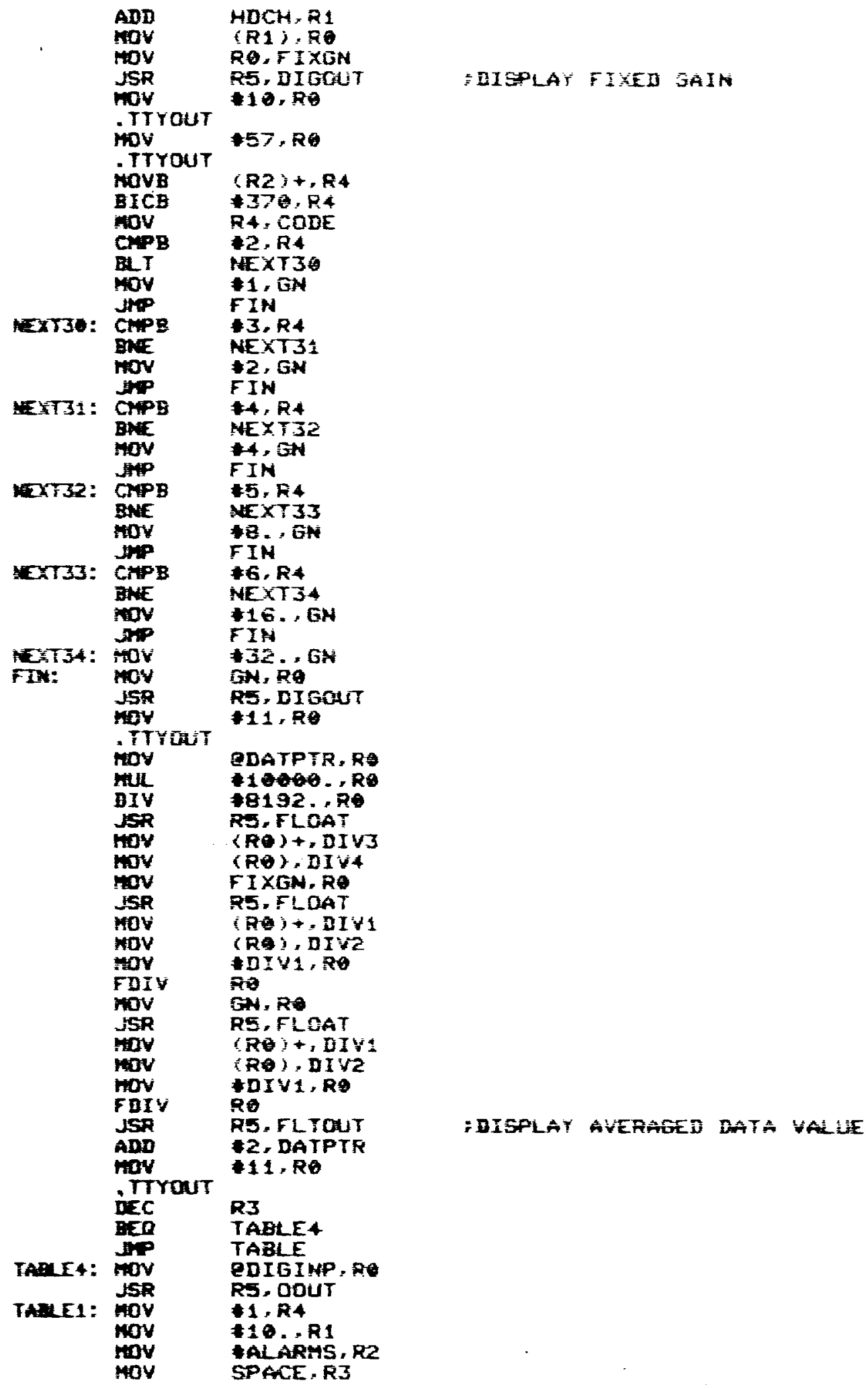




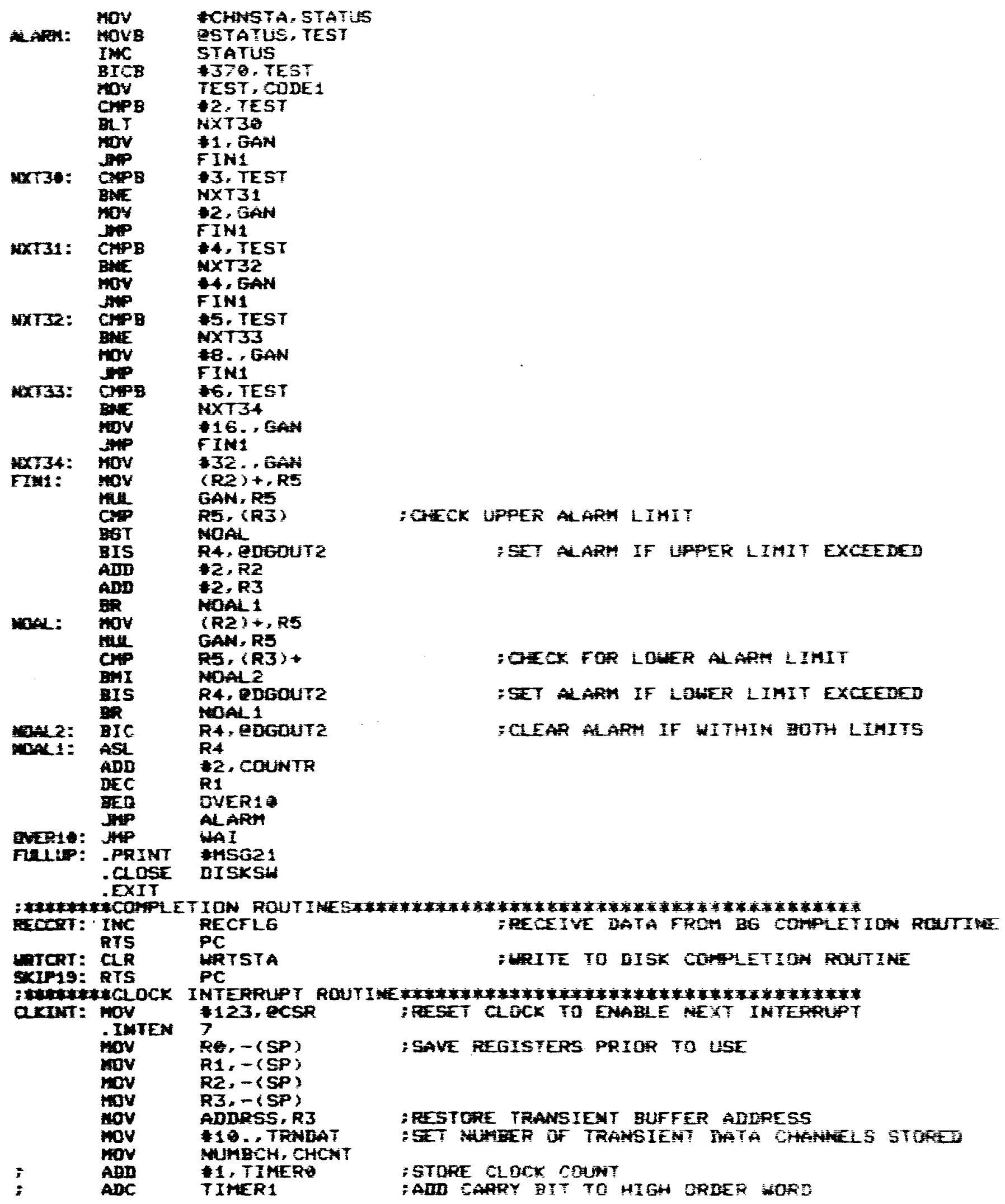




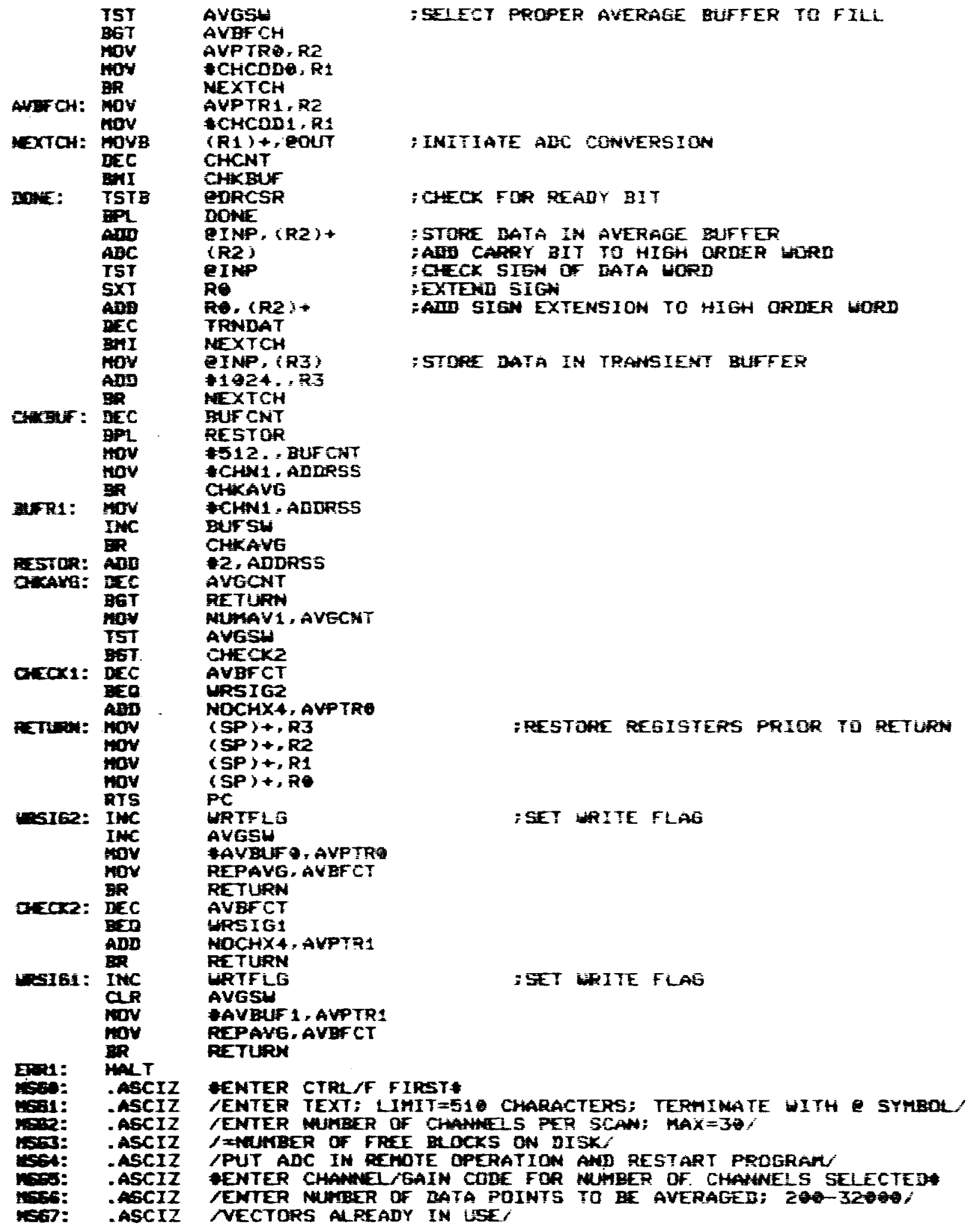




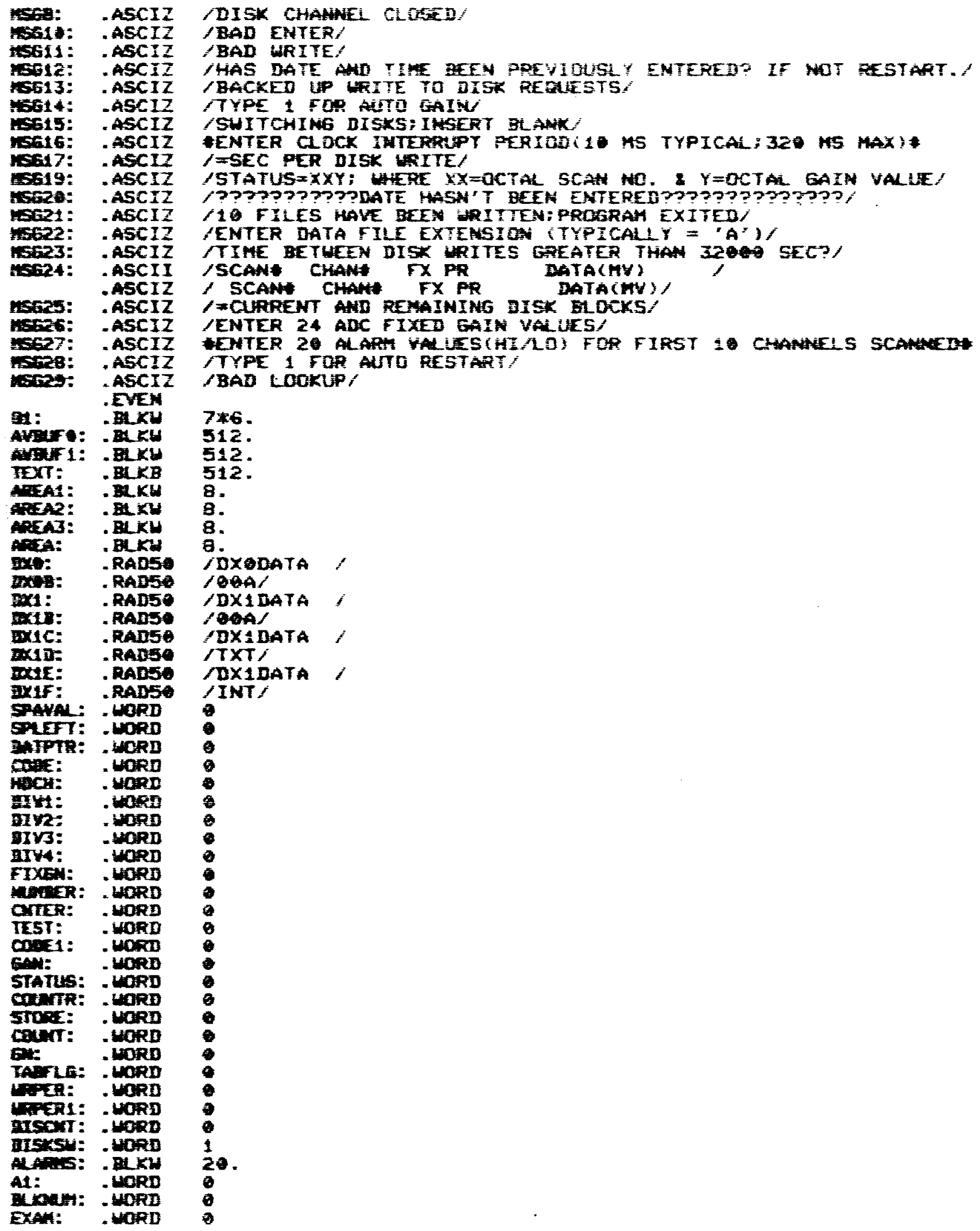




\begin{tabular}{|c|c|c|}
\hline 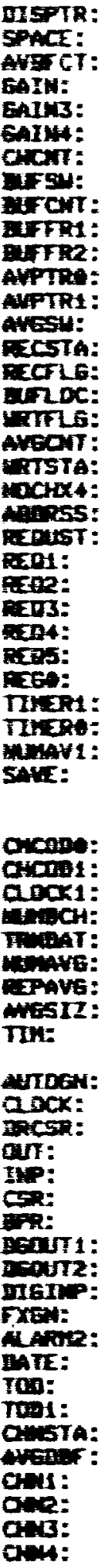 & 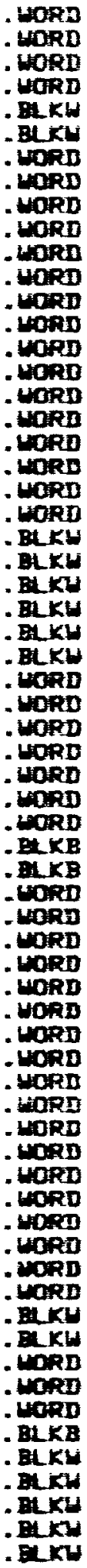 & 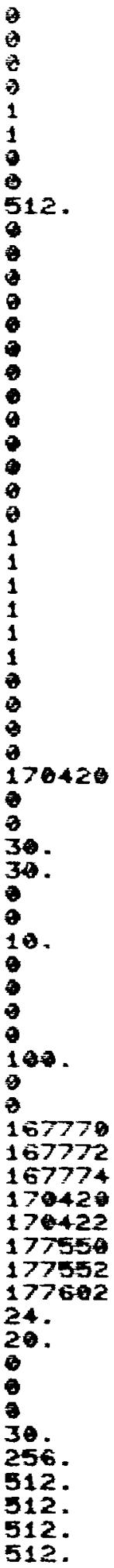 \\
\hline
\end{tabular}




\begin{tabular}{|c|c|}
\hline $\begin{array}{l}\text { cus: } \\
\text { ans: } \\
\text { anp: } \\
\text { anb: } \\
\text { gros: } \\
\text { cints: }\end{array}$ & 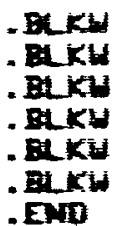 \\
\hline
\end{tabular}

\section{B.2 GRAFTR.FOR LISTING}

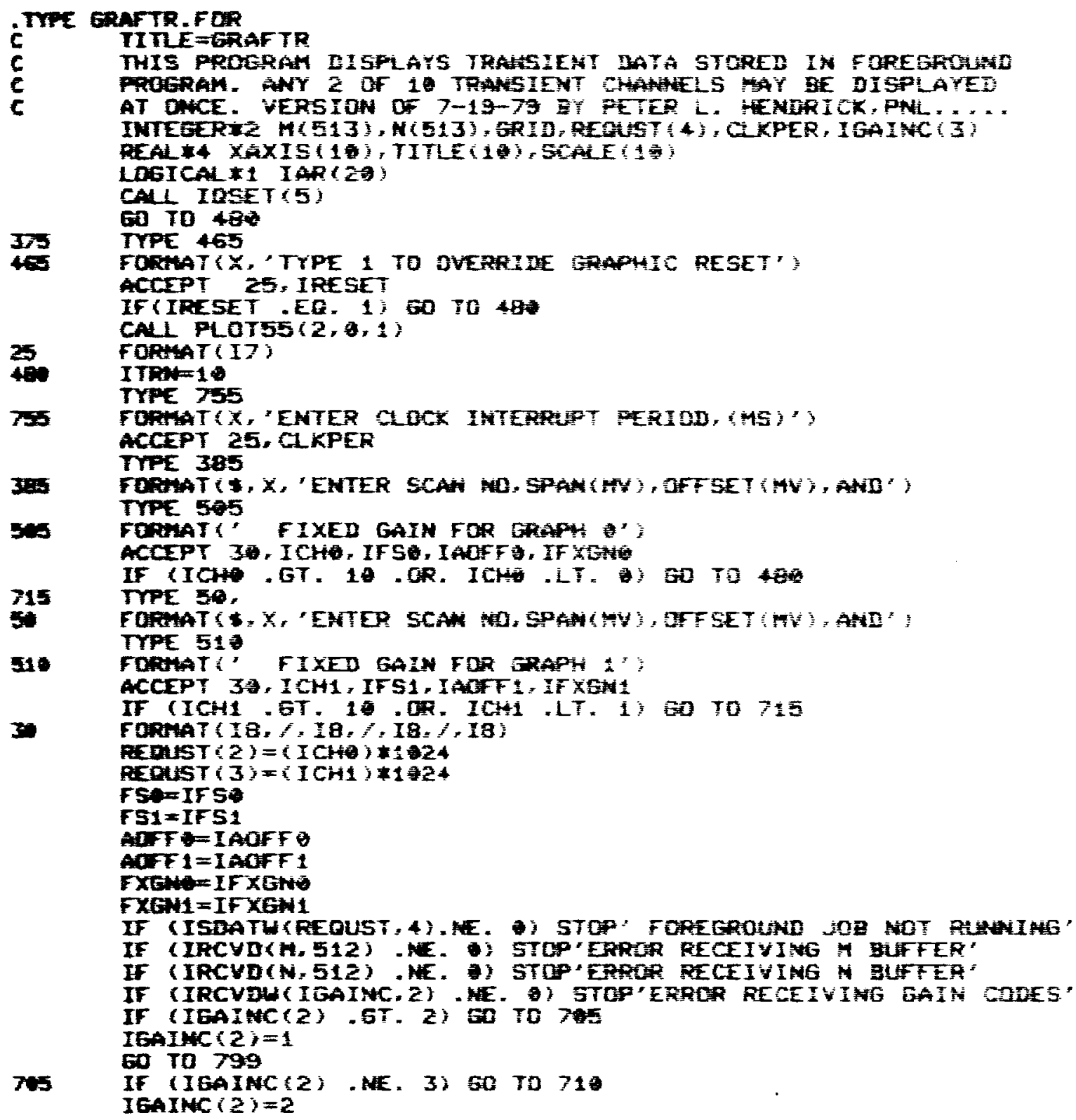




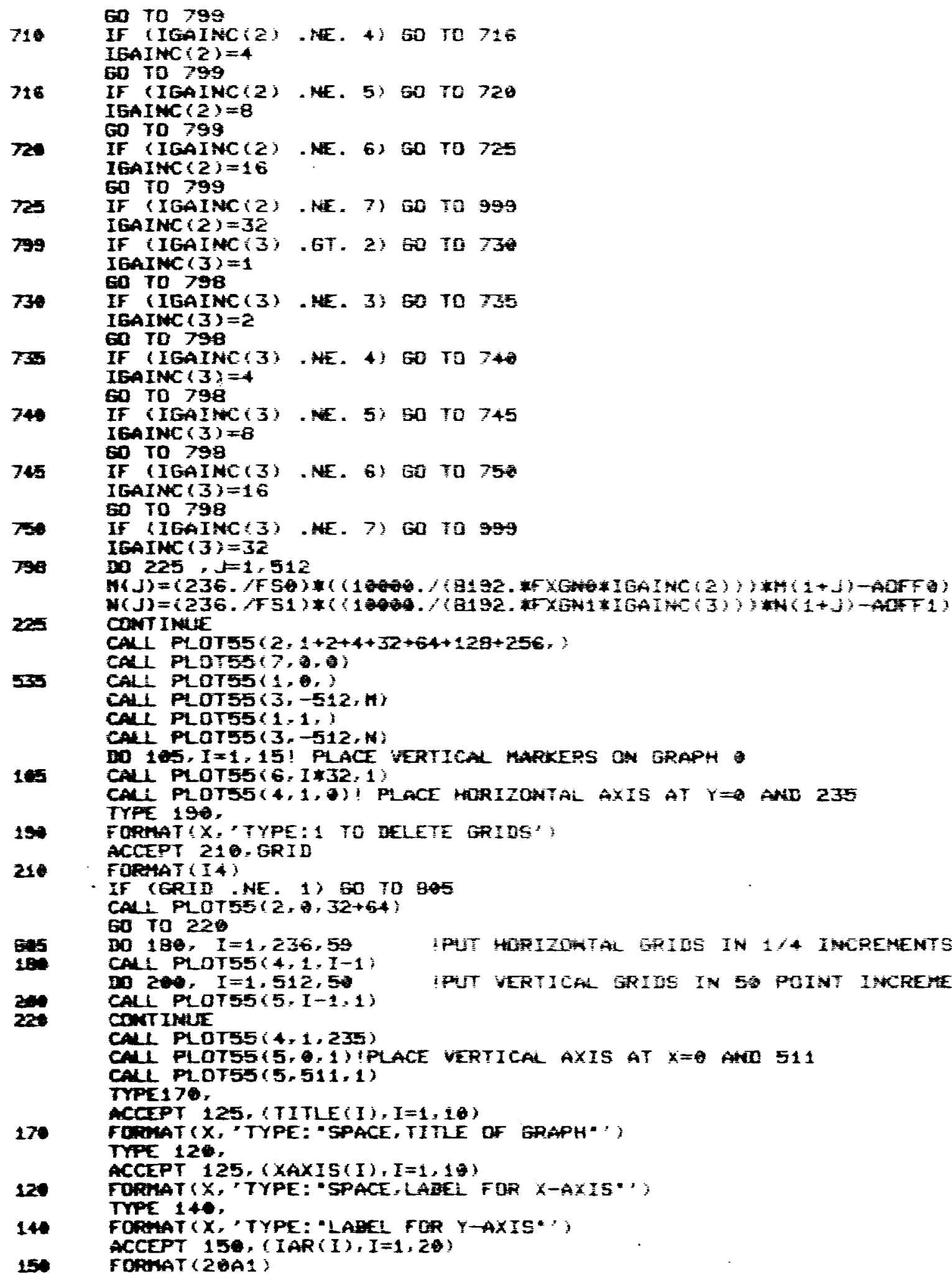




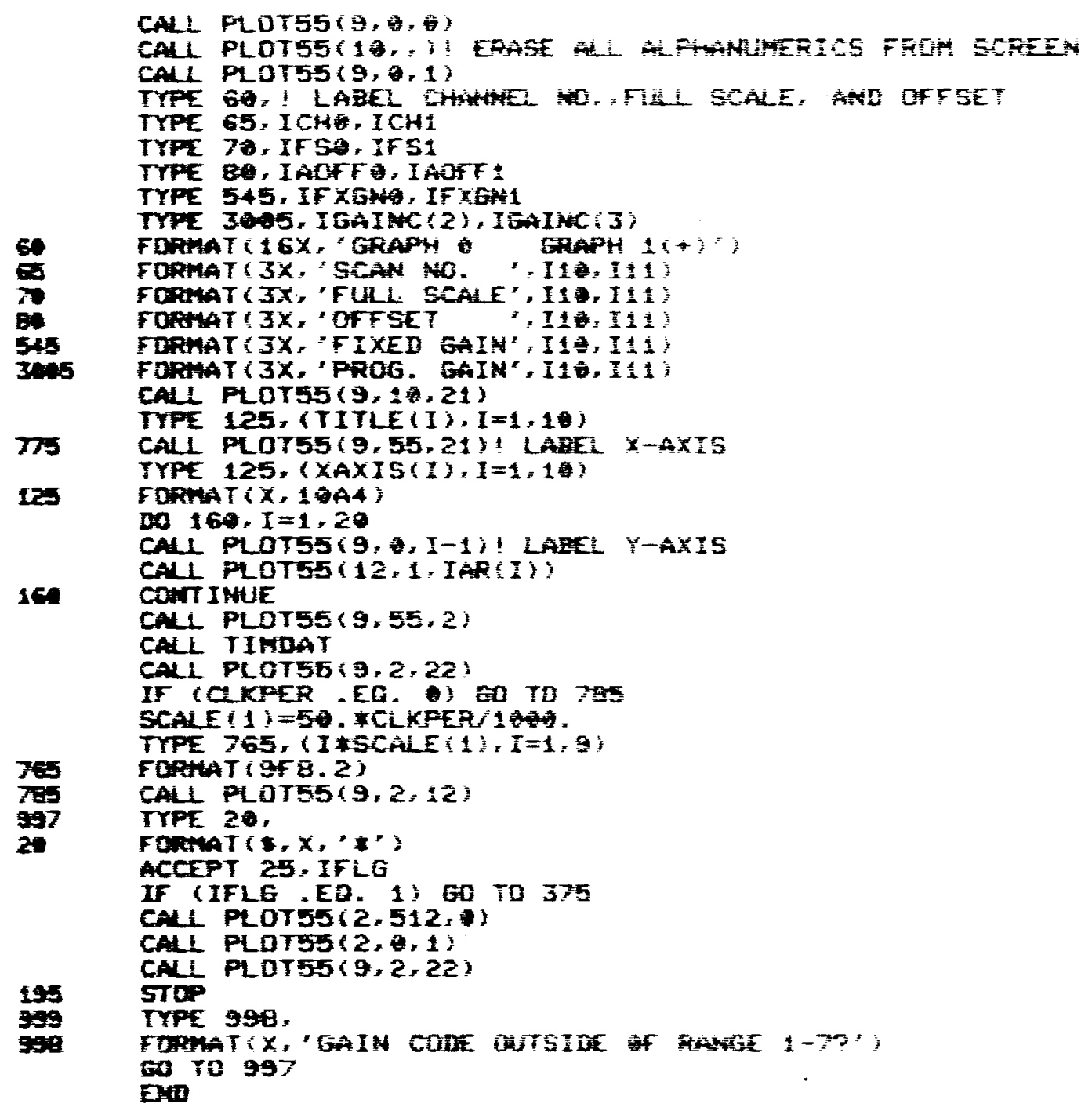

\section{B.3. GRAFAV.FOR LISTING}

TYPE ERAFAV F FR

$c$
$c$
$c$
$c$

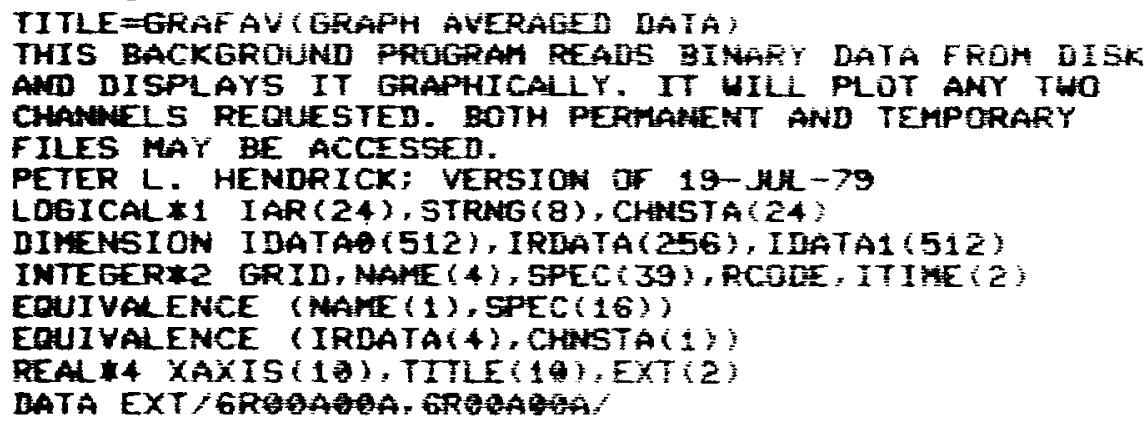


IF (IOSET (5). NE. O) STOP'NOT ENOUGH GUELE SPACE' ICHAY-ISETC! I IALIOCATE CHANGEI ICHAN

TYPE 3 605.

3.AS FORMAT (X,'ENTER 1 TO GRAPH ACTIVE DATA FILE') ACCEPT 125. IIN

IF (IIM NE 1 ) EO $\mathrm{rO}$ toe

IF (ICHCPY (ICHAN, 1).EQ.G) GO TO 379

IF (ICHCPY (ICHAN, S).ED.

TYPE 3410.

3 ist FORMAT $(X$, ' 10 ACTIVE FILE?')

TrPE 390

FOPMAT $(X$, 'ENTER PERMANEMT FILE MANE' $d$ )

IF IICSI (SPEC. EXT, , O) . HE. ? GO TO 40

IF (LDOKLP (ICHAN, NATE) . GE. B) EO TO 370

IF (LOOKLP (ICMAN, NAMI) . NE. -2 ) Go To 1125

37. TYPE 165

10 FORMATIX, 'ENTER STARTING BLOCK MLMER'?

ACOEPT 116, IBLXI

11 Fonitat (I6)

Tret 120 ,

12 FOPHAT $X$, 'ENTER MUHBER OF CHAMUELSISCAN'

ACCEPT 125 . ICNLS

175 FORHAT (I5)

ISTART $=($ ICNA $5+1) / 2+4$

TLETT $=257-I 5 T$ ART

ITIRES= ILEFT/ICNLS

IRC CK5 $=512$, ITIHES

TYPE 1250, ISTART, ILEFT, IERCKS

125

FORMAT ( 315 )

TYPE 20 .

2 FORHAT $(X$, 'ENTER SCAN HLMBERS FOP GPAPHS O AND :" ACCEPT $\$ 615$, ICHA, ICHA

415 FOPHAT $(16, /, 16)$

TYPE 5 e.

5 FGAHTYX, 'ENTER SPAN, GFFET, AND FIXED GAIH FOR GRAPH "; ACCEPT 25, IF 59, I AOFF , IF XONO

TYPE 55.

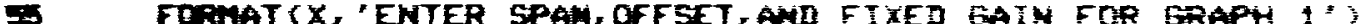
ACCEPT 25, IFS1, IAOFF 1 , IFXEN1

FSe-IF 50

FS1 $=$ IF 51

AOFFA=IAOFF?

ACFF $1=1$ AOFF

FXEMA = IF XENA

$F \times G 41=I F X 5 N 1$

20 FOFmaT(I7, $, 17, \%, 17)$

CA1 PLOT55 (2:1+2+4+32+64+125+266:

Do $915, n=1,512$

IBATAC $(M)=9$

315 IDATAI $(M)=0$

IDK $K=I B K T$

RCOAE = IREAQ (256, IRDATA, IELKI, ISHAN)

If (RCDDE LT. O) 60 ro lood

IF (IUATT (ICHAN) NE. B) GO TO 103

IDATE I IRDATA $(1)$

$\operatorname{ITIRE}(1)=$ IRDATA $(2)$

$\operatorname{ITIR}(2)=\operatorname{IRDA} \operatorname{TA}(3)$

CALL IIMASC ( ITIME, STRAS:

CALL EETGAI (CHASTA (ICHA+1). IPRGH

CALL EETEAT (CMHSTA ( I CH1+1), IPPGNL)

EO 245, $K=1$, IRLCXS

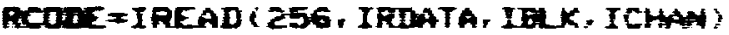

set TELK $=$ KELK $K+1$

If (RCODE EO.-1) GO IO 1000

IF (RCONE LT. $\theta$; GO TO 1090

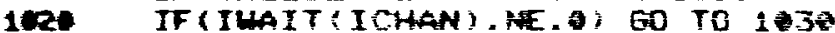




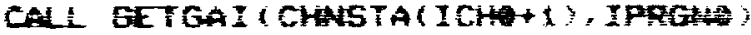

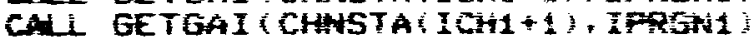

DO $115, j=1$, TTIMES

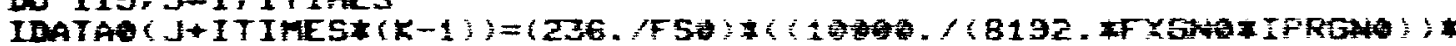

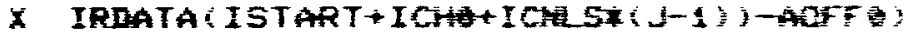

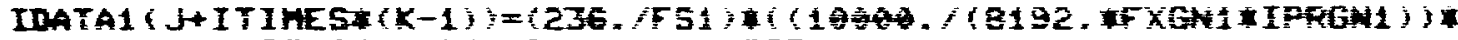

$2 \pm 5$

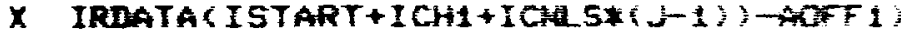

245

CBNTINAE

TINUE

CALL PLDT55(7, , a)

CALI PLOT5S (1.,

CALL PLOTS5 (3, -519, IIATT,

CALL PLDTSS (1, 2, )

CALL PLOTSE 3,516, IDATA1)

13

10 $205,1=1,512,32 !$ PLACE VERTICHL HAKEPS ON GRAPH 1

CAL PLOTEST6, 1,1

CALL PLOT55(4,1,6) I PLACE MORIZOATAL AXIS AT Y= AND 235

TYPE 190.

ACCEPT 210 - GRID

TYPE 230, GRID

FOFNAT $\{X, 14)$

IF CERID . EO. 13 EO TO 32\%

1 1อ.

DO 180, I=1,236,59 IPLACE HORTZONTAL SRITS

Z:.

CALL PLOTS5:4.1.1-1)

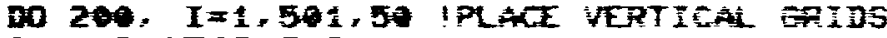

15

210

CALL PLOT5545, T-2, I

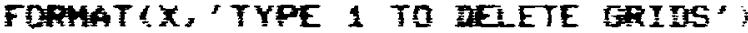

FORTAT $(Y+)$

CONTIHLE

CALI PLOTS54 4, 1, 235)

CALL PLOT55

CALI PLOTS565.5ii.is

TrPE 179 .

ACCEPT 225. (TITLE (IY,I=1,13)

17.

FRAMATCX, "TYPE = SPACE, TITLE DF ERAPH" "

TYPE 220,

ACCEPT $225,(X A X I S(I), I=1,10)$

20.

TrPe 140 .

ACCEPT $150,(I A R(I), I=1,20)$

CALI PLOTSS (9.6. B)

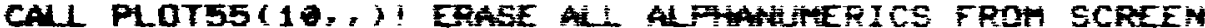

CAL PLOTSS (9,0,1)

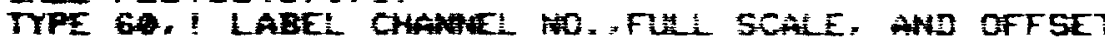

IYPE 55. ICHO. ICHL

TYPE 70, IFSO. IF 51

TYPE 80, IAOFF , IAOFF 1

TYPE 930 , IFXGAG, IF XGN

TIPE 1010. IPRGNS. IPRGNi

Fopiat ( $16 x$, 'GRAPH ह

Framat $3 x$, , SCAN 10 .

ERAPH $i t+3$

FERHT (3X,"OFFSET "I16.I11)

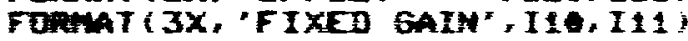

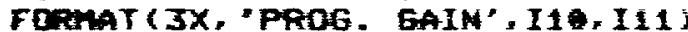

CALL PLOT5S $(9,55,1)$

CALL TIHDAT

Call PLOT55 (9,55,1)

TrPE se7s. IBKKI

57

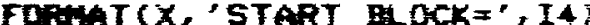

CALL PLDT55 (9,55, 2)

Trpe 4075.

475

FORHAT $C X$, START EATE $=$ "

CALL PLDTS5 (9,66.3)

CALL DECRAT (IDATE)

CAl PLOT55 (9,55,3)

TrPe 925, 15 TRAG $(1), 1=1,5)$ 


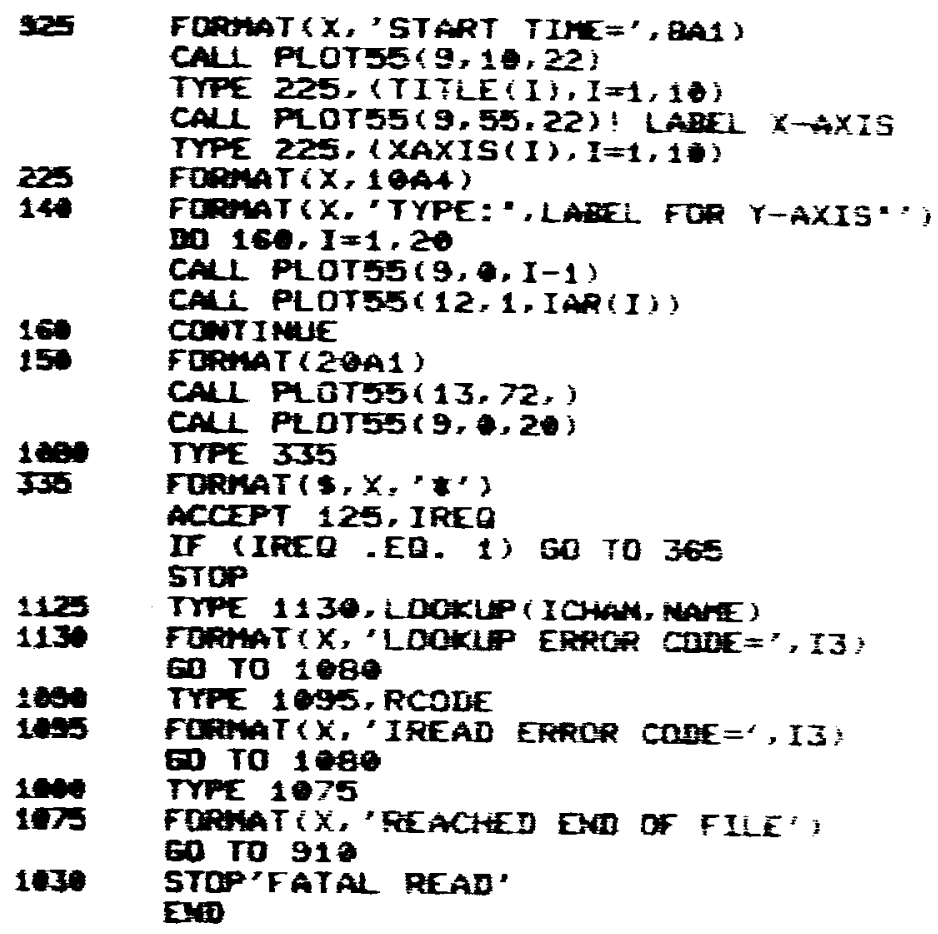

\section{B.4. TYPAV.FOR LISTING}

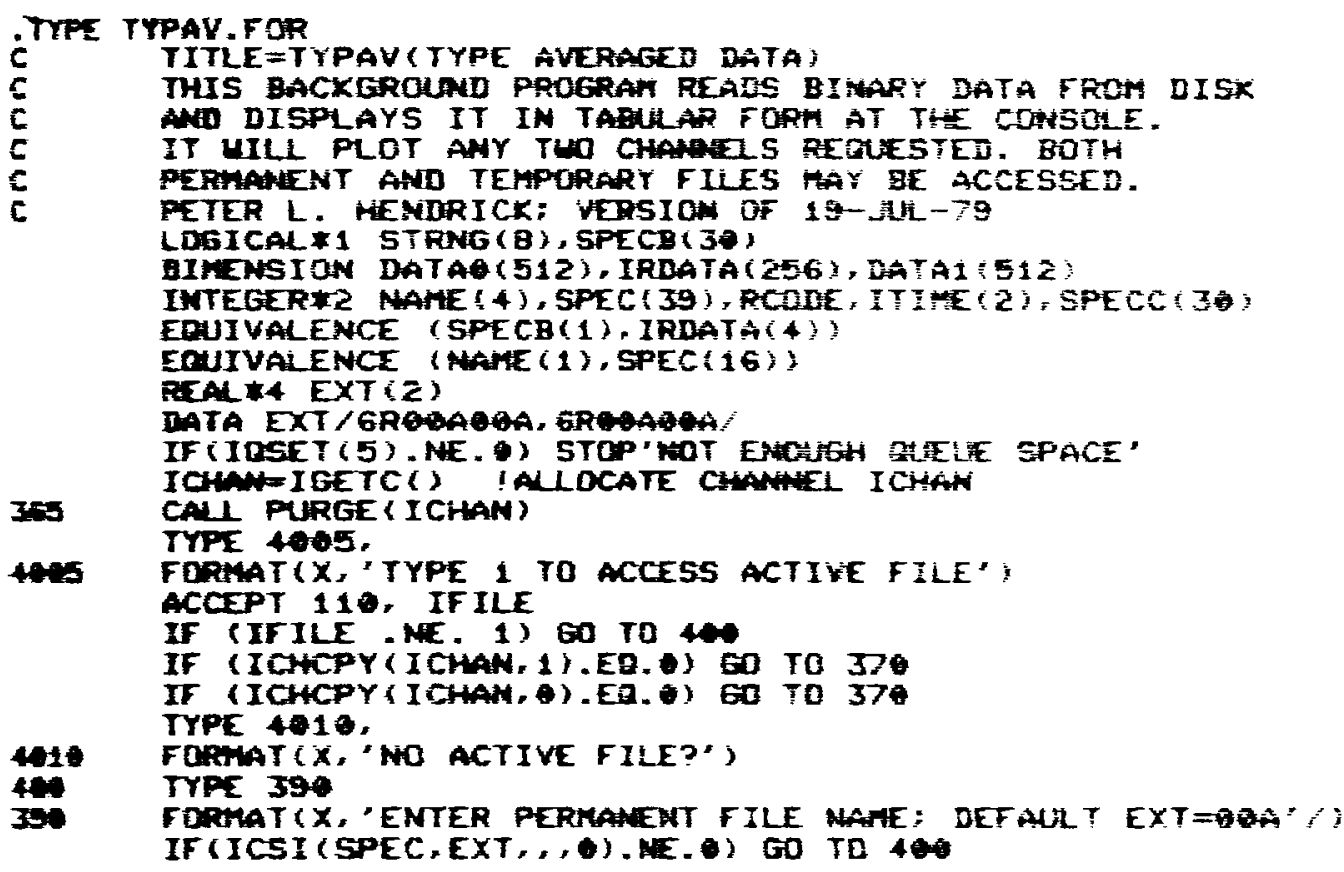




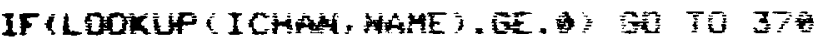

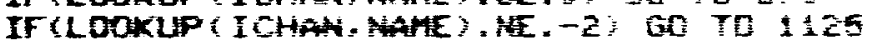

TYPE 175

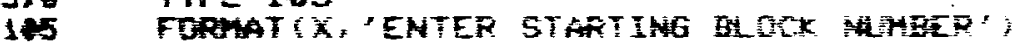

ACCEPT 110 : IELCI

11 FCPMAT is

RCDEE = IREAD / 256, IADATA, I3LXI, ICHAH

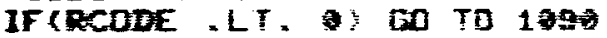

IF (IUAIT I I FHAN) . PE.

ITIHE $12=$ IRDATA $(2)$

ITIRE (Z)=IRDATA I3)

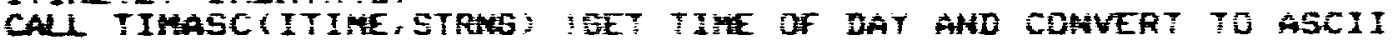

TMPE 120

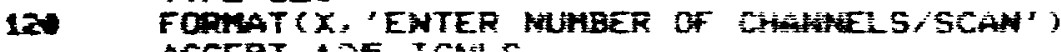

ACCEPT 125. ICALS

125 Format $(15)$

ISTART $=($ ICNLS+1)/2+4

IREPAV $=(257-I S T A R T) / I C H E S$

IELCKS $=512 / 1$ PEPAV

TrPe 2000 .

20

FOPHAT $X$, "EMTER 1 TO TYPE FIRST BLOTK SPEEIFIED";

2015

ACCEPT 2005.1 REDST

FDRTAT ( IG)

IF (TRERST - NE. 1) 60 T0 3Gas

2415

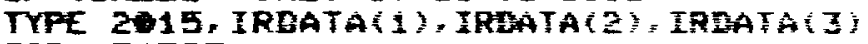

FOMUAT $(307$ )

Do 4015, 51 , ICNLS

SPECC $(j)=\operatorname{SPE} C \mathrm{~B}(\mathrm{j})$

IF (SPECC $(j)$. SE. Q) 50 $10+315$

$\operatorname{sitcc}(j)=\operatorname{spECC}(j)+256$

4015 CONTINUE

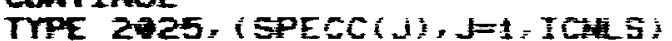

zas

FERHAT $X, 1206$;

2940 TYPE 2040

FORHAT (X)

TYPE 2430, (IRDATA

2030

3705

Fanthi $(x, 1216)$

TrPe 2059 .

FORHATKX, ${ }^{x}$ ENTER 1 TO TYPE 2 OHAHELS IN HILLIVCLTS"y ACCEPT 25, IREQS

IT IRERS. NE. I) 60 T0 1000

TYPE 20,

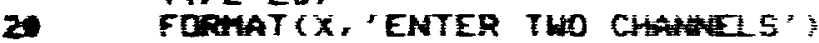

ACCEPT 9es. I CHA. ICH!

Ses FORHATIS., I6)

TYPE 50 .

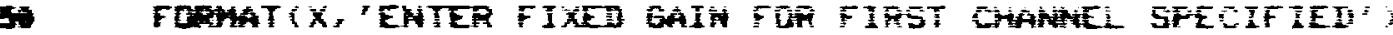
ACCEPT 25. IFXENA

THPE 5\$,

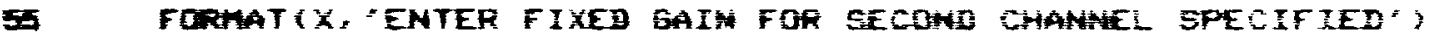
ACCEPT 25. IF XGN1

FXENOIFXONO

FXFNL = IFXEN

PAEMA IFRGH

PREML $=$ IPRGMS

23 FORAT (17)

Do $915, H=1.512$

Batae $(M)=0$

Its EMTA $(M)=0$

IDUK=IBUKI

TrPe 4675.

475 FOANAT $(\$, x, "$ "START DATE $=$ "

CALL DECRAT I IRDATAC1 i)

TrPE 925, (5TANG (1), $I=1,3$ )

nas FERAT $X$, 'START TIHI', EAI'

TYPE 1025.

4has Fonm 


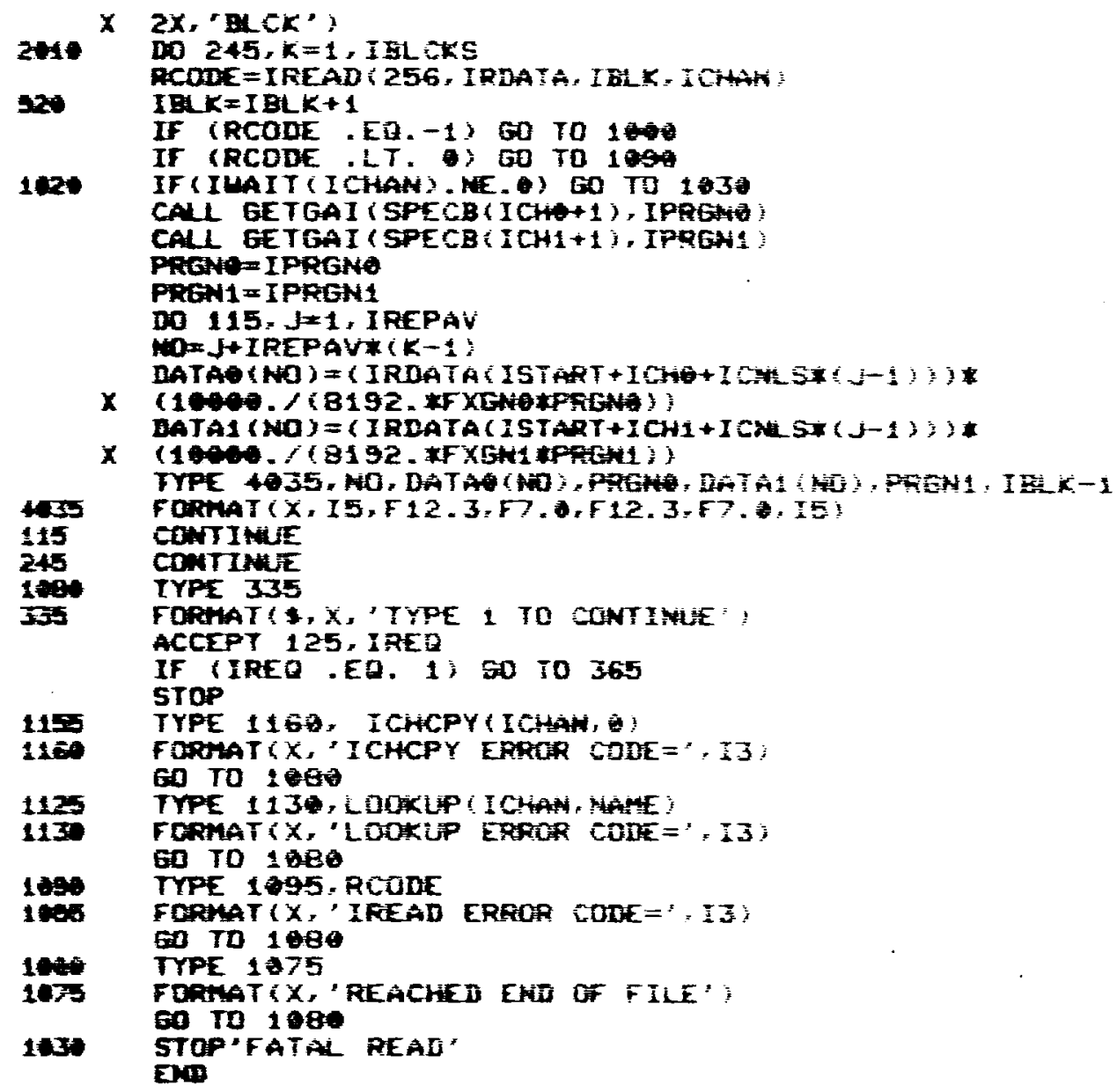

\section{B.5 TYPLOG.FOR LISTING}

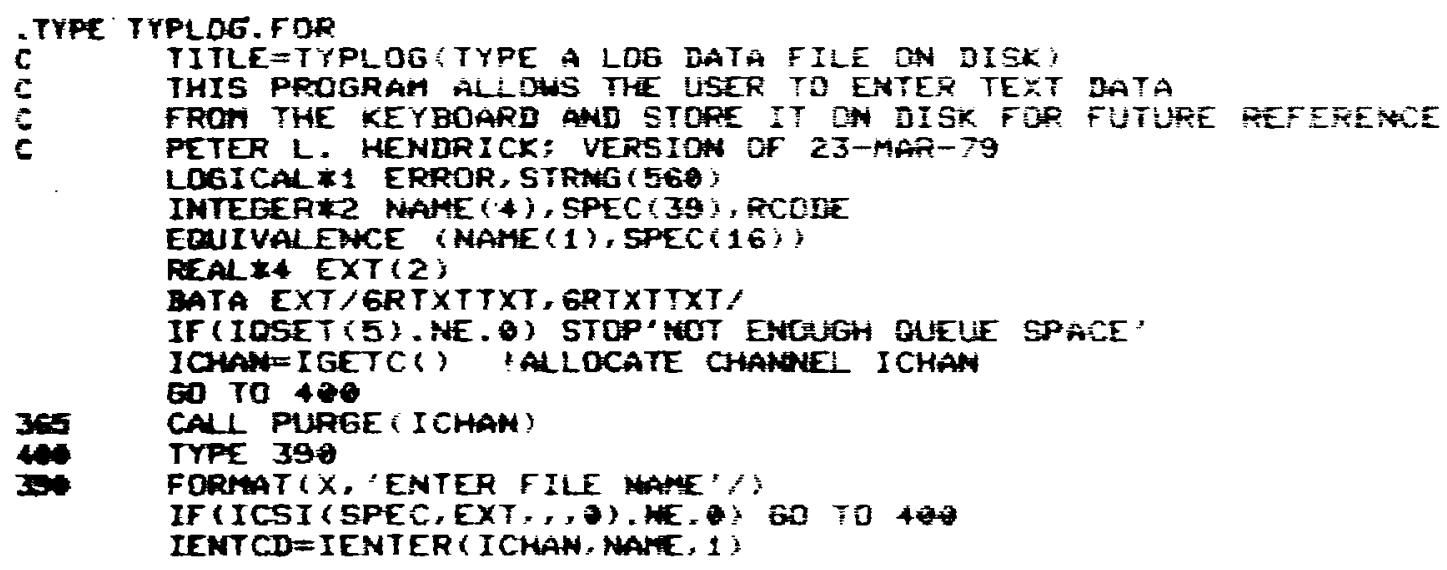


IF (IENTCD . ED. -1) GO TO 2 TES

IF (IENTCD. LT. \$) OD TS ETis

TYPE 2005.

2005 FORMATIX, 'ENTER ELINES OF TEXT DATA'

$103020, L=1,6$

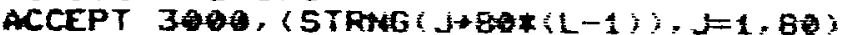

FORMAT ( BOA 1 )

CONT INUE

$\operatorname{STRan}(481)=\theta$

HACONE = IURT TH $(256,5 T \mathrm{THE}, 0,6)$

IF (WRCOBE IT. O GO TO 2445

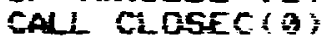

15Fe TYPE 335

339 FORATIX, "TYPE 1 TO TYPE HORE DATA"

125 FOTHAT $15 \overline{1}$

IF (IAEQ EQ. 1) 00 TO 365

STOP

2015

29:

TYPE 2020 , TENTCD

$2 \cos$

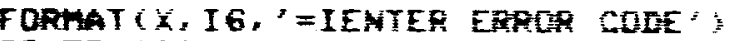

50 To 1950

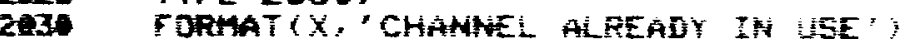

50 TO 1680

2045 TTPE 2050. WRCODE

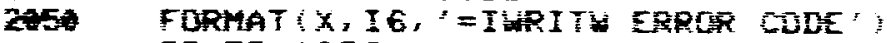

30 T0 1080

EMD

\section{B. 6 RETXT.FOR LISTING}

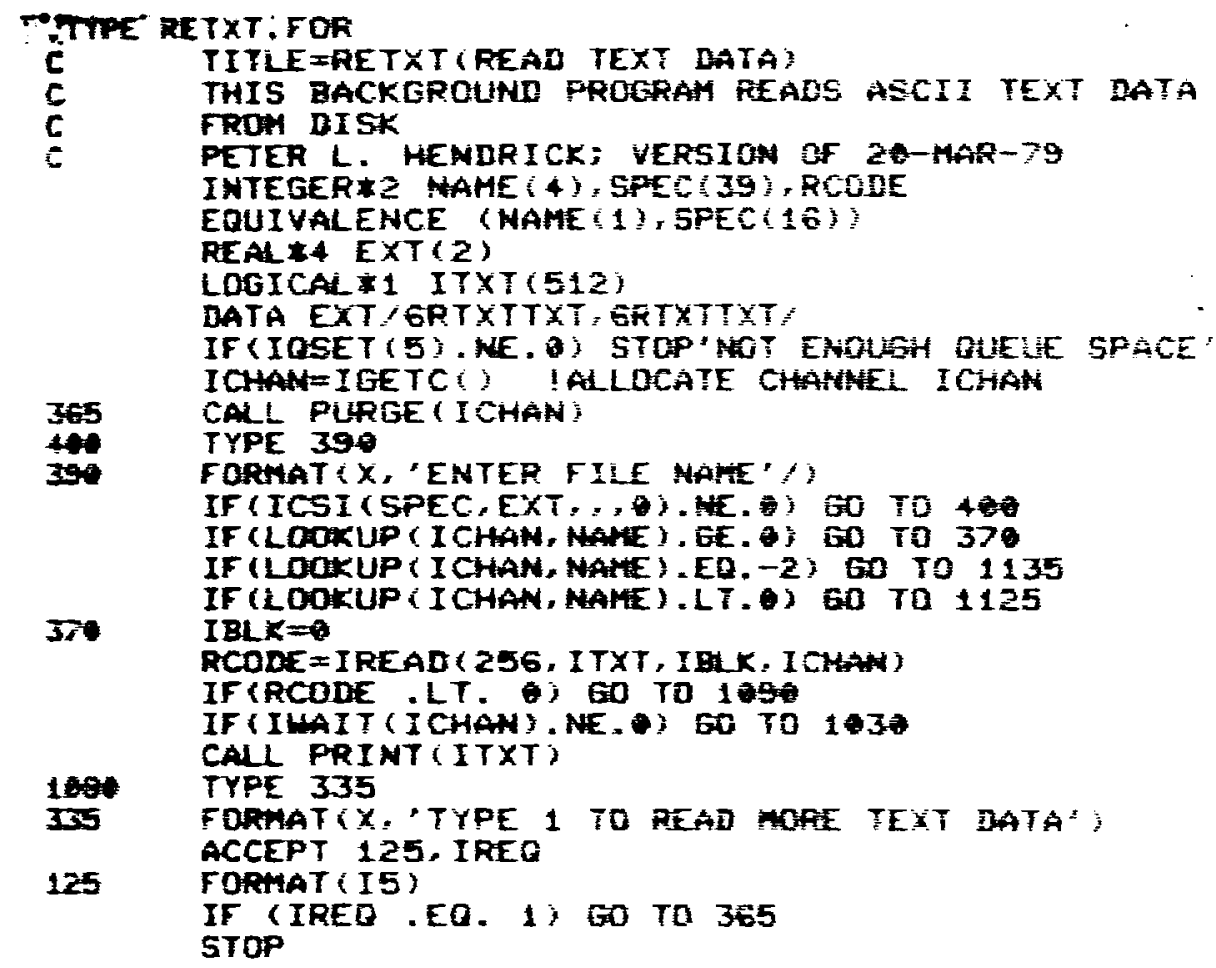




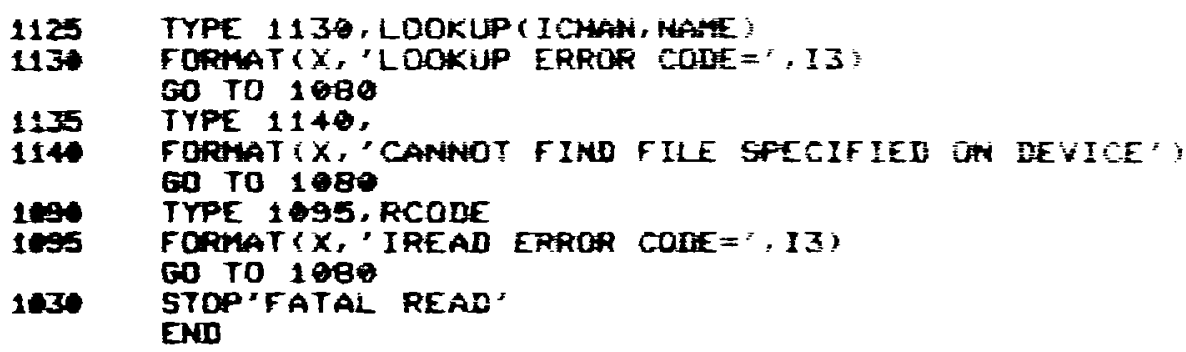

\section{B.7 REINT3.FOR LISTING}

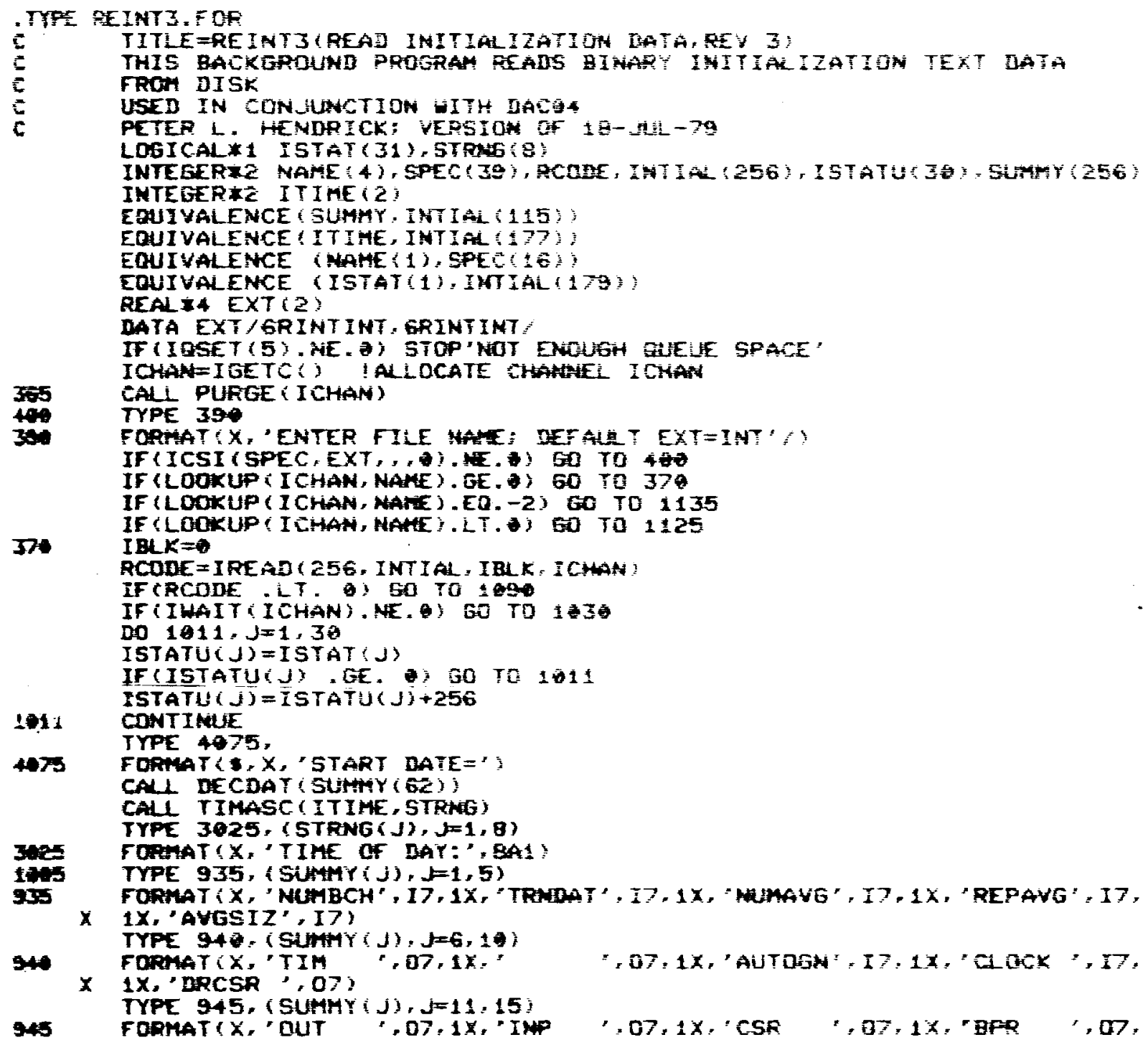


$x \quad 1 x$, 'DgouT 1',07)

90

$1 X, 7001$ TYPE 3820 ,

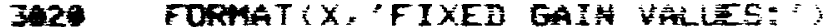

x+10

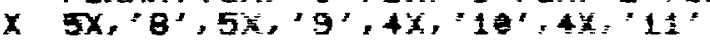

TYPE 3edes, (Suthy ( $j), j=18.29$

Fete FoRAt $(x, 1216$

TYPE Je15.

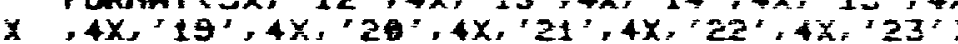

TrPe 3005 , (SUMHY $(J), J=30,41$ )

TYPE 4005.

4as

419

TYPE 4e19.

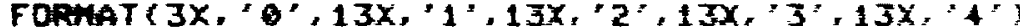

TYPE 4e15, (SUAHY (J), $=42,51$ )

415

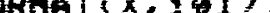

TYPE 4020.

43

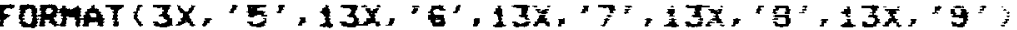

TTPE 4015, (SUAHY $(J), j=52,61)$

TYPE 355

g도

390 TYPE 965 .

seso

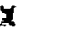

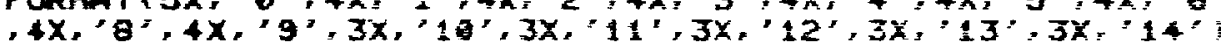

TYPE 960: (ISTAFU J), $j=1,15$ )

FoRAAT ( $X, 1505$ )

390

57

FORKAT $X$ )

TTPE 970 ,

$\mathbf{x}$

$x+32^{\prime}, 3 x, 3,23$

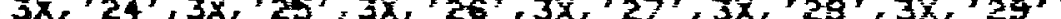

TYPE 969, iISTATU $J\}, j=16,30$ ?

LWI TYPE $\mathbf{3 3 5}$

335 FORMAT $X{ }^{\prime}$ 'TYPE 1 TO REAE MORE IHITIALIZATION BATA" ACCEPT 125, IREQ

125 FORTAT $(15)$

If (IREQ .EQ. 1) GO TO 365

STOP

$1+25$

113

$11 \overline{30}$

U4t

109

1095

1030

TTPE 1136. LDOKUP I INAN, HAYE

FORMAT $X$, , LCCKUP ERROR COTE $=*$, 3 ;

EO TO 1680

TYPE 1140 .

FORMAT $(X$, 'CAMNOT FIND FIIE SPECIFIES DN WEYICE",

50 To 1 क80

TYPE 1095. RCONE

FORHAT $X X$, IPEAD ERROA CODE=", I3

GO TO 1 B8?

STUP FATAL READ

EMD

\section{B. 8 RESET.FOR LISTING}

CALL PLOTSS (2,512,

5 TOP

END 


\section{B.9 GETGAI.MAC LISTING}

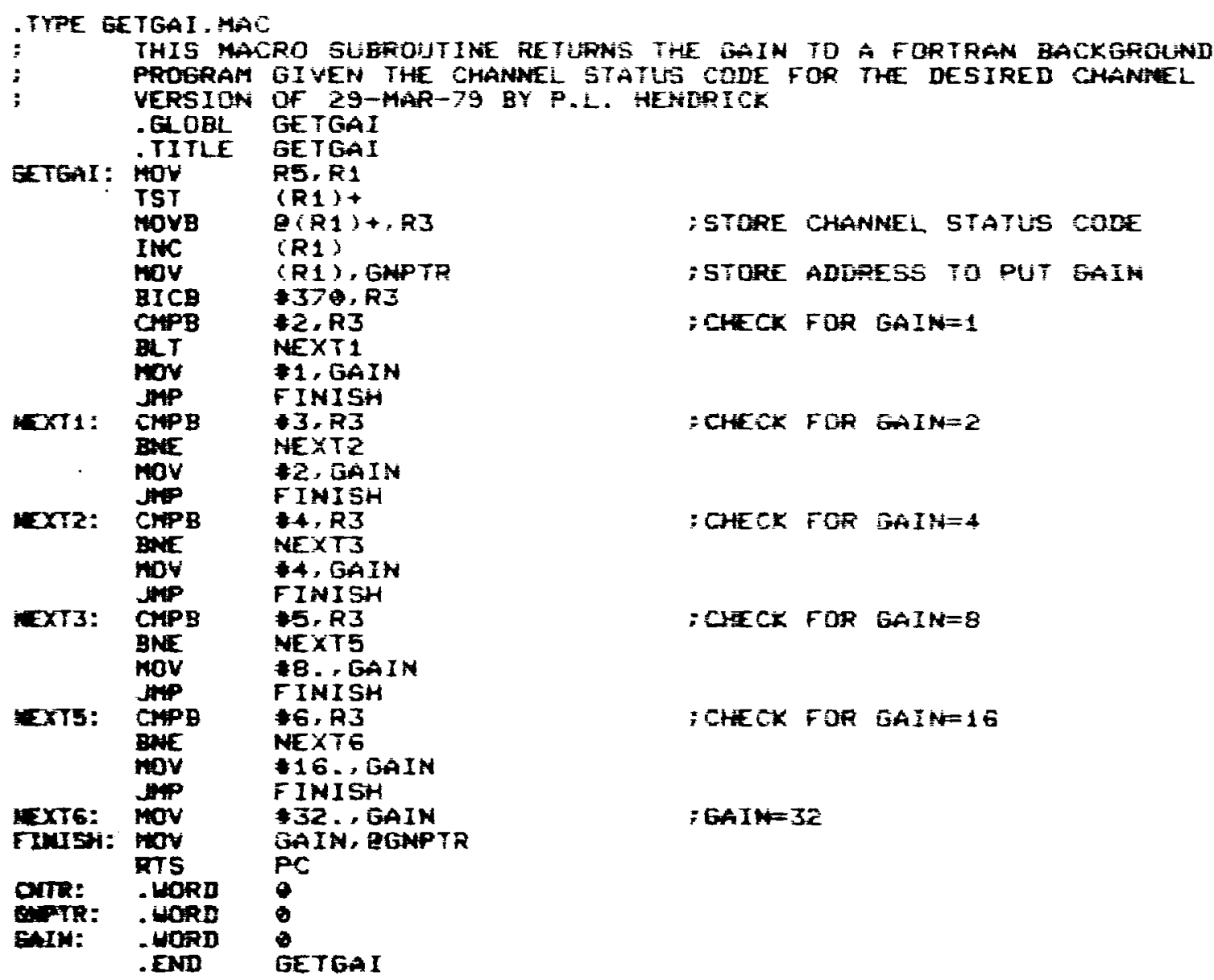

B. 10 TIMDAT.MAC LISTING

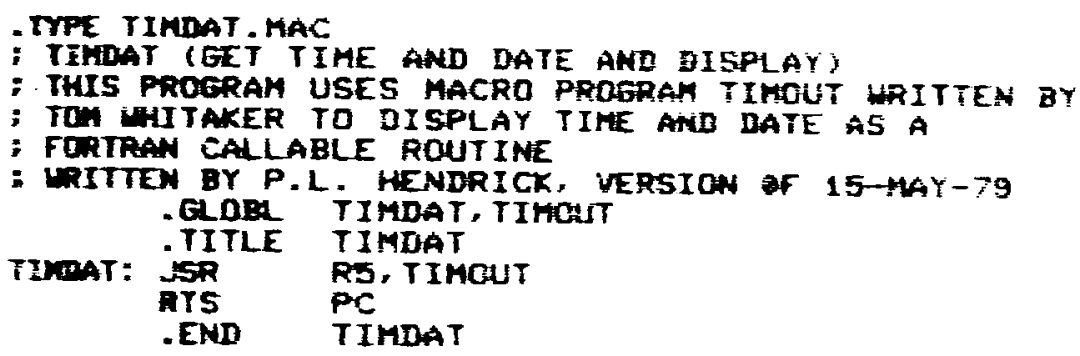




\section{B.11 DECDAT.MAC LISTING}

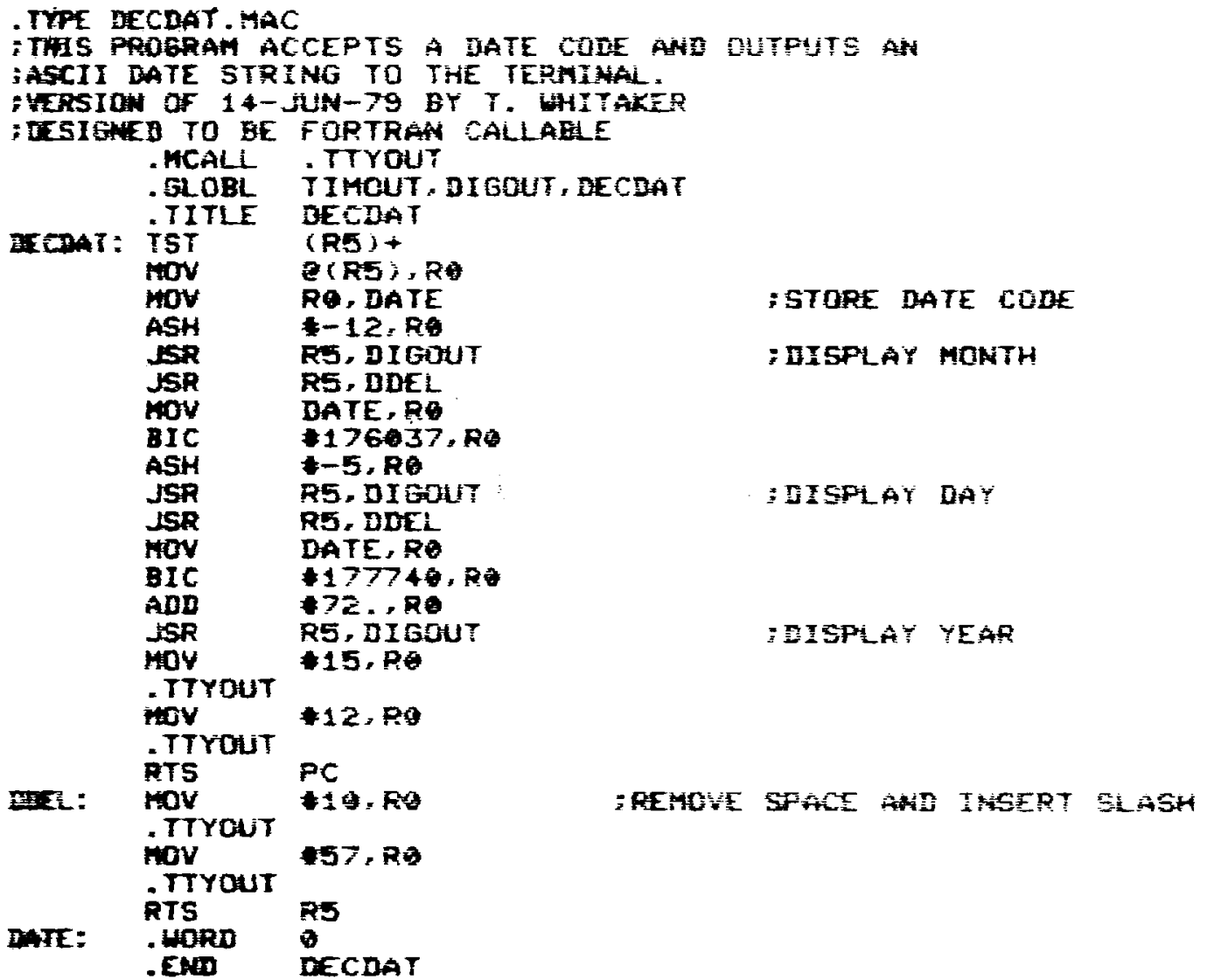

IATE: 


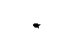

- 


\section{APPENDIX C}

PROCEDURE FOR MAKING DELETED FILES PERMANENT 


\section{APPENDIX C}

\section{PROCEDURE FOR MAKING DELETED FILES PERMANENT}

In certain cases (e.g., a system crash) the user may need to make a file permanent that was previously deleted. For example, should the system crash while DACØ4 is operating, the current data file will not be made permanent (i.e., the directory will not list the file name). Since the data will remain intact until that area of the disk is rewritten, it is possible to modify the directory to cause the data file to become permanent. The following procedure will make a deleted file permanent:

1. Reboot the system with system disk in drive 0 and deleted file in drive 1.

2. Obtain a directory of the deleted file. for example:

.DIR/DEL/BLO[CR]

DATA. $\emptyset \mathrm{A}$

$480 \quad 14$

where, in this example, the deleted file, DATA.ØØA, started at absolute block 14 and had 480 blocks orginally allocated to it.

3. Convert the decimal numbers above (i.e., starting block number and temporary length) to octal.

4. Call DUP:

.R DUP [CR]

$\star D X]: D A T A . \emptyset \emptyset A=/ C: 16: 74 \emptyset$ [CR]

where $16_{8}=14_{10}$ and $740_{8}=4_{10}$

5. Run DIR to confirm results. Data file DATA. 96 A, should now be listed as a permanent file. 


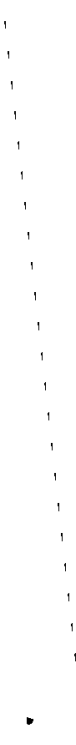


APPENDIX D

HARDWARE ADDRESS AND VECTOR LOCATIONS 
APPENDIX D

HARDWARE ADDRESS AND VECTOR LOCATIONS

A11 hardware interfaces are addressed in a manner identical to memory in the PDPIIV/03. Generally the address space between $28 \mathrm{~K}$ and $32 \mathrm{~K}$ is reserved for I/O addresses, thus leaving 0-28K available for memory. Some I/O addresses have been altered, however, to allow an effective memory space of $29 \mathrm{~K}$. The current addresses are summarized in Table D.1. If the addresses for the ROM bootstrap and DRVII parallel interfaces were relocated to a higher address, an effective memory of $30 \mathrm{~K}$ could be realized. In the case of the DRVIl paraliel interface this is easily accomplished. However, a method has not been discovered to relocate the ROM bootstrap addresses.

Many of the interfaces listed in Table D.l can be operated on an interrupt basis if desired. In the present application, only the real-time programmable clock utilizes the interrupt feature. The clock interrupt vector is located at octal 440. Most installed interface cards have DIP switches, which allow either the interface address or interrupt vector addresses to be altered within a given range. 
IABLE D.1. Summary of I/0 Addresses

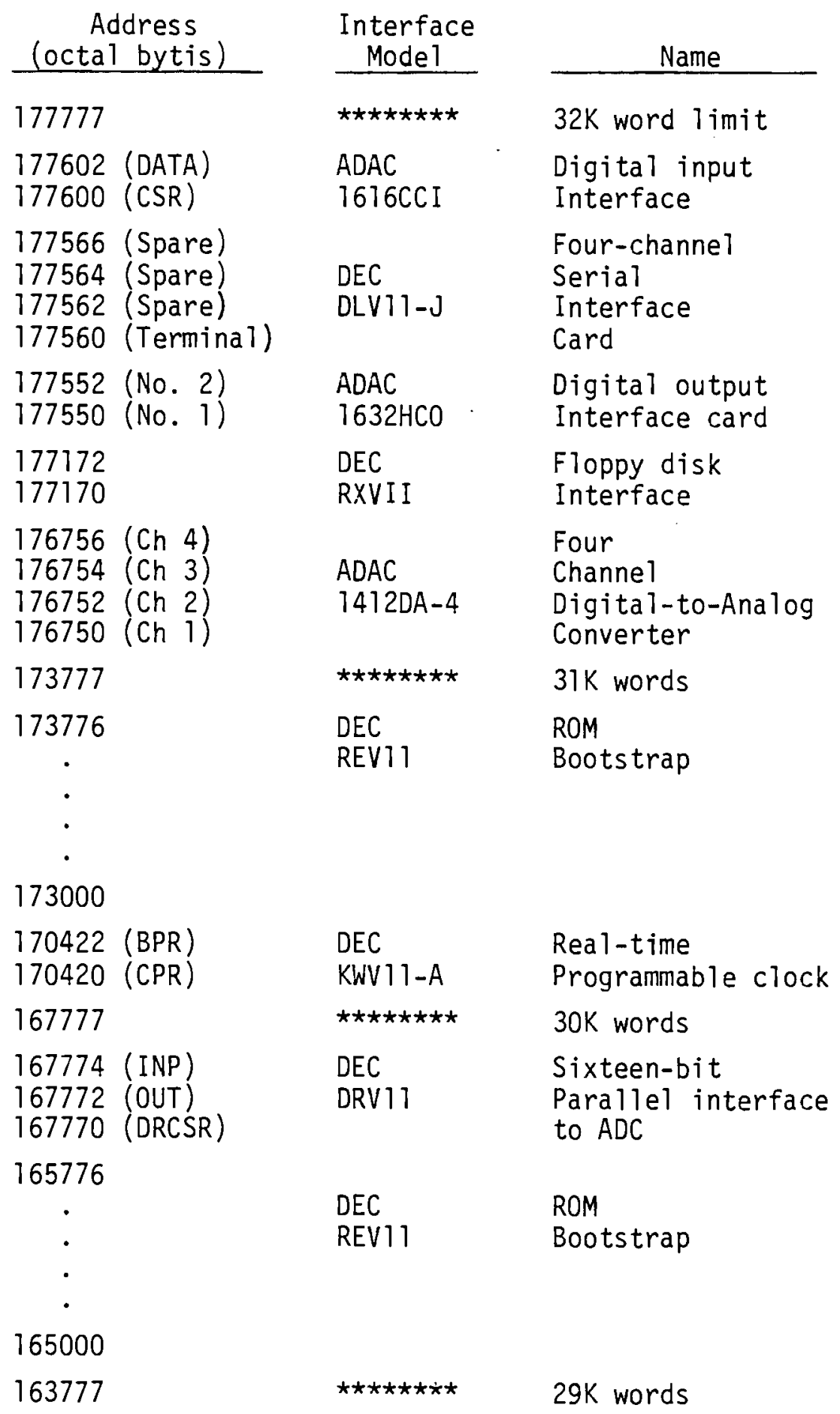


APPENDIX E

SPECIAL TIMING CONSIDERATIONS FOR DACD4 
APPENDIX E

SPECIAL TIMING CONSIDERATIONS FOR DACØ4

The number of channels scanned, the number of data values averaged and the clock interrupt period together dictate the overhead on the computer (i.e., CPU). The user must insure that a 11 clock interrupts are serviced, all writes to disk are performed, and that ample time remains to perform desired jobs in background.

To permit sufficient time for disk writes, it is recommended that the period between disk writes be set to 1 min or longer. This period, T, can be calculated as follows:

$$
T=t A S
$$

where: $T=$ disk write period in seconds

$t=$ clock interrupt period in seconds

$A=$ number of words averaged

$S=$ number of complete scans per disk block as calculated in Section 5.1 and shown in Table 6 .

For example, a clock interrupt period of $10 \mathrm{msec}$, with 600 averages taken and 24 channels scanned, would give a 1-min disk write interval. In special cases, periods as short as $15 \mathrm{sec}$ have been utilized. However, all background operations anticipated should be tested under these conditions before committing to a test.

A second criterion on the operation of DAC $\emptyset 4$ relates to the CPU overhead. It is evident that the more channels scanned the longer the program spends in the clock interrupt service routine which, therefore, increases the computer overhead. Therefore, for each particular number of channels scanned there is a clock interrupt period below which DAC $\emptyset 4$ will not allow background programs. to run. Figure E. 1 shows test data obtained during development for a disk write period of $20 \mathrm{sec}$. Based upon these data, a clock interrupt period equal to or greater than $10 \mathrm{msec}$ is recommended. In special cases the graph may serve as a guide for the selection of a shorter period; however, the proper operation of DACØ4 should be verified before committing to a test. 


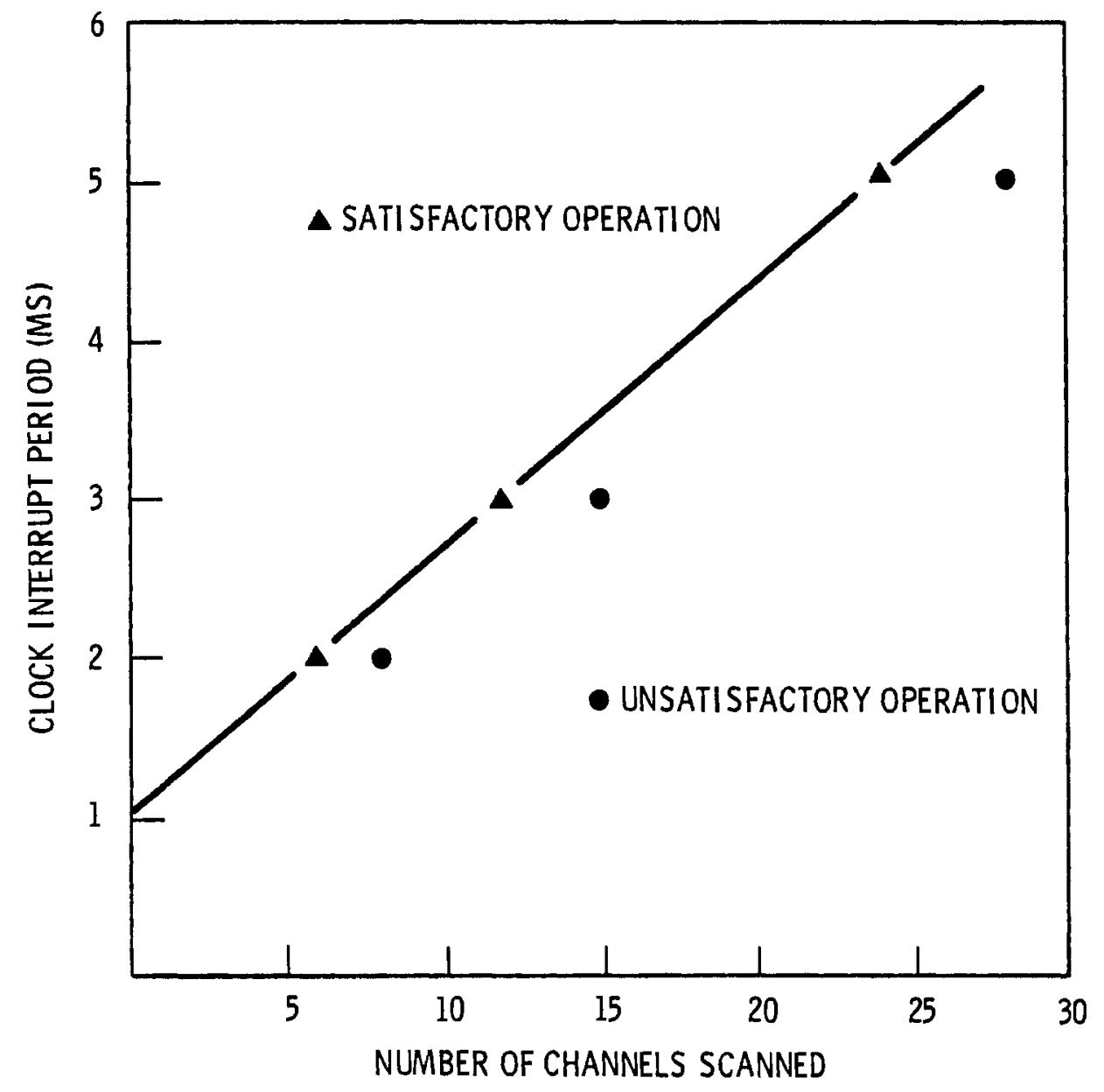

FIGURE E.1 DACD4 Operating Regimes 


\section{REFERENCES}

1. RT-11 V3B Advanced Programmer's Guide, Digital Equipment Corp., Maynard, MA, JuTy 1978.

2. RT-11 V3B System User's Guide, Digital Equipment Corp., Maynard, MA, A.ugust 1977.

3. RT-11 V3B System Generation Manual, Digital Equipment Corp., Maynard, MA, March 1978.

4. System 620 Operation and Maintenance Manual, Series 100/200 Amplifier/ Multiplexer, Neff Instrument Corp., Duarte, CA, August 1977.

5. Memories and Peripherals Handbook, Digital Equipment Corp., Maynard, MA, 1978-1979.

6. Model $1632 \mathrm{HCO}, 16$ and 32 Bit High Current Output Interface Instruction Manual, ADAC Corp., Woburn, MA, August 1977.

7. Model 1616 CCI, 16 Line Contact Closure Module Instruction Manual, ADAC Corp., Woburn, MA, February 1978.

8. Instruction Manual for ADAC Corp, Mode1 1412 DA Data Acquisition and Control Sys tem, ADAC Corp., Woburn, MA, September 1978. 


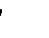




\section{DISTRIBUTION}

No. of

Copies

OFFSITE

1 DOE Chicago Patent Group 9800 South Cass Avenue

Argonne, IL 60439

A. A. Churm

1 DOE Division of Basic Energy Science Washington, D.C. 20545

D. K. Stevens

27 DOE Technical Information Center

3 University of Washington Seattle, WA 98195

D. H. Polonis

R. G. Stang

W. G. Weitkamp

1 T. C. Reilley

Oak Ridge National Laboratory

P.0. Box $X$

Oak Ridge, TN 37830

1 F. V. Nolfi

Argonne National Laboratory 9700 South Cass Ave.

Argonne, IL 60439
No. of

Copies

ONSITE

1 DOE Richland Operations Office

H. E. Ransom

Pacific Northwest Laboratory

E. R. Bradley

J. L. Brimhal1

L. A. Charlot

E. L. Courtright

C. R. Hann

C. H. Henager, Jr. (2)

P. L. Hendrick (15)

J. L. Hooper

R. H. Jones

H. E. Kissinger

R. P. Marshall

R. D. Nelson

N. J. Olson

C. Pavloff

E. P. Simonen

T. J. Whitaker (2)

Technical Information (5)

Publishing Coordination (2)

Goo

$1 \quad$ Hanford Engineering Development Laboratory

J. L. Straalsund 
